\title{
WestVirginiaUniversity
}

THE RESEARCH REPOSITORY @ WVU

Graduate Theses, Dissertations, and Problem Reports

2006

\section{Multi-impression enhancement of fingerprint images}

\author{
Mayank Agarwal \\ West Virginia University
}

Follow this and additional works at: https://researchrepository.wvu.edu/etd

\section{Recommended Citation}

Agarwal, Mayank, "Multi-impression enhancement of fingerprint images" (2006). Graduate Theses, Dissertations, and Problem Reports. 1691.

https://researchrepository.wvu.edu/etd/1691

This Thesis is protected by copyright and/or related rights. It has been brought to you by the The Research Repository @ WVU with permission from the rights-holder(s). You are free to use this Thesis in any way that is permitted by the copyright and related rights legislation that applies to your use. For other uses you must obtain permission from the rights-holder(s) directly, unless additional rights are indicated by a Creative Commons license in the record and/ or on the work itself. This Thesis has been accepted for inclusion in WVU Graduate Theses, Dissertations, and Problem Reports collection by an authorized administrator of The Research Repository @ WVU. For more information, please contact researchrepository@mail.wvu.edu. 


\title{
Multi-Impression Enhancement of Fingerprint Images
}

\author{
by \\ Mayank Agarwal
}

Thesis Submitted to the

College of Engineering and Mineral Resources

at West Virginia University

in partial fulfillment of the requirements

for the degree of

Master of Science

in

Electrical Engineering

Approved by

Dr. Xin Li, Ph.D., Committee Chairperson

Dr. Arun A. Ross, Ph.D.

Dr. Natalia Schmid, Ph.D.

Lane Department of Computer Science and Electrical Engineering

Morgantown, WV

2006

Keywords: Biometrics, Fingerprint, Coherence of Direction, Image Inpainting, Fingerprint Enhancement, Optical Flow.

Copyright 2006 Mayank Agarwal 


\section{Abstract \\ Multi-Impression Enhancement of Fingerprint Images \\ Mayank Agarwal}

Biometric system deals with recognizing a person by determining the authenticity based on physiological and behavioral characteristics. Fingerprints are well known for their distinctiveness among individuals and have been widely used for personal authentication and recognition. This thesis studies the enhancement problem for fingerprint images with marginal quality due to a number of reasons (e.g., scar, moisture, dryness, over-pressure).

Our contribution is two fold. In single-copy enhancement, we have developed an image inpainting based approach to repair low-quality regions in a fingerprint images that are detected by ridge coherence. In multi-copy enhancement, we propose to mosaic multiple copies of the same finger by optical-flow based registration and Least-Square based interpolation. Experimental results are reported to validate the effectiveness of the proposed techniques. Some conclusions about the future study are made. 


\section{ACKNOWLEDGMENTS}

I am eternally grateful to my advisor Dr. Xin Li for his constant support and encouragement. He is the basic reason for the metamorphosis that took place in my ability to contemplate and research. I thank Dr. Li for all the intellectually stimulating conversations and extremely helpful discussions we had.

I thank the members of my committee Dr. Arun Ross and Dr. Natalia Schmid for their patience, guidance and valuable inputs. I also thank all the faculty members of WVU CSEE department for all their help and guidance. I would also like to thank my lab mates at the Image and Video Coding Lab for their support and interactive technical discussions which often gave me ideas for my research.

I thank all my friends in United States and in India for their continuous support and blessings. Finally, I want to thank my parents Mr. and Mrs. Agarwal and my brother Mr. Priyank Agarwal for their support, blessings and encouragement. 


\section{TABLE OF CONTENTS}

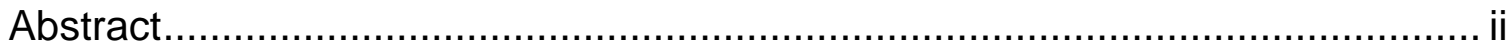

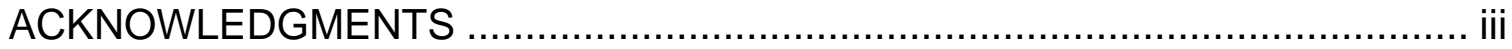

TABLE OF CONTENTS ............................................................................ iv

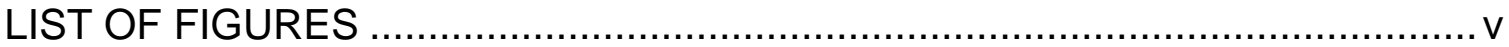

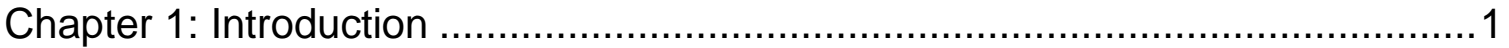

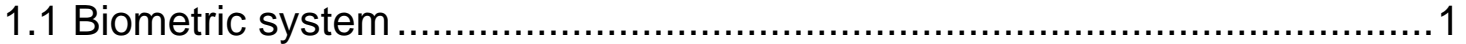

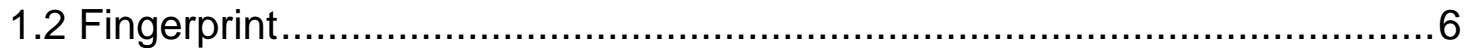

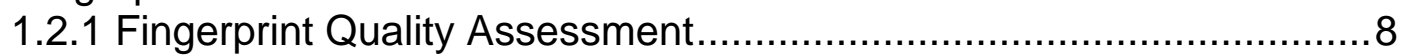

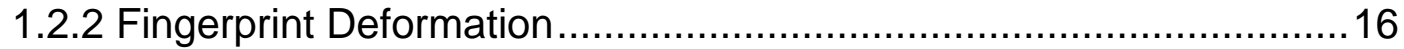

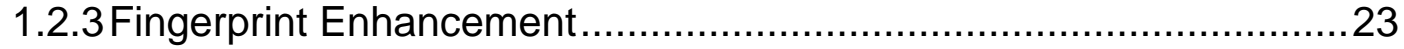

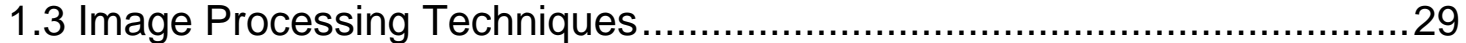

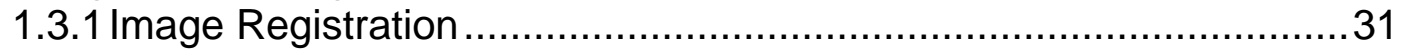

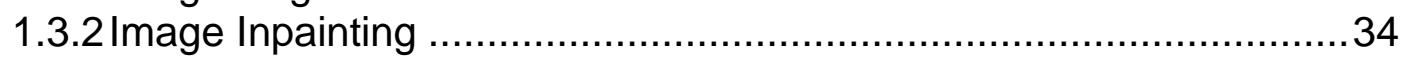

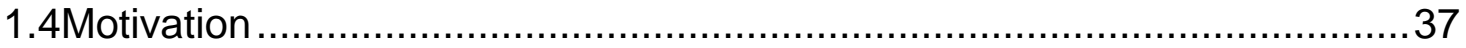

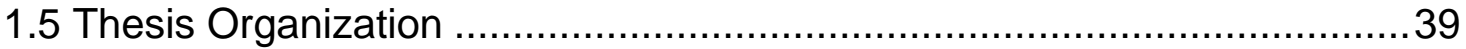

Chapter 2: Fingerprint Quality Assessment ………................................. 41

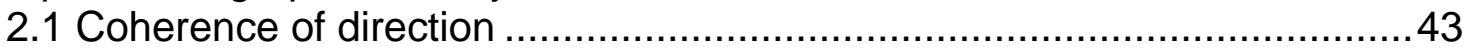

2.2 Low quality region detection .......................................................... 44

Chapter 3: Fingerprint Enhancement: Single Impression.............................. 48

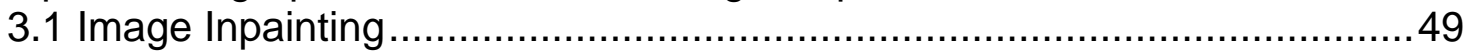

3.1.1 Orientation Adaptive Interpolation ............................................ 51

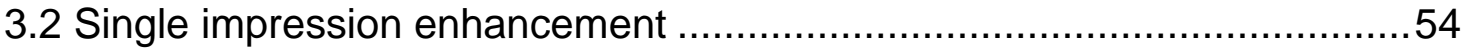

Chapter 4: Fingerprint Enhancement: Multiple Impression .............................56

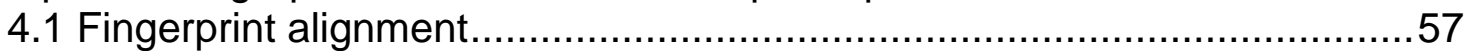

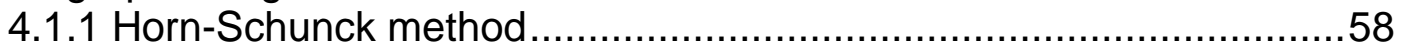

4.2 Multi-impression fingerprint enhancement ....................................65

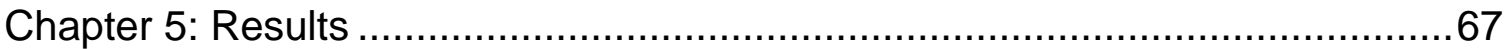

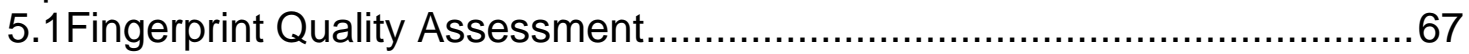

5.2Single Impression Fingerprint Enhancement...................................... 70

5.3Multi-Impression Fingerprint Enhancement ....................................... 74

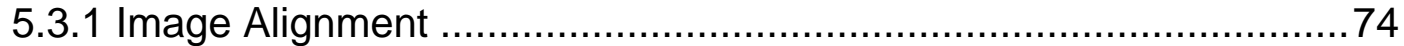

5.3.3 Binary-level fingerprint image enhancement .................................79

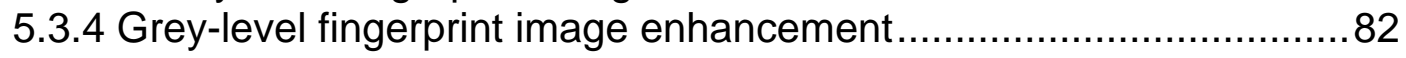

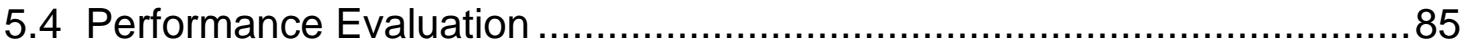

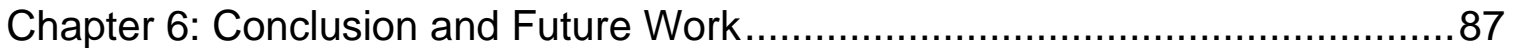

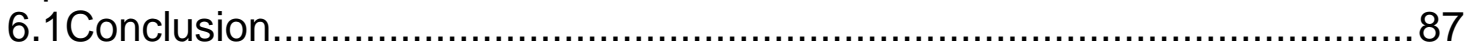

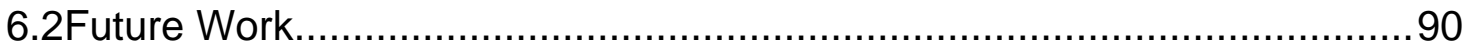

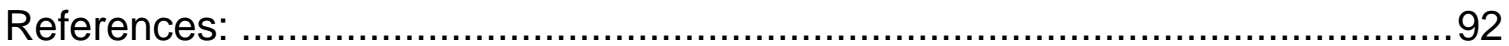




\section{LIST OF FIGURES}

Figure 1.1: High level schematic of a generic biometric system........................

Figure 1.2: Verification mode....................................................

Figure 1.3: Identification mode..................................................

Figure 1.4: Features of a fingerprint........................................... 6

Figure 1.5: (a) Good quality image, (b) Fingerprint with dry areas, (c) Fingerprint with scars, (d) Fingerprint with wet areas............................. 9

Figure 1.6: A typical texture-like ridge block................................. 12

Figure 1.7: Fingerprint images with their power spectrum.........................15

Figure 1.8: Alignment of two impressions of same finger after affine transform........17

Figure 1.9: (a) Original impression, (b) Average deformation model on a

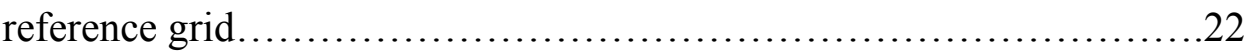

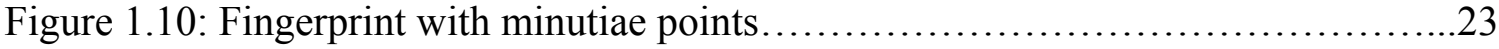

Figure 1.11: (a) Input fingerprint image, (b) Enhanced fingerprint image................29

Figure 1.12: (a) Original image, (b) Object in the foreground is removed and the region is filled, (c)"Lena" image degraded with artificial artifacts, (d) Restored "Lena"............................................................35

Figure 2.1: Fingerprint image (a) with dry areas, (b) with scars, (c) wet areas...........42

Figure 2.2: Gradients in part of a fingerprint..................................43

Figure 2.3: The averaged directional field in part of a fingerprint $\ldots \ldots \ldots \ldots \ldots \ldots \ldots . .44$

Figure 2.4: (a) Original fingerprint image, (b) Variance field of the fingerprint image,

(c) Segmented fingerprint image.................................45

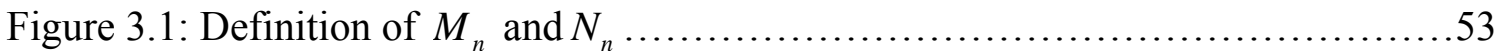


Figure 3.2: (a) Original fingerprint image with wet areas; (b) Binary mask with low quality regions as $0 ;(\mathrm{c})$ Fingerprint image with masked low quality

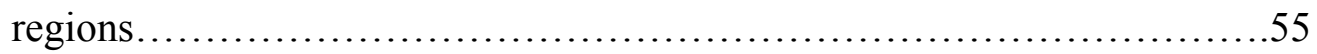

Figure 4.1: The image at position $(x, y, t)$ is same as at $(x+\delta x, y+\delta y, t+\delta t) \ldots \ldots \ldots . . .59$

Figure 4.2: (a) The aperture problem: only normal velocity $\vec{v}_{n}$ can be recovered but tangential velocity $\vec{v}_{t}$ cannot; (b) The motion constraint equation yields a line $\vec{v}=\left(v_{x}, v_{y}\right)^{T}$ space.

Figure 4.3: (a) and (b) Two fingerprint images, (c) optical flow determined by Horn-

Schunck method .64

Figure 5.1: Different kinds of fingerprint images with there coherence maps and detected low quality regions. $.68-69$

Figure 5.2: Result of single impression image enhancement for fingerprint with wet

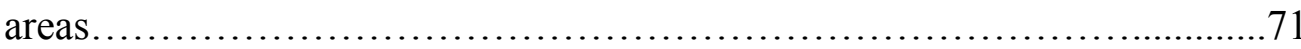

Figure 5.3: Result of single impression image enhancement for fingerprint with dry

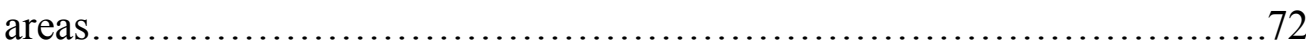

Figure 5.4: Result of single impression image enhancement for fingerprint with scars

Figure 5.5 Fingerprint image alignment with wet areas............................ 75

Figure 5.6 Fingerprint image alignment with dry areas..............................76

Figure 5.7: Thin Plate Splines (TPS) based fingerprint image alignment.............77-78

Figure 5.8 Binary level enhancement of fingerprint image with wet areas.............79-80

Figure 5.9 Binary level enhancement of fingerprint image with dry areas.............80-81

Figure 5.10 Grey level enhancement of fingerprint image with wet areas.............82-83 
Figure 5.11 Grey level enhancement of fingerprint image with dry areas............83-84

Figure 5.12 ROC curve plotting FAR and FRR .................................. 86 


\section{Chapter 1: Introduction}

\subsection{Biometric system}

Due to increase in frauds and crimes, personal authentication finds great importance in society today. Till the most recent time passwords, ID cards etc. were the most reliable modes of authentication. A legitimate user tries to keep simpler password which is based on 'what we know'. This weakens security as it can be easily guessed by an attacker. Another mean of authentication is ID cards which is based on 'what we have', can easily be lost or stolen. With fast growing technology and the world getting more and more digitized these means of authentication cannot be considered reliable and robust. Instead of 'what you know' and/or 'what you have', a robust authentication system needs the information about 'who you are'. Reliable and robust authentication system is required for secure access to vulnerable places such as research labs, government organizations, to transfer confidential data, applications like ATMs, workstations. Authentication systems are also required in forensic applications like prison security and crime investigations.

Humans have used body characteristics such as face, voice and gait for thousand of years to recognize each other [1]. Alphonse Bertillon more than a century ago introduced the idea of using body measurements for crime investigations. His idea faded due to discovery of distinctiveness of human fingerprints and since then fingerprints have been extensively used for authentication purposes. Every person possesses specific physiological and behavioral characteristics. Recognizing a person by determining the authenticity based on these characteristics is what is known as a biometric system. The 
system uses different biometric identifiers (e.g., fingerprint, iris, face, gait, hand geometry, signature) for automatically recognizing a person.

Table 1.1 summarizes existing biometrics identifiers and provides their description [2]

\begin{tabular}{|l|l|}
\hline Biometric Identifier & Description \\
\hline Fingerprint & Finger lines, pore structure \\
\hline Iris & Iris pattern \\
\hline Facial geometry & Distance of specific facial features (eyes, nose, mouth) \\
\hline Signature & Writing with pressure and speed differential \\
\hline Retina & On the back of an eye pattern of vein structure \\
\hline Hand geometry & Measurement of fingers and palm \\
\hline Finger geometry & Finger measurement \\
\hline Ear form & Dimensions of visible ear \\
\hline Voice & Tone or timbre \\
\hline DNA & DNA code as the carrier of human hereditary \\
\hline Odor & Chemical composition of one's odor \\
\hline Keyboard dynamics & Vein structure of the back of the hand \\
\hline
\end{tabular}

Table 1.1: Different biometric identifiers and their description

Characteristics of these biometric traits can be used as biometric characteristics as long as they fulfill the following requirements:

- Universality: Every person should have the characteristic.

- Distinctiveness: No two individuals should have same characteristics. 
- Permanence: Characteristics should not significantly change with period of time.

- Collectability: Characteristic should be easily collectable.

- Performance: Recognition accuracy and speed should give reasonable performance.

- Acceptability: People should be able to accept the use of characteristic.

- Circumvention: Characteristics should not be easily spoofable.

Figure 1.1 shows a generic biometrics system which can be divided into 5 subsystems:

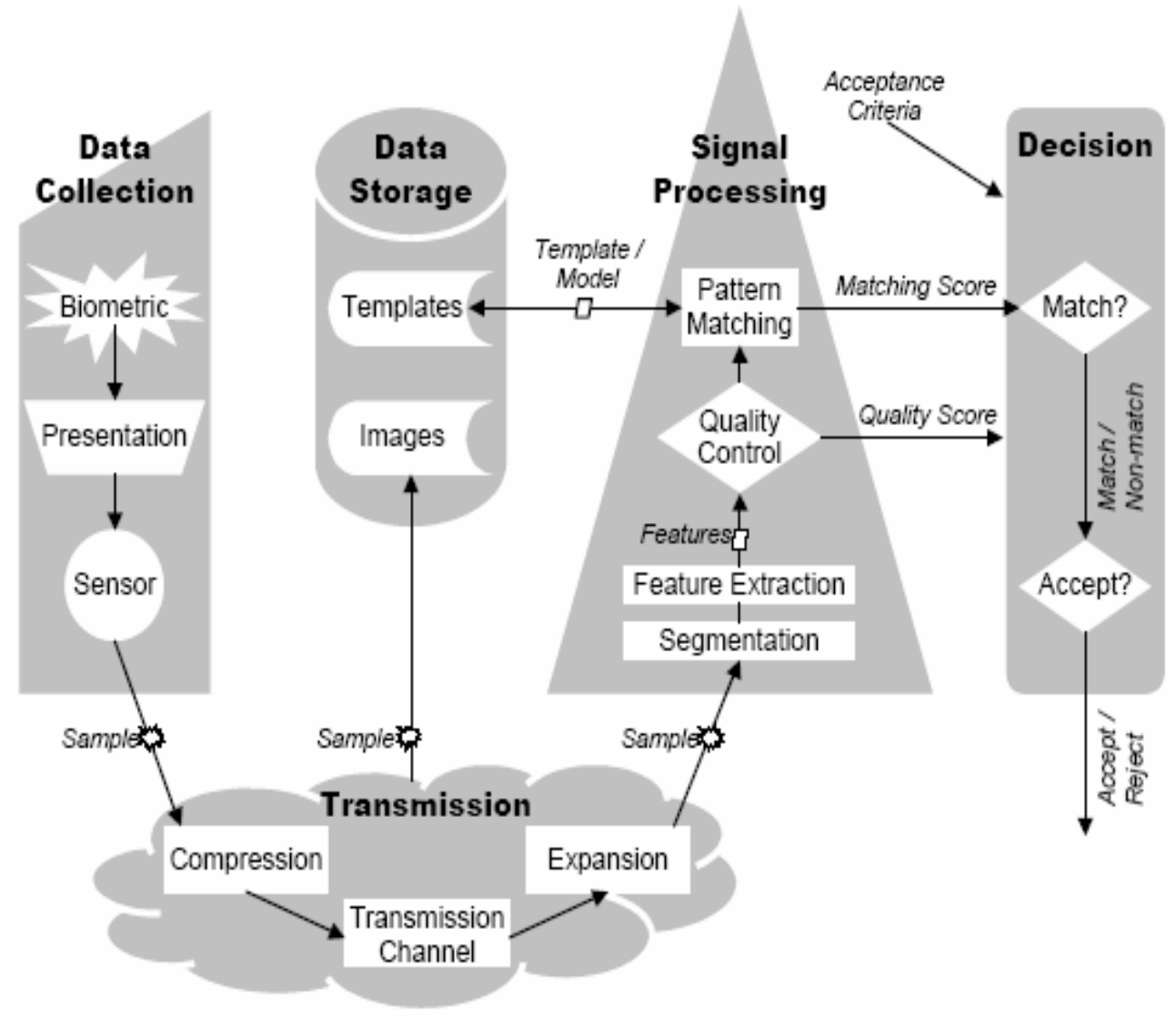

Figure 1.1 High level schematic of a generic biometric system [59]

Data Collection: This subsystem uses sensor or camera to acquire the image of the biometric trait of the user. 
Transmission: This subsystem transmits the data collected from data collection module after compressing it, to the signal processing and data storage module.

Data Storage: This module is used for storing the image and template of the user.

Signal Processing: This is the most important module of the system. It performs feature extraction by image processing techniques and pattern matching operations.

Decision: This module performs identification or verification by using the match scores.

Biometric system functionality: In a typical biometric system first of all acquisition takes place which means acquiring of biometric data from the user. Then comes the feature extraction part which involves use of various image processing applications. Finally matching is done with template feature sets in the database. All biometrics systems can operate in two modes:

i. Verification (authentication): verification system tries to find out whether the user is genuine or an imposter. It tries to authenticate the users identity by comparing the captured biometric characteristic with the one pre-stored in the database with his or her name. Therefore it performs one-to-one comparison, determining whether identity claimed by the user is true or not. Figure 1.2 shows the block diagram of verification mode. 


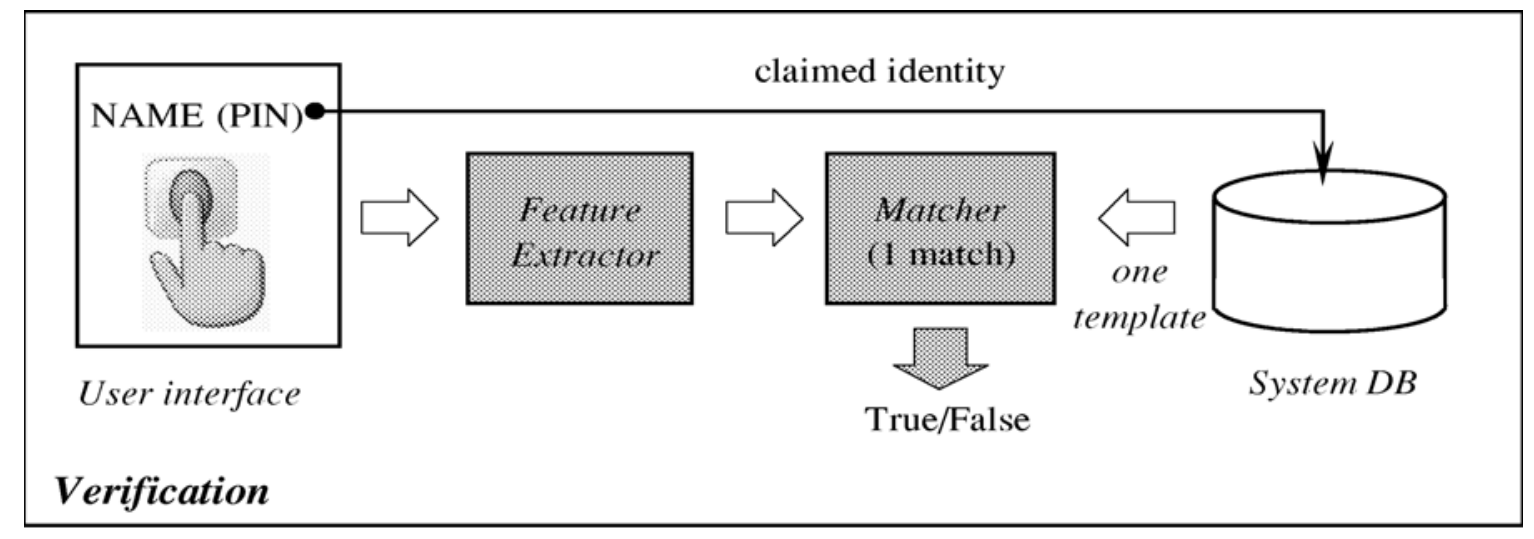

Figure 1.2 Verification mode [1].

ii. Identification (recognition): identification mode tries to recognize a person in the whole database. System tries to identify a person by comparing the captured biometric characteristic with the whole database and finding the best match. User does not claim his or her identity in this mode. Therefore, it performs one-tomany comparisons determining the person's identity. Figure 1.3 shows the block diagram of identification mode:

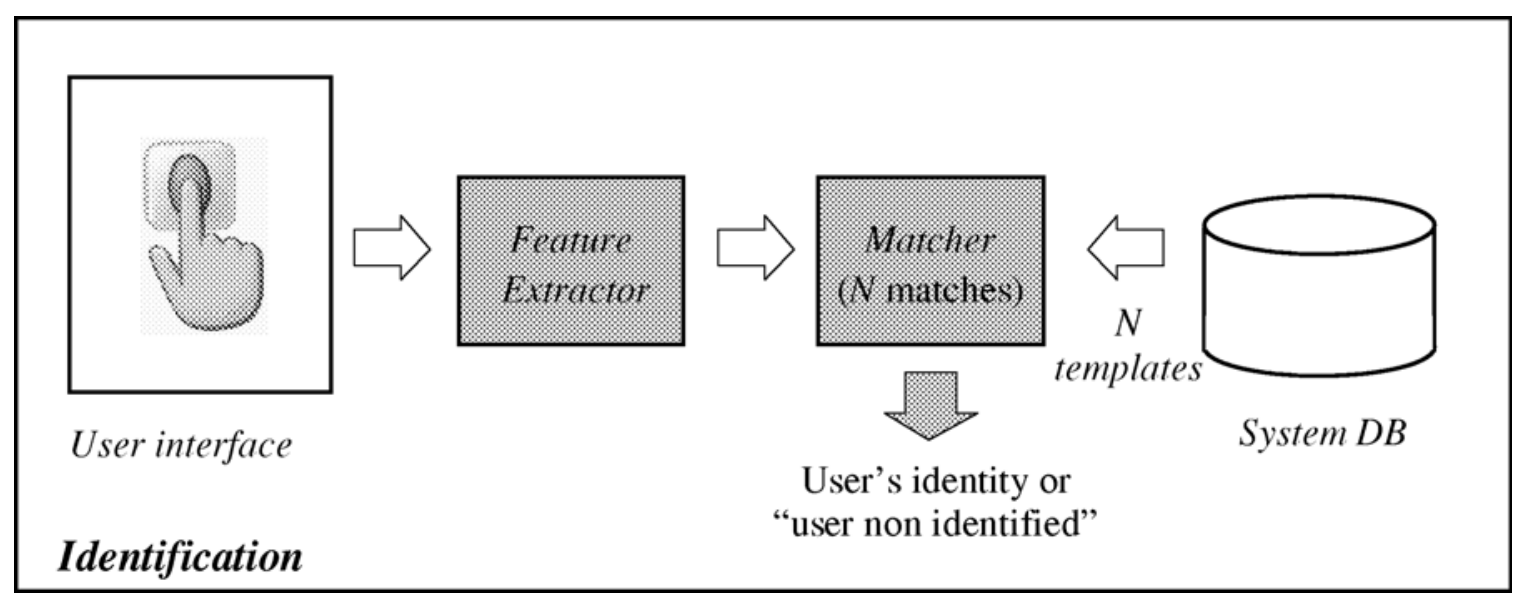

Figure 1.3 Identification mode [1].

As performance measures of accuracy and reliability False Accept Rate (FAR) and False Reject Rate (FRR) are used. The FAR is the rate at which the unauthorized users are accepted by the system, whereas, the FRR is the rate at which the authorized users are 
rejected by the system. For a biometric system to be more reliable and accurate, both the FAR and the FRR should be as low as possible.

\subsection{Fingerprint}

Fingerprint is one of the oldest and most widely used biometric traits. Awareness of the individuality of fingerprints goes back for hundreds of years but without any scientific basis [3]. It was not until late sixteenth century that the modern scientific fingerprint technology was first initiated [3]. Figure 1.4 shows the features of a fingerprint:

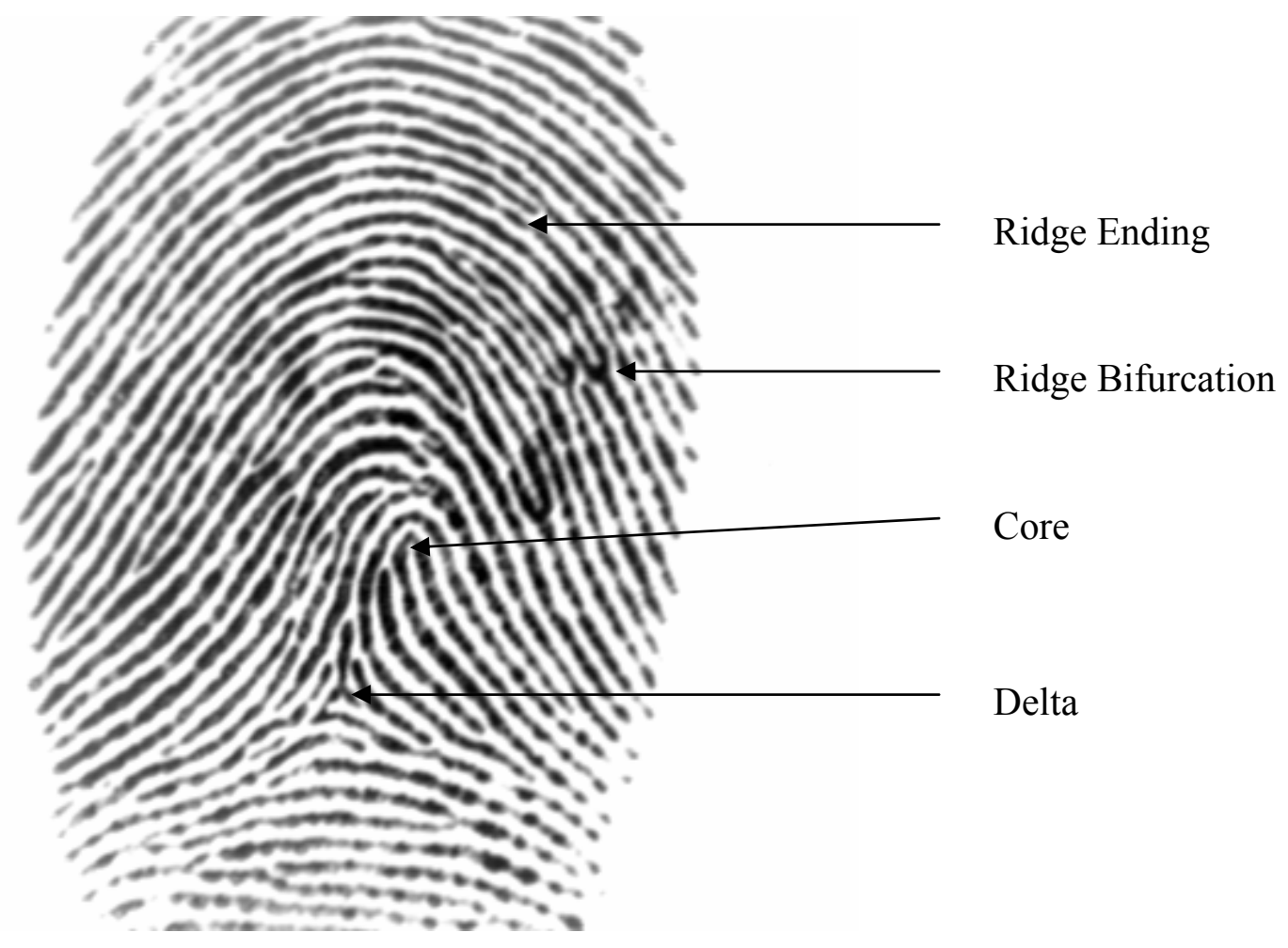

Figure 1.4: Features of a fingerprint

In earlier days, to acquire fingerprint image ink impression of the fingertip on a paper was used. Today a, wide range of sensors are available commercially to get digital 
fingerprint image. Due to low cost and simplicity of operations, fingerprints are widely used these days for user authentication purpose.

Fingerprints are well known for their uniqueness. Even twins do not have identical fingerprints. Fingerprints are characterized by ridges and valleys, where ridges are the dark and valleys are bright as shown in Figure 1.4. It is due to the ridge pattern and local ridge characteristics that uniqueness of fingerprints is determined. Global characteristics of fingerprint are defined by the flow of ridge pattern in the central part of the image. Ridge flow has different patterns and these patterns are classified into three singular regions: loop, delta and whorl. Most important global features are core and delta points. Core is defined as north most point of the innermost ridge of the fingerprint. Delta points are the points where three patterns deviate [4].

The local ridge characteristics are defined by minutiae which represent the discontinuities in the ridge structure. Ridge endings and ridge bifurcations as shown in Figure 1.4 are the two discontinuities which are considered as minutiae points. While performing fingerprint matching, location and distribution of minutiae points are considered.

Fingerprint matching is categorized as minutiae based matching and correlation based matching. Minutiae based matching uses relative position of minutiae points after extracting them whereas correlation based matching uses global ridge pattern for matching two fingerprints. Fingerprint recognition system is very authentic and reliable; therefore it is very widely used commercially these days. 


\subsubsection{Fingerprint Quality Assessment}

Fingerprints are widely used in many personal identification systems due to its permanence and uniqueness. Fingerprints are being increasingly used not only in forensic environments, but also in large number of civilian applications such as access control or on-line identification [5].

Performance of a fingerprint recognition system depends heavily on the quality of fingerprint image. Several factors determine the quality of fingerprint image: skin conditions (e.g. wetness, dryness, dirtiness, temporary or permanent cuts or bruises), sensor conditions (e.g. dirtiness, noise, size), user cooperation etc [5]. Poor image quality leads to many pseudo-minutiae. This degrades the performance of fingerprint recognition system. Thus, it is very important to estimate the quality and validity of captured fingerprint image in order to assure good performance of fingerprint recognition system. Examples of good and poor quality fingerprint images are shown in Figure 1.5. 


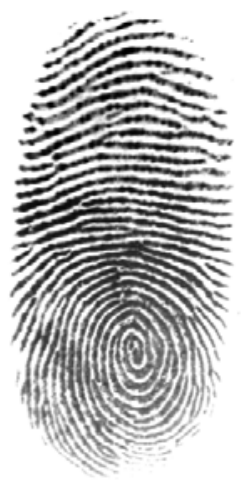

(a)

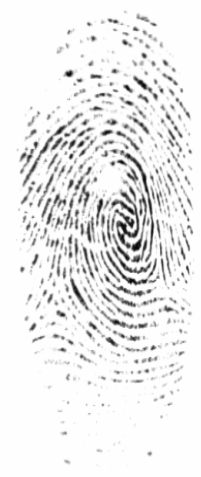

(b)

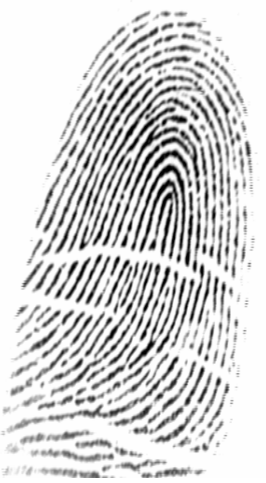

(c)

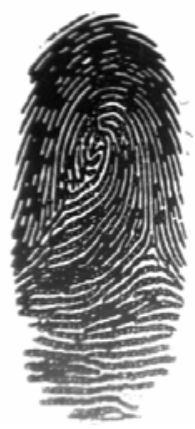

(d)

Figure 1.5: (a) Good quality image, (b) Fingerprint with dry areas, (c) Fingerprint with scars, (d) Fingerprint with wet areas.

Fernandez et al. [5] reviewed the schemes for fingerprint image quality computation. Fingerprint image quality schemes can be divided into two groups: (i) those that use the local features of the image, (ii) those that use global features of the image. Methods that use local features for quality assessment of fingerprint image divide image into nonoverlapping blocks and extract features from those blocks. Local measure of quality is generated and finally quality of the image is decided. There are three methods according to the review which use local features of the image:

1) Based on orientation field: Hong et al. [6] modeled ridges and valleys as a sinusoidal-shaped wave along the direction normal to the local ridge orientation and extracted the amplitude, frequency and variance of the wave. They classify each block as recoverable and unrecoverable based on that parameters extracted. If the number of unrecoverable blocks is greater than a particular threshold then image is classified as of poor quality. 
Lim et al. [7] compute orientation certainty level, ridge frequency, ridge thickness and ridge to valley thickness ration of each block, and by thresholding these four features, blocks are labeled as "good", "undetermined", "bad", "blank". Finally a quality score is computed based on the percentages of first three labels.

According to a scheme proposed by Chen et al. [8] it measures the spatial coherence using the intensity gradient. The orientation coherence of each block is computed and finally a local quality score is computed by averaging the coherence of each block.

2) Based on Gabor filters: According to scheme proposed by Shen et al. [9] each block is filtered using a Gabor filter with $\mathrm{m}$ different responses. Response of $\mathrm{m}$ filter responses is same if the block is of poor quality otherwise one or several filtering responses is larger than the others if block is of good quality. To determine the quality of block standard deviation of $\mathrm{m}$ filter responses is used. A quality index of whole image is computed as the percentages of good quality blocks and finally a threshold on quality index is used to determine the quality of the image.

3) Based on pixel intensity: Ratha et al. [10] proposed a method in which each block is classified as directional and non-directional by calculating sum of intensity differences $D_{d}(i, j)$ between a pixel $(i, j)$ and $n$ pixels selected along a line segment of orientation $\mathrm{d}$ and is computed for $\mathrm{m}$ different orientations. Histogram of $D_{d}(i, j)$ values is obtained for each orientation and each pixel in a block. If only one of the $\mathrm{m}$ histograms has maximum value greater than a particular threshold then the block is "directional" otherwise "non directional". 
An overall quality index is computed by assigning relative weight to each block based on its distance to the foreground centroid. A predefined threshold for quality index determines the quality of the image.

There are two methods that use global features to determine the quality of a fingerprint image;

1) Based on orientation field: Lim et al. [7] presented two features to analyze the global structure of the image. For first feature, abrupt orientation changes between blocks are accumulated and mapped into a global orientation score and it checks the continuity of orientation field. For computing second feature which checks the uniformity of frequency field, standard deviation of the ridge-to-valley thickness ratio is computed and mapped into a global score. Large deviation indicated low image quality as separation in ridges and valleys in good quality images is more uniform than in poor quality images.

2) Based on Power Spectrum: Chen et al. [8] analyzed the global structure by computing 2D Discrete Fourier Transform (DFT). An annular region with radius ranging between the minimum and maximum typical ridge frequency values is defined as region of interest (ROI). In good quality image energy will be more concentrated in ring patterns within the ROI. A set of bandpass filters is constructed and amount of energy in ring-shaped bands is computed to define a global quality index. Good quality images will have the energy concentrated in few bands. 
Lim et al. [28] proposed a fingerprint quality and validity analysis technique. According to them a fingerprint image quality analysis should examine both the local (main texturelike patterns of ridges and valleys) and the global structures (puts the ridges and valleys into a smooth flow). For local analysis, fingerprint image is partitioned into blocks of size $32 \times 32$. In a small block consistent ridge orientation and the appropriate ridge and valley structure are two distinguishable local characteristics.

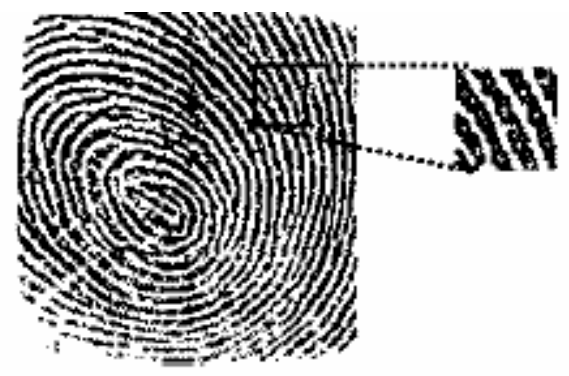

Figure 1.6: A typical texture-like ridge block [28].

By performing Principal Component Analysis (PCA) on the image gradients in an image block, an orthogonal basis for an image block can be performed by finding its eigenvalues and eigenvectors. Energy concentration along the dominant direction with two vectors pointing to the normal and tangential direction of the average ridge flow is given by ratio between the two eigenvalues. The covariance matrix $\mathrm{C}$ of the gradient vector for a $\mathrm{N}$ point image block is given by

$C=E\left\{\left[\begin{array}{l}d x \\ d y\end{array}\right]\left[\begin{array}{ll}d x d y\end{array}\right]\right\}=\left[\begin{array}{l}a c \\ c b\end{array}\right]$

where $E\{\bullet\}=\frac{1}{N} \sum_{N} \bullet$

For a fingerprint image block, the ratio between eigenvalues $\lambda_{\min }$ and $\lambda_{\max }$ is given by: 
$o c l=\frac{\lambda_{\min }}{\lambda_{\max }}=\frac{\left[(a+b)-\sqrt{(a-b)^{2}+4 c^{2}}\right] / / 2}{\left[(a+b)+\sqrt{(a-b)^{2}+4 c^{2}}\right] / /}$

It gives an indication how strong the energy is concentrated along the ridge-valley orientation. Total quality score for local analysis is given by:

$S_{L}=\frac{T_{G}+0.5 \times T_{U}}{T_{G}+T_{U}+T_{B}}$

$T_{G}, T_{U}, T_{B}$ are the total number of good, undetermined and bad quality blocks.

For global analysis, orientation change along each horizontal row and each vertical column of image block is examined, and it is observed that there are smooth changes in orientation in a valid fingerprint and abrupt changes in the region for noisy fingerprint. The abrupt change in orientation is accumulated and is mapped into global orientation score $\left(\mathrm{S}_{\mathrm{GO}}\right)$ with highest quality score of 1 and lowest 0 . This provides an efficient way of investigating whether a fingerprint image possesses a global orientation structure or not. Ratio of ridge thickness and valley thickness is computed for each image block. The standard deviation gives and indication of the quality the image carries. Large deviation from the mean ratio value can be an indicator of bad or good quality and is mapped into global score $\mathrm{S}_{\mathrm{GR}}$ ranging from 0 to 1 .

Finally, total image quality score is given by:

$Q S=\left(\alpha_{1} S_{L}+\alpha_{2} S_{G O}+\alpha_{3} S_{G R}\right) \times \frac{\min \left(T-T_{B L}, A_{\min }\right)}{A_{\min }}$

and $\alpha_{1}+\alpha_{2}+\alpha_{3}=1$. 
$\alpha_{1}, \alpha_{2}, \alpha_{3}$ are the coefficients of quality analysis score. $T_{B L}$ is the number of blank blocks obtained from adaptive thresholding. $T$ is the sum of $T_{G}, T_{U}, T_{B}$ and $T_{B L} . A_{\min }$ is the required minimum number of blocks that identified as foreground images to avoid insufficient of information.

Aguilar et al. [29] assess the quality of a fingerprint image based on the energy distribution in the power spectrum. In a good quality fingerprint image there is a strong ring pattern in the power spectrum, indicating a dominant frequency band associated with the period of the ridges. Conversely, there is a diffused power spectrum in poor quality image due to unclear and non-uniformly spaced ridges.

A region of interest (ROI) in the power spectrum is defined to be ring shaped band with radius ranging from the minimum to the maximum observed frequency of ridges. Good quality fingerprint image presents a strong ring pattern in the power spectrum (Fig. 1.7 (c)) whereas, poor quality image (Fig. 1.7 (a)) has more diffused ring pattern in the power spectrum as shown in Figure 1.7. 

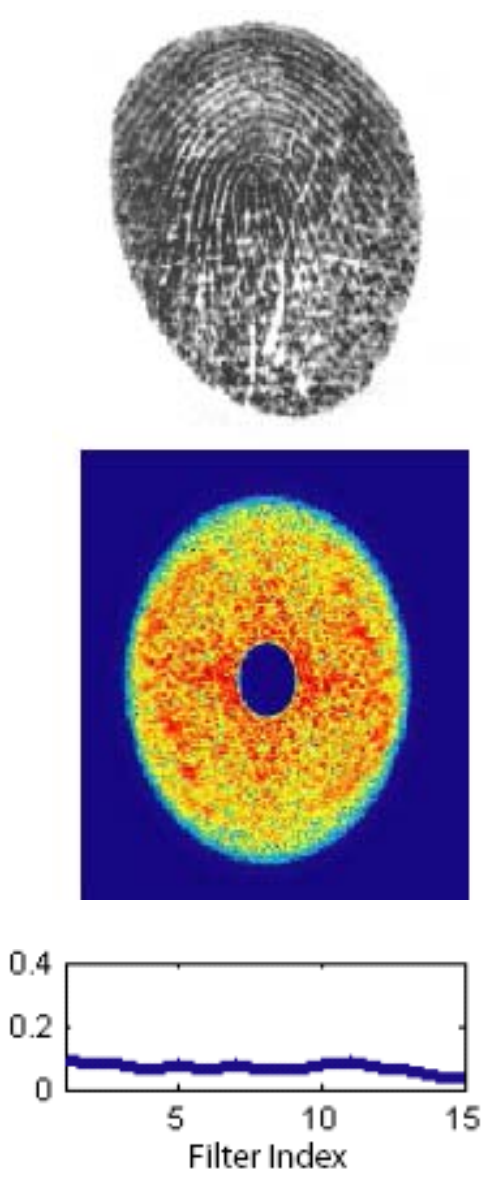

(a)
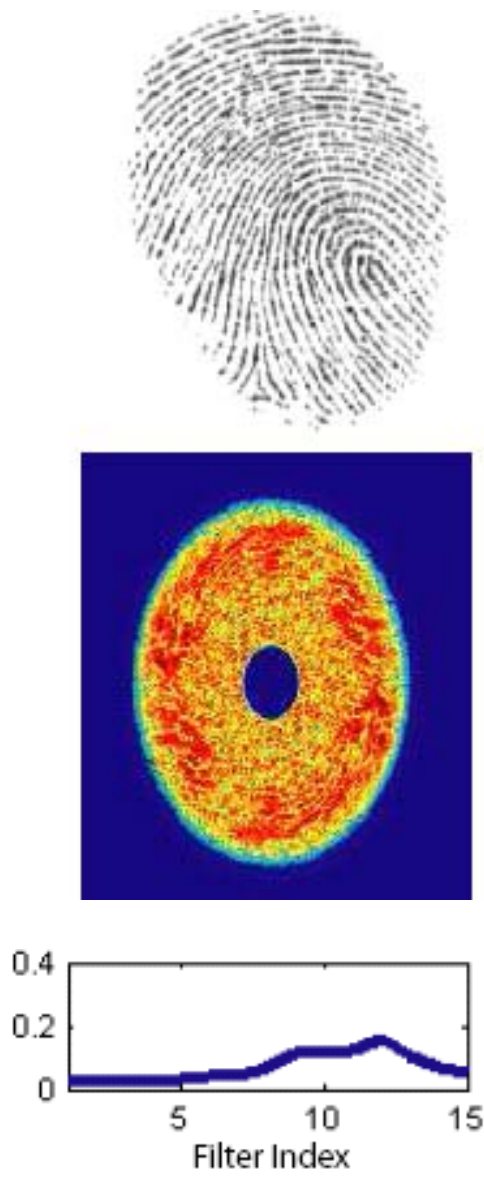

(b)
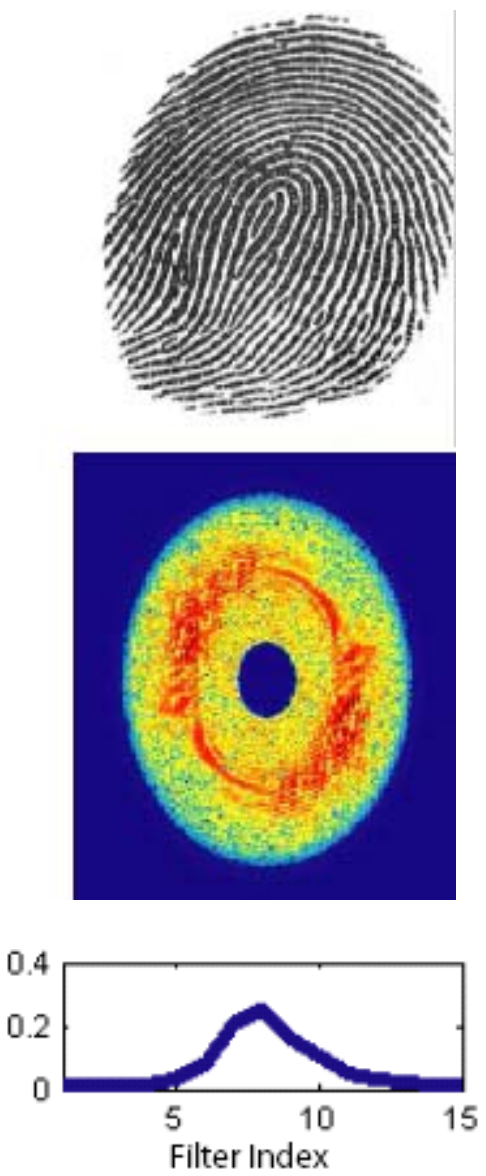

(c)

Figure 1.7: Fingerprint images with their power spectrum [29].

To extract the energy in a number of ring-shaped concentric sectors in the power spectrum multiple bandpass filters are designed. The global quality index is defined in terms of the energy concentration across these sectors within the ROI. Third row of Fig. 2.2 shows the distribution of normalized energy across the bandpass filter. The quality measure is based on the entropy of this distribution, which is normalized to the range [0, 1] and is more peaked as the image quality improves from (a) to (c). 


\subsubsection{Fingerprint Deformation}

Most of the fingerprint matching systems are based on minutiae, which are ridge endings or ridge bifurcations. There are lot of complications in minutiae based matching system. Both sets may have false, missed, and displaced minutiae caused by the imperfection in minutiae extraction stage. Two fingerprints may be from different parts of the finger, therefore there may have partial overlap. Two fingerprints may be translated, rotated or scaled. Finally, there may be non-linear elastic deformation present in the fingerprints, this is very challenging problem.

This deformation are due to several factors such as: the amount of pressure applied by the user on sensor, the disposition of the user, orientation of the sensor with respect to finger, the motion of the finger prior to its placement on the sensor, the elasticity of the skin etc. [11]. Basically, non-linear deformation occurs at the time of image acquisition.

Most of the matching algorithms align fingerprints using affine transform, but due to presence of non-linear deformation, alignment is not quite good. Figure 1.8 shows alignment of two fingerprint images after affine transform. 


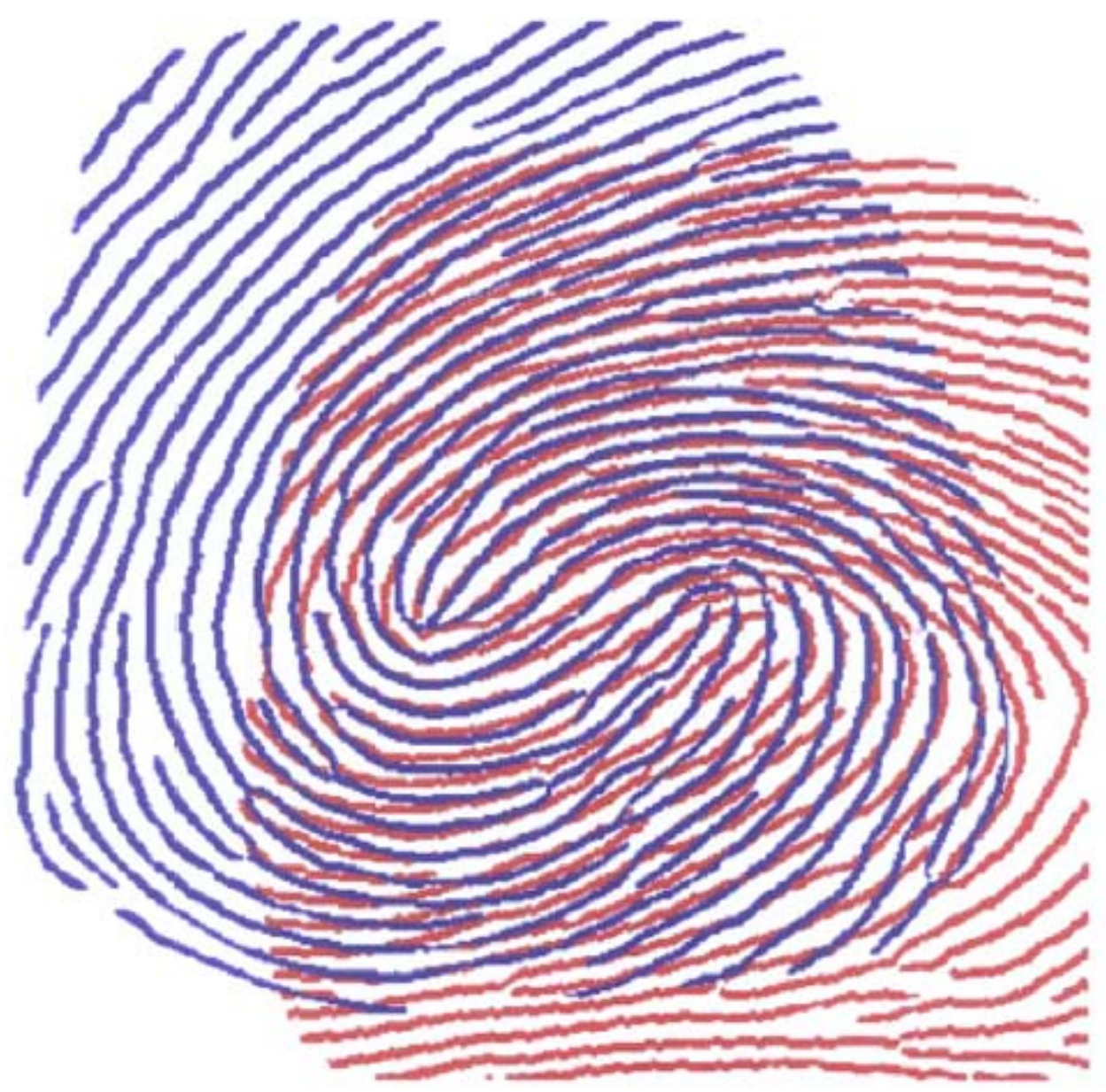

Figure 1.8: Alignment of two impressions of same finger after affine transform [11].

Bazen et al. [31] proposed a novel minutiae matching method that describes elastic distortions in fingerprints by means of a thin-plate spline model, which is estimated using a local and a global matching stage. The matching algorithm proposed by them estimates the non-linear transformations in two stages. First, the local matching, determines which minutiae possibly match, based on local similarity measures. Next, the global matching, that uses the possible correspondences to estimate the global non-rigid transformations. 
Local Matching: Minutiae in template and test fingerprints are presented by parameters, pixel coordinates $(x, y)$ and orientation of the minutiae $\theta$ i.e. $(x, y, \theta)$. There is a minutia neighborhood for each minutia which consists of minutiae itself and its two neighboring minutiae.

The local matching algorithm compares each minutia neighborhood in the template fingerprint to the minutia neighborhood in the test fingerprint. For this, first of all two structures are aligned by optimal rotation, translation and scaling which are determined using a least square algorithm. Next, a local matching decision is made by comparing the scaling, the sum of squared distances between the corresponding minutiae, and the differences of orientation to a threshold. Matched pair of minutiae neighborhoods and the translation, rotation, and scaling parameters are stored. Finally, a list of corresponding minutiae neighborhood pairs is obtained, which does not contain all true correspondences. However, its size gives a first indication of the degree of similarity of the two fingerprints.

Global Matching: In the global matching stage, for each matching pair the number of pairs of which the registration parameters differ less than a certain threshold are determined to select the largest group of pairs that share approximately the same registration parameters. The transformation that optimally registers the selected minutiae in the test set to the corresponding in the template set is calculated in a least square sense. 
For elastically deformed fingerprints accurate registration $(t, r, s)$ does not exist, so they will not register well. Hence, elastic registration has to be used to compensate for plastic distortions. Authors, proposed this-plate spline (TPS) model for elastic deformations. Given the displacements of landmark points, the TPS model interpolates those points, while maintaining the maximal smoothness, which is represented by the bending energy of thin metal plate. The displacement at each landmark point $(\mathrm{x}, \mathrm{y})$ is represented by an additional parameter z-coordinate, and, for each point, the thin metal plate is fixed at position $(\mathrm{x}, \mathrm{y}, \mathrm{z})$. The TPS model for one of the transformed coordinates is given by parameters a and $\mathbf{w}$ :

$f(x, y)=a_{1}+a_{2} x+a_{3} y+\sum_{i=1}^{n} w_{i} U\left(\left|P_{i}-(x, y)\right|\right)$,

where, $U(r)=r^{2} \log r$ is the basis function, $\mathbf{a}$ is the affine part of the transformation, $\mathbf{w}$ gives an additional non-linear deformation, $P_{i}$ is the landmark to be interpolated, and $\mathrm{n}$ is the number of landmarks. The TPS parameter that minimizes the bending energy can be found by solving a set of linear equations:

$K w+P a=v$,

$P^{T} w=0$

The smoothness is controlled by a parameter $\lambda$, which weights the optimization of landmark distance and smoothness. For, equal isotropic errors at all landmarks, the optimal TPS parameters can be found by solving the following system of equations:

$$
\begin{aligned}
& (K+\lambda I) w+P a=v, \\
& P^{T} w=0
\end{aligned}
$$

where, I is the $\mathrm{n} \mathrm{n}$ identity matrix. 
The TPS model is fitted in a number of iterations. Initial model is fitted to the minutiae in the minutia neighborhood pairs that were found in the local matching stage. Then, it is fitted to the largest group of pairs that share approximately same registration parameters. This is repeated with a decreasing threshold $r_{0}$ which is Euclidean distance between two minutiae, until the model has converged to the final state. Finally, the matching score $\mathrm{S}$ is calculated by:

$$
S=\frac{n_{\text {match }}^{2}}{n_{1} n_{2}}
$$

Ross et al. [11] developed an average deformation of using thin plate splines (TPS) model, of each template impression by comparing it with rest of the impressions of that finger. The authors compute the average deformation model using fingerprints impressions originating from the same finger, where in the technique described earlier, authors, use a TPS model to align every pair impression, even if they are from two different fingers.

Let $F$ be the warping function which takes $I_{0}$ to $I_{1}$, which denote two fingerprints corresponding to the same finger. Let, there be a pair of point patterns with known correspondences, say, $U=\left(u_{1}, u_{2}, \ldots \ldots \ldots . u_{m}\right)^{T}$ and $V=\left(v_{1}, v_{2}, \ldots \ldots \ldots v_{m}\right)^{T}$ that need to be registered where $m$ is the total number of corresponding points. The warping function $F$ is given by the conditions

$$
F\left(u_{j}\right)=v_{j} \quad \text { for } \mathrm{j}=1,2,3, \ldots \ldots \ldots \ldots \ldots \ldots \ldots, \mathrm{m} .
$$

The average deformation at each point is defined as: 
$\bar{F}(u)=\frac{1}{N} \sum_{i=1}^{N} F_{i}(u)$

if we have $\mathrm{N}$ pairs of correspondences given by $\left(U_{i}, V_{i}\right)_{i=1}^{N}$, and the covariance matrix corresponding to the deformation is given by

$D_{F}(u)=\frac{1}{N} \sum_{i=1}^{N}\left(F_{i}(u)-\bar{F}(u)\right) \cdot\left(F_{i}(u)-\bar{F}(u)\right)^{T}$.

The deformation model is given in terms of warping function $F_{i}(u)$ as

$F_{i}(u)=c_{i}+A_{i} \cdot u+W_{i}^{T} s(u)$,

where u $\in \mathrm{S}, c_{i}$ is a $2 \times 1$ vector, $A_{i}$ is a $2 \times 2$ matrix, $W_{i}^{T}$ is a $m_{i} \times 2$ matrix, $s(u)=\left(\sigma\left(u-u_{i, 1}\right), \sigma\left(u-u_{i, 2}\right), \ldots \ldots \ldots . . .\left(u-u_{i, m_{i}}\right)\right)$ and

$\sigma(u)= \begin{cases}\|u\|^{2} \log (\|u\|) & \|u\|>0, \\ 0 & \|u\|=0,\end{cases}$

From the above equations, TPS model can be obtained by the matrix equation.

$$
\left[\begin{array}{lll}
H & 1_{m_{i}} & U_{i} \\
1_{m_{i}}^{T} & 0 & 0 \\
U_{i}^{T} & 0 & 0
\end{array}\right]\left[\begin{array}{l}
W_{i} \\
c_{i}^{T} \\
A_{i}^{T}
\end{array}\right]=\left[\begin{array}{l}
V_{i} \\
0 \\
0
\end{array}\right]
$$

The covariance matrix, defined at each pixel, is a measure of variability associated with the estimated warping functions around the average.

Next, let there are $\mathrm{N}$ impressions of a finger $T_{1}, T_{2}, \ldots \ldots \ldots \ldots \ldots . . . T_{N}$. To find out which one of these fingerprints should be used as reference image for estimating the average deformation of this finger, all possible pairings are considered. The average deformations 
$\bar{F}_{T_{i}}$ and the covariance matrix $D_{\bar{F}_{T_{i}}}$ are obtained. Author defines the $i$ th index of deformation as $\Phi_{i}=\frac{1}{|S|} \sum_{u=1}^{|S|} \Phi\left(D_{\bar{F}_{T_{i}}(u)}\right)$

Low (high) values of the index of deformation indicate that the warping functions are similar (dissimilar) to each other. Figure 1.9 shows the average deformation model of a finger as distortion on a reference grid.

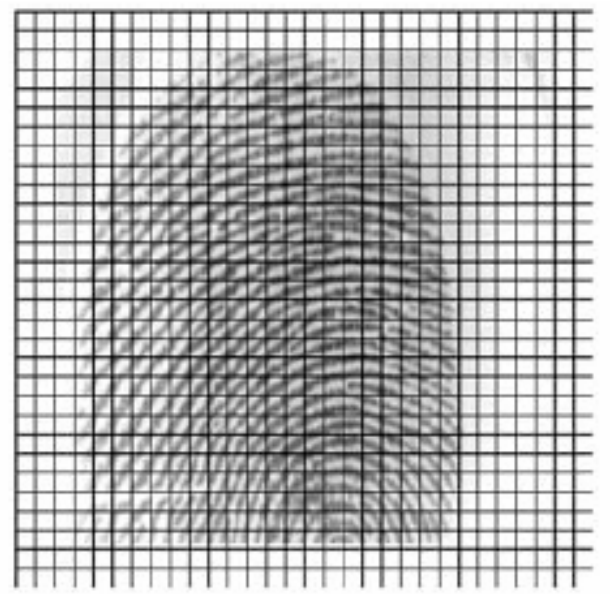

(a)

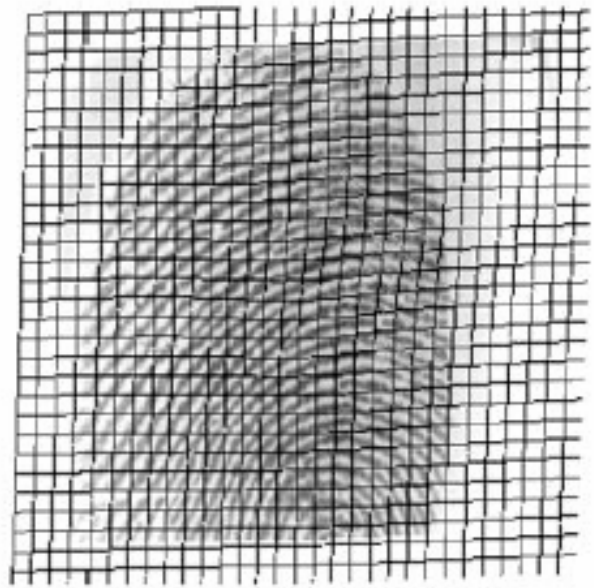

(b)

Figure 1.9: (a) Original impression, (b) Average deformation model on a reference grid [11]

where, $n_{\text {match }}$ is the number of matching minutiae, $n_{1}$ is the number of minutiae in the test fingerprint and $n_{2}$ is the number of minutiae in the template fingerprint. 


\subsubsection{Fingerprint Enhancement}

Fingerprints are widely used for authentication purpose due to their proved distinctiveness. This uniqueness comes from unique local characteristics ridge bifurcations and ridge endings known as minutiae as shown in figure 1.10. Most of the fingerprint matching algorithms use details of these minutiae points to compare two fingerprint images. The performance of these algorithms depends significantly on the quality of fingerprint images. Quality of fingerprint images differ due to many factors like imaging conditions, type of sensor used, acquisition conditions, age, skin characteristics etc.

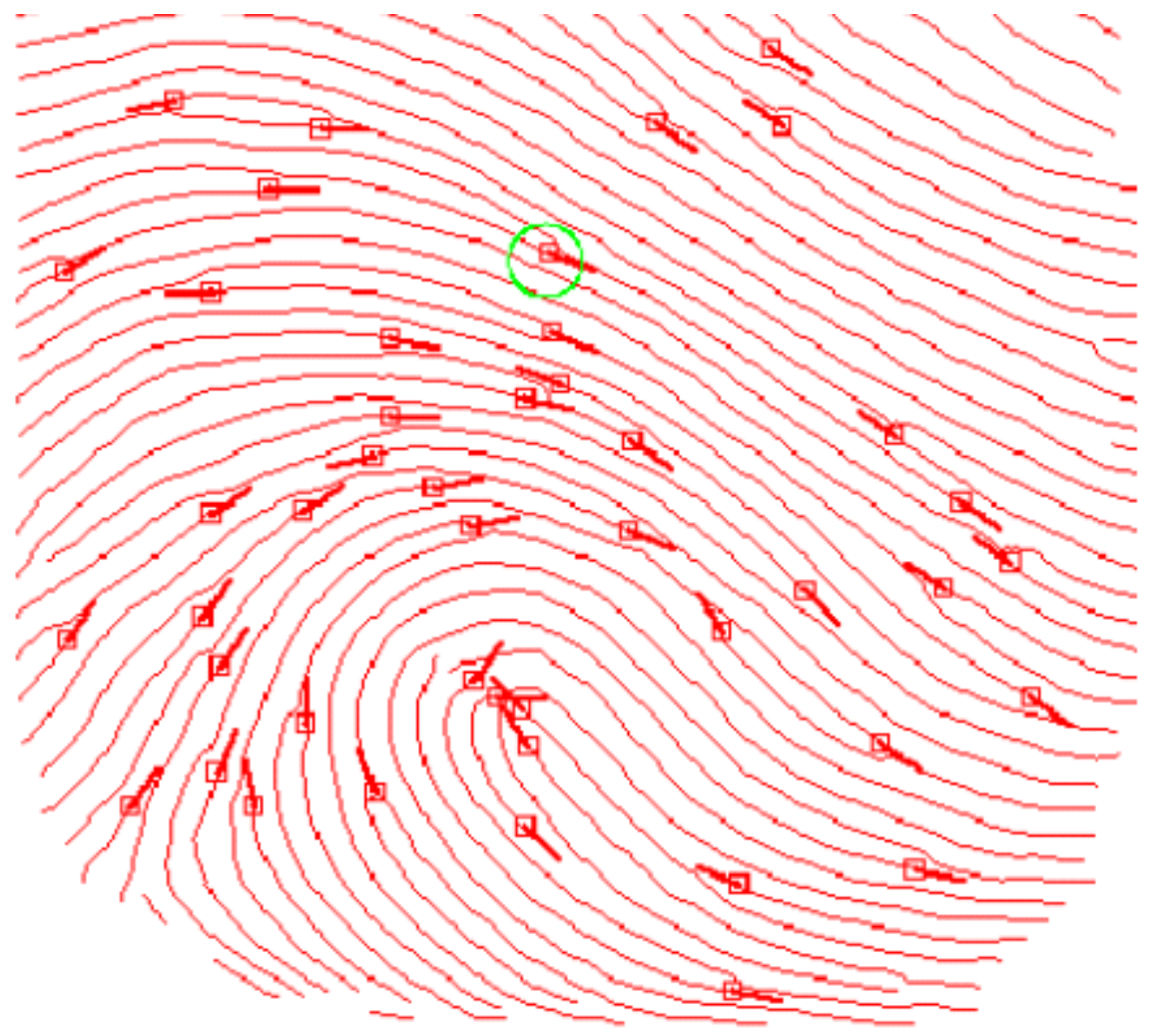

Figure 1.10: Fingerprint with minutiae points [12]. 
Minutiae from poor quality images contain many pseudo-minutiae. These pseudominutiae can affect the performance of a matching algorithm significantly. Therefore, we need an effective fingerprint image enhancement procedure to eliminate these pseudominutiae and thus improve the performance of fingerprint matching algorithm.

The main aim of enhancement algorithm is to reduce unwanted noise in the image as much as possible and to enhance the ridges in the affected areas. There are numbers of fingerprint enhancement procedures proposed in the literature. Most of the fingerprint enhancement techniques make the use of filters for enhancement. The most widely used technique is based on the use of contextual filters [3]. Instead of using single filter for the entire image, different filters are used for different areas of the image. Filters are designed according to the local ridge orientation and local ridge frequency as they differ in different areas of a fingerprint. An appropriate filter that is tuned to the local ridge frequency and orientation can effectively remove the undesired noise and preserve the true ridge and valley structure [3].

Hong et al. [13] proposed a fingerprint enhancement algorithm which decomposes the input fingerprint image into a set of filtered images. The orientation field and a quality mask which distinguishes recoverable and unrecoverable region are estimated from the set of filtered images. Algorithm proposed by them basically consists of two stages: (i) orientation field estimation, and (ii) enhancement. 
Gabor filters as bandpass filters are used to remove the noise and preserve true ridge/valley structures as gabor filters have both frequency-selective and orientationselective properties and have optimal joint resolution in both spatial and frequency domains. For each input image 8 gabor filters with orientations $0^{0}, 22.5^{0}, 45^{0}, 67.5^{0}$, $90^{0}, 112.5^{\circ}, 135^{\circ}, 157.5^{\circ}$ and radial bandwidth of 2.5 octaves are applied to obtain 8 filtered images. Local orientation field is estimated for extracting ridges as; in the filtered images grey level values on ridges attain their local maxima along the direction that is orthogonal to local ridges. Now, two filters are applied to the filtered image to adaptively accentuate the local maximum grey level values along the normal direction of the local ridge orientation. The ridges in the input fingerprint can be efficiently located by tuning the mask width to the width of the local ridges.

For enhancement, area of each connected component appearing in the ridge map is computed and if area is less that a threshold then it is labeled as background otherwise, connected component is break into a set of short line segments. If each of short line segment is between a pair of narrow parallel ridges, it is labeled as true ridge otherwise background. After this most of the spurious ridges are removed.

Now, ridge map of each filtered image is divided into blocks of size $8 \times 8$. If each block have enough ridge pixels appearing around the block, it is labeled as foreground otherwise background. A binary block map is obtained in which 1 represents the presence of ridge and 0 as non-ridge. Now, for each block, all eight filtered images are examined and coarse-level ridge map is obtained by following for rules: 
1. If only one of the eight binary block map at pixel (x,y) has the value 1 and this pixel belongs to a connected component of size $\mathrm{K}, \mathrm{K}>\mathrm{T}$ block, then the pixel values of the corresponding block in the coarse-level ridge map are duplicated from the associated ridge map. This block is recoverable.

2. If more than one binary block map a pixel $(x, y)$ has the value 1 and the associated local ridge orientations are not orthogonal to one another, the pixel values of the corresponding block in the coarse-level ridge map are taken as average values of the associated ridge maps. This block is also recoverable.

3. If more than one binary block map a pixel (x,y) has the value 1 and the associated local ridge orientations may be orthogonal to one another, and only one pixel with the value 1 resides in a connected component of size larger than certain threshold $\mathrm{T}_{\text {block}}$, then the pixel values of the corresponding block in the coarse-level ridge map are duplicated from the ridge map associated with the largest connected component. This block is also recoverable.

4. If the above conditions are not satisfied, then the block is assigned a label 1 to indicate that it is unrecoverable.

The orientation field of a given input fingerprint image can now be reliably estimated from the coarse-level ridge map by ignoring the unrecoverable region. Using the estimated orientation field, the input fingerprint image can then be adaptively enhanced $[13,30]$. 
In a method proposed by Almansa and Lindberg [14] two mechanisms, shape-adapted smoothing based on second moment descriptors and automatic scale selection based on normalized derivatives are used for fingerprint enhancement. First mechanism adapts the smoothing operation to the local ridge structures, which allows interrupted ridges to be joined without destroying singularities such as branching points. Second mechanism estimate local ridge width and adapts the amount of smoothing to the local amount of noise.

Meihua and Zhengming [15] proposed fingerprint enhancement based on Edge-directed Diffusion. This model adapts an image structure tensor like coherence enhancement diffusion for edge orientation, and defines eigenvalues based on edge orientation such that the new tensor has positive diffusivity in gradient direction. To construct the edgedirected diffusion model authors introduced the following theorem.

Assume $u_{0}$ is the image to be enhanced, $\mathrm{D}$ is the diffusion tensor of size $2 \times 2, p_{1}, p_{2}$ are its normalized orthogonal eigenvectors, where $p_{1}$ is the gradient direction and $p_{2}$ is the edge direction. $\lambda_{1}, \lambda_{2}$ are its corresponding eigenvalues, consider the following diffusion problem :

$\left\{\begin{array}{l}\partial_{t} u=\operatorname{div}[D \nabla u] \\ u(0, x, y)=u_{0}(x, y)\end{array}\right.$

Then model 2.8 approximately smoothes $u_{0}$ in the direction $p_{1}$ and $p_{2}$ with speed $\lambda_{1}, \lambda_{2}$ respectively. $\nabla u_{\sigma}$ is good operator to detecting edge, but is unsuited for finding parallel structures. $\nabla u_{\sigma}$ is replaced by its tensor product 


$$
J_{p}\left(\nabla u_{\delta}\right)=K_{p} *\left(\nabla u_{\delta} \otimes \nabla u_{\delta}\right)
$$

Structure tensor is equal to act two linear diffusions on $u$, one with small diffusivity and another with large diffusivity, and to make parameter of structure tensor to suit for the smoother and smoother image through the iterative process, it leads to following structure tensor.

$$
H_{l(t)}\left(\nabla u_{\delta(t)}\right)=G\left(\nabla u_{\delta(t)} \otimes \nabla u_{\delta(t)}\right)=\left(\begin{array}{l}
G\left(\left(u_{\delta(t), x}\right)^{2}\right), G\left(u_{\delta(t), x} u_{\delta(t), y}\right) \\
G\left(u_{\delta(t), x} u_{\delta(t), y}\right), G\left(\left(u_{\delta(t), y}\right)^{2}\right)
\end{array}\right)
$$

Eigenvalue $\lambda_{2}$ should be positive in the edge direction and eigenvalue $\lambda_{1}$ in the gradient direction should be positive in the flat region of image, but to sharpen edges, $\lambda_{1}$ should be negative in the edge region of image. $\lambda_{1}, \lambda_{2}$ are defined as

$$
\begin{aligned}
& \lambda_{1(x)}= \begin{cases}1-\left(x / k_{f}\right)^{n} & 0 \leq x \leq k_{f} \\
\beta\left[\left(\left(x-k_{b}\right) / w\right)^{2 m}-1\right] & k_{b}-w \leq x \leq k_{b}+w \\
0 & \text { else }\end{cases} \\
& \lambda_{2}=\alpha+(1-\alpha) \exp \left(-\frac{B}{\left(\mu_{1}-\mu_{2}\right)^{2}}\right)
\end{aligned}
$$

New diffusion model tensor $D^{*}$ is constructed by $p_{1}, p_{2}, \lambda_{1}, \lambda_{2}$ and following diffusion model is made:

$$
\begin{aligned}
& \partial_{t} u=\operatorname{div}\left[D^{*} \nabla u\right], \\
& D^{*}=\left(p_{1}, p_{2}\right)\left(\begin{array}{l}
\lambda_{1}, 0 \\
0, \lambda_{2}
\end{array}\right)\left(\begin{array}{l}
p_{1}^{T} \\
p_{2}^{T}
\end{array}\right)
\end{aligned}
$$

The operator $\nabla(\cdot)$ is calculated by 


$$
\left.\nabla u\right|_{i, j}=\frac{1}{2}\left(\begin{array}{l}
u_{i+1, j}-u_{i-1, j} \\
u_{i, j+1}-u_{i, j-1}
\end{array}\right)
$$

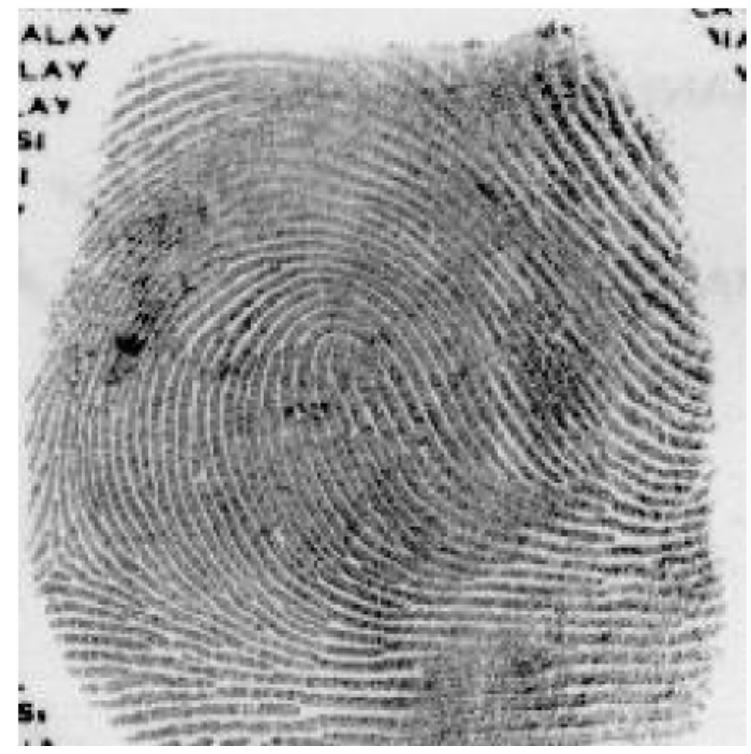

(a)

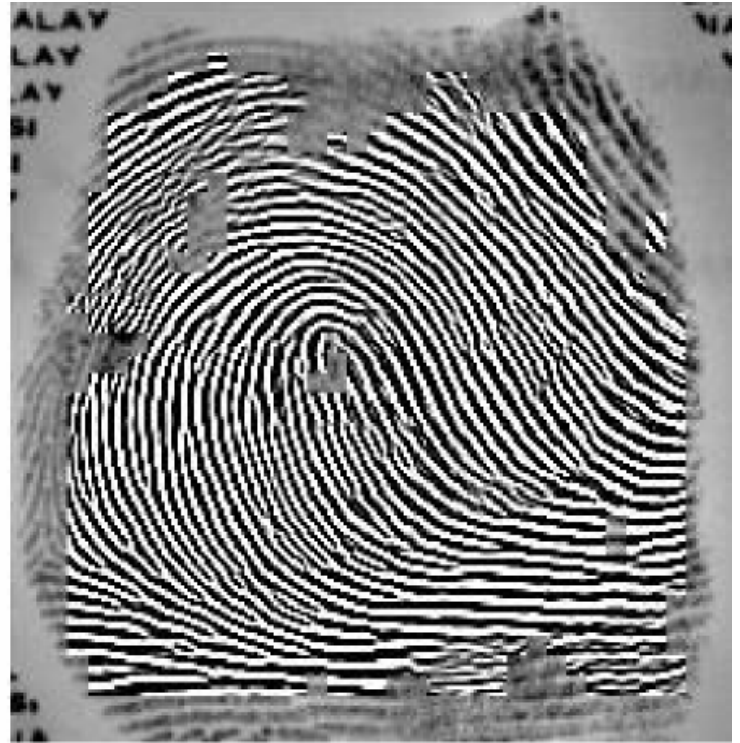

(b)

Figure 1.11: (a) Input fingerprint image, (b) Enhanced fingerprint image [16].

\subsection{Image Processing Techniques}

There is a number of image processing techniques for image enhancement and image restoration. The ultimate goal of enhancement and restoration is to improve an image in some predefined sense. Image enhancement is a subjective process whereas image restoration is for the most part an objective process [17]. Some of the enhancement and restoration techniques are mentioned in brief below: 
Blind Deconvolution: It is a process which helps in recovery of target object from set of blurred images in the presence or a poorly determined or unknown point spread function (PSF). The blind deconvolution approach allows constructing an algorithm which takes noisy image and noisy PSF as input and gives an estimate of both the PSF and unblurred image. Blind deconvolution applies to restoration of grey-scale images. There are various algorithms and implementations that exist for performing blind deconvolution.

Restoration and enhancement by spatial filtering: Spatial filtering is used when only additive noise is present in the image. There is a number of noise reduction spatial filters. The simplest of all is arithmetic mean filter. It computes the average value of the corrupted image $\mathrm{g}(\mathrm{x}, \mathrm{y})$ in the area defined by $\mathrm{S}_{\mathrm{xy}}$. The value of restored image at any point is simply the arithmetic mean computed using the pixels in the region defined by $\mathrm{S}_{\mathrm{xy}}$.

Median filter which is best known order-statistics filter replaces the value of pixel by the median of the grey levels in the region defined by $S_{x y}$. Median filter is effective in presence of both bipolar and unipolar impulse noise. Other orderstatistics filters are max and min filters, which as the name suggests, value at a point in restored image is maximum and minimum grey level of the region $\mathrm{S}_{\mathrm{xy}}$ respectively.

Restoration and enhancement by frequency domain filtering: Bandreject filtering is used for noise removal where the locations of the noise components in 
the frequency domain are known. It removes or attenuates a band of frequencies about the origin of the Fourier transform. The bandpass filter performs the opposite operation to bandreject filter. It generally removes too much detail from the image. However, bandpass filtering is quite useful in isolating the effect on an image of selected frequency bands [17]. A notch filter rejects frequencies in predefined neighborhoods about a center frequency. Due to symmetry of the Fourier transform, notch filters must appear in symmetric pairs about the origin in order to obtain meaningful results [17].

Minimum mean square error (Wiener) filtering: This technique assumes that if noise is present in the system, then it is considered to be additive white Gaussian noise (AWGN). Wiener filtering requires a priori knowledge of the power spectra of noise and the original image. A simplified equation of wiener filter is given below for 1D case [17]:

$R(u)=\frac{H(u)^{*}}{\mid H(u)^{2}+K}$

The Wiener filter behaves as a bandpass filter, where the highpass filter is due to inverse filter and lowpass filter is due to parameter $\mathrm{K}$.

\subsubsection{Image Registration}

Image registration is a task of finding a corresponding function which maps coordinates from a reference image to coordinates of homologous points in a test image $[18,19]$. Images may have been captured at different times, from different sensors, or from 
different view points. Image registration is a significant component in most of the image evaluating systems. Some specific examples are matching a target with a real time image of a scene for target recognition, monitoring global land usage using satellite images, and aligning images from different medical modalities for diagnosis.

There are many variations which can occur in images, and a number of registration techniques have been proposed in the literature. According to the survey on registration techniques by brown [19], three major types of variations are distinguished. First type of variations causes images to be misaligned due to difference in acquisition. To remove this misalignment a spatial transform is found. Second type of variation is not only due to difference in acquisition but also due to change in lighting and atmospheric conditions. This type of variation leads to change in intensity and may cause perspective distortions. Third type of variation is caused due to object moments, growth, change in scene when images are captured at different time.

There are four traditional methods mostly used for registration purpose: Correlation and Sequential based Methods, Fourier Methods, Point Mapping, Elastic Model-Based Matching. These methods are characterized by the complexity of their corresponding transformation class. Correlation and Sequential methods are applicable for small well defined affine transformations. Similar limitation is applied to Fourier methods. However, these methods can be more effective in the presence of frequency-dependent noise. When misalignment is no longer affine but more complex than that, then Point Mapping with a feedback can be used. Point mapping by interpolation performs well if it 
is possible to find accurate matches for control points; otherwise approximation is necessary for matching control points. If global transformations are not sufficient then local methods like piecewise interpolations can be used. However, if there are uncorrelated local variations additional knowledge such as elastic membrane for modeling possible image deformations is used.

Due to a great variety of methods available for image registration, question arises on selecting an appropriate method for a specific problem. Knowledge of the cause of distortions present in image to be registered should be used as much as possible in designing and selecting a method for a particular application [19]. The class of transformations which are determined by the type of distortion present in images, and its complexity determine the general type of method to be used.

The registration technique is specified by selecting a particular feature space, similarity metric, search space, and search strategy from the types of methods available for registration. Feature space extracts the intrinsic structures in the image and the irrelevant information is removed by reducing the search space. Similarity metric defines the test to be made for each possible match, for example cross-correlation is robust for white noise, similarity metrics based on the invariant properties of Fourier Transform are good candidates for frequency dependent noise. Knowledge available concerning the source of distortion and assumptions about the imaging system and scene properties can be exploited for search space and search strategy. 
Registration problem becomes very difficult when local variations are present. A feedback between feature detection, similarity measurements, and computing optimal transformations can be used to overcome this problem. When local distortions are present, iterations, cooperation and hierarchical structures can be used with global information to speed the registration process. When designing or choosing a technique for registration; distinction between global and local transformations and methods, global and local distortions, and global and local computations should be carefully considered [19].

In our case, we deal with a fingerprint image registration problem. Recognition system would give better performance if registered images are of good quality. We have proposed to enhance a fingerprint image using other images in the same class. Therefore, it is critical for the images to be aligned. Misalignment in case of fingerprint images is very complex. Therefore, apart from affine transform we use optical flow based registration.

\subsubsection{Image Inpainting}

Image inpainting is a technique which restores a damaged image by means of image interpolation [20]. Various applications of image inpainting include photo restoration, wireless image transmission (e.g. recovering lost blocks), zooming, image coding etc. Image inpainting fulfills the purpose of filling-in of missing information which is very important in applications stated before. Inpainting fills in the missing information by interpolation from the vicinity. Figure 1.12 shows some results for region filling. 


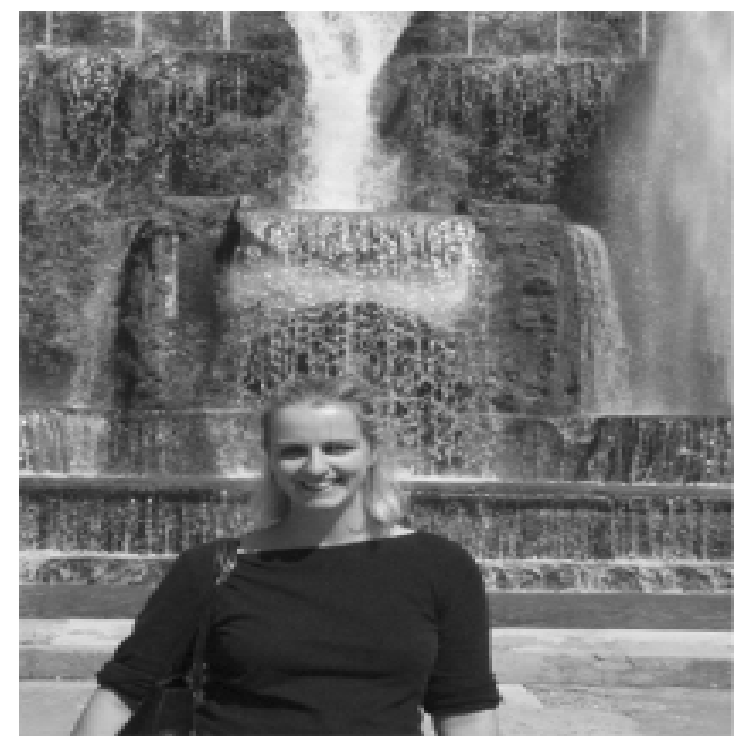

(a)

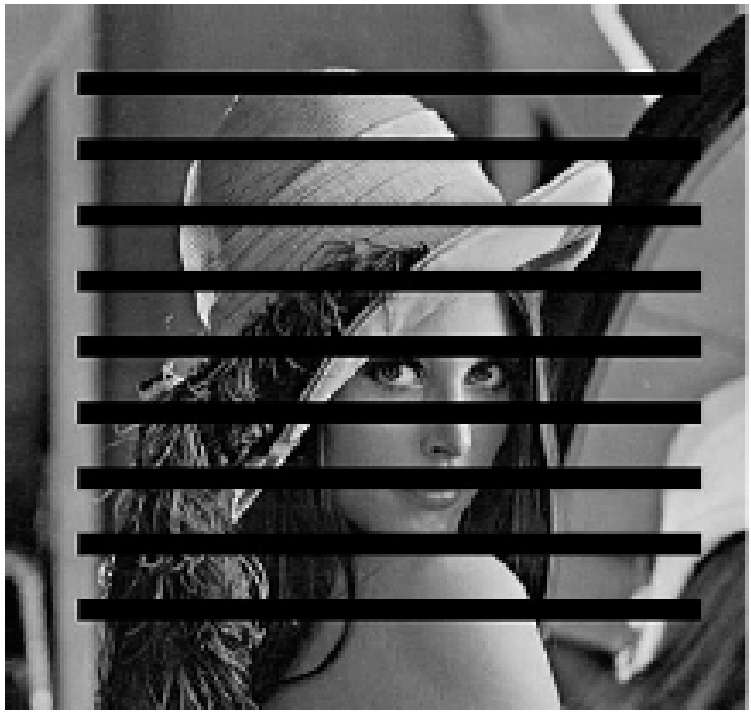

(c)

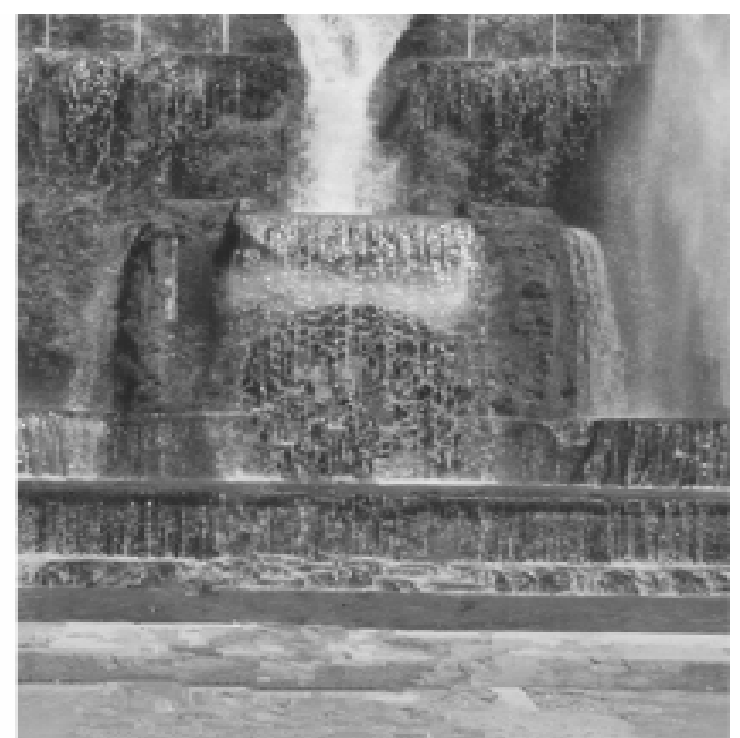

(b)

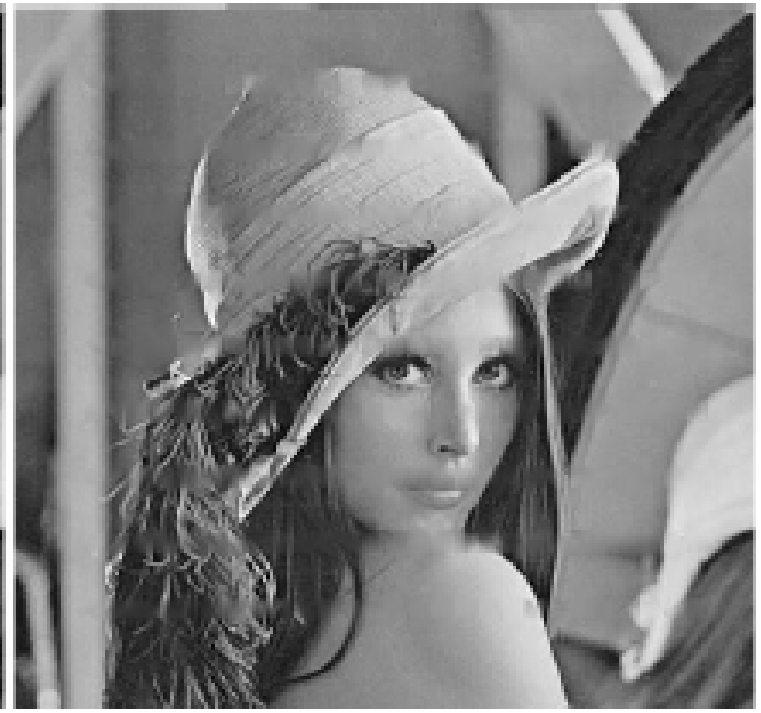

(d)

Figure 1.12: (a) Original image, (b) Object in the foreground is removed and the region is filled [21], (c)"Lena" image degraded with artificial artifacts , (d) Restored "Lena" [22]. 
There is a number of image inpainting techniques available in the literature. Some of them are mentioned in brief below.

"Fast Digital Image Inpainting" proposed by Oliveira et al. [23] considers $\Omega$ to be a small area to be inpainted and $\partial \Omega$ be its boundary. The area $\Omega$ is repeatedly convolved with a diffusion kernel and after a number of iterations inpainting progresses from $\partial \Omega$ to $\Omega$. This technique used weighted average kernel that only considers contributions from neighbor pixels.

Haber et al. [24] proposed "Image Restoration using Multiresolution Texture Synthesis and Image Inpainting". In this technique, user is provided with the binary mask that identifies image regions to be reconstructed. Input image is decomposed in high frequency and low frequency parts using DCT. Fast digital image inpainting proposed by Oliveira et al. [23] is applied to masked areas of low frequency parts. High frequency parts are decomposed using Gaussian pyramid and multiresolution texture synthesis is used in masked areas. Finally low and high frequency parts are summed up.

Criminisi et al. [21] proposed "Region Filling and Object Removal by Exemplar-Based Image Inpainting". A target region $\Omega$ to be removed and filled is selected and the rest of the area $\Phi$ in the image is the source region. Next, exemplar based texture synthesis [21] is used to fill the required region by selecting a template window $\psi$ of size $9 \times 9$ pixels. 
Peng et al. [25] introduced digital image inpainting by using Monte Carlo method. Inpainting is based on an improved Mumford-Shah image model:

$$
E[u, \tau]=\frac{\gamma}{2} \int_{\Omega / \tau}|\nabla u|^{2} d x+\int_{\tau}\left(\alpha\left|X^{\prime}(s)\right|^{2}+\beta\left|X^{\prime \prime}(s)\right|^{2}\right) d s
$$

The first function forces the image function to be harmonic on the smooth region $\Omega / \Gamma$. Random simulation of boundary integral to interpolate the smooth region of the inpainting domain is used. This is a Monte Carlo method. The second term is the elastic energy of the active contour model; it is used to connect the broken edges reaching the boundary of inpainting domain.

In this work, we propose to enhance local low quality regions in a fingerprint image. Image inpainting as explained above, fills in the missing information using interpolation technique which is same problem in our case. Hence, we propose to repair low-quality regions in a fingerprint image by using image inpainting based approach.

\subsection{Motivation}

Recognizing a person based on his physiological and behavioral characteristics is known as biometrics. Biometrics has gained a lot of importance in recent past due to increase in crime rate and need of more robust and automated security. People from many different backgrounds have contributed for development of biometric system for number of years, for example researchers from fields like sensors, image processing, signal processing, pattern recognition, database management, software development etc. Fingerprint is the most widely used biometric trait for purpose of authentication. 
Fingerprints from the same user can vary significantly due to different sensors used, different atmospheric conditions, different lighting conditions, different pressure applied by the user on the sensor and number of other factors. This motivated us to develop an enhancement algorithm for fingerprints which will help in image registration part. Image registration is a very important part of a biometric system because there can be many variations in image and no single technique can give good results for every class of variations mentioned in previous section.

This work concentrates on enhancement of fingerprint images prior to feeding them into a template generation module. Prior to enhancement first of all we detect the low quality regions in a fingerprint which are basically dry areas or wet areas. We perform fingerprint image quality analysis using coherence of direction and detect low quality regions which are presented locally in a fingerprint image.

For enhancement purpose we propose single-impression and multi-impression enhancements. In the case of a single copy, after the low quality areas are detected, ridge flow in those areas is estimated by using image inpainting. Inpainting is done by interpolation of bad areas from the vicinity.

For multi-impression, enhancement is based on the intuition that, the probability of the same local region to be of low quality in multiple fingerprints images of the same user is very low. But before enhancement, fingerprint images need to be aligned. For alignment, we perform the traditional affine transform with scaling and apply Horn-Schunck method 
which provides more robust alignment. Finally, we interpolate the low quality regions from the regions in other fingerprint image which are at the same location.

\subsection{Thesis Organization}

After the general introduction of biometric system, various traits, image inpainting and image registration, the next chapter deals with a background and related works in the area of fingerprint enhancement. Since, there is a number of fingerprint enhancement algorithm in the literature, the next chapter gives a brief overview of the most commonly used enhancement algorithms.

The third chapter deals with a single copy enhancement. It is divided into two parts. First part deals with the detection of low quality areas by coherence map. After the coherence map is generated, a suitable threshold is applied on coherence values and low quality areas are detected. The second part gives details of image inpainting technique used for interpolation on low quality areas from the vicinity. After detection of low quality areas, a binary mask is generated and interpolation is done in masked areas from the neighborhood.

The fourth chapter deals with a multiple copy enhancement. In case of multiple copy enhancement, a low quality fingerprint is enhanced by referring a good quality image of the same class. Before enhancement these two fingerprints should be aligned as precise as possible, for which an affine transform and Horn-Schunck method for finding optical flow are applied for alignment. Similarly, to a single copy enhancement, a binary mask is generated for low quality fingerprint and the masked areas are interpolated from the good 
quality regions in the other fingerprints of good quality. Fifth chapter gives the results of enhancement followed by the conclusion and the future work in sixth chapter. 


\section{Chapter 2: Fingerprint Quality Assessment}

For a particular biometric to be effective each individual should have the biometrics and it should be easy to sample or acquire. In real time, adverse signal acquisition conditions and inconsistent presentation of signal often result in unusable or nearly unusable biometrics signals or samples [10]. Most significant role in poor accuracy performance of a biometric system is played by the poor quality of the actual machine sample of a biometrics.

Most important reason for high false accept/reject rates in a deployed system is the poor acquisition of biometric samples. There are two solutions to this: one can either probabilistically model and weigh all the adverse situations into the feature extraction/matching stage, or one can try to dynamically and interactively obtain a desirable input sample [10].

Fingerprints are well known for their distinctiveness among individuals. Even twins do not have same fingerprints. Fingerprints are characterized by ridges and valleys, where ridges are the dark and valleys are bright. It is due to ridge pattern and local ridge characteristics that uniqueness of fingerprints is determined. During fingerprint image acquisition there are some challenging aspects because of contact problems.

1. Nonuniform contact: Due to various reasons all ridges are not in complete optical contact with the glass platen, due to which all ridge structure is not completely captured. 
2. Inconsistent contact: 3D shape of the finger is mapped onto the $2 \mathrm{D}$ surface, which is uncontrolled and results in inconsistent mapped regions across impressions. This is caused due to different pressure of the different areas of the finger on the imaging surface.

3. Irreproducible contact: Sometimes due to accidents, manual work etc. there is permanent or semipermanent change in the ridge structure, which leads to the spurious minutiae or minutiae like features.

4. Sometimes there are residues left over from the previous fingerprint capture, which adds the noise to the image.

5. Due to imperfect imaging conditions, imaging system distorts the image of the sensed finger.

Sometimes due to skin conditions (e.g., wet, dry, cuts etc.), the ridges in some areas on the fingerprint image are not clearly visible. Due to these skin conditions, contact problems and many other factors, a significant percentage of fingerprints are of low quality. Figure 2.1 shows examples of fingerprints with dry areas, scars, and wet areas.

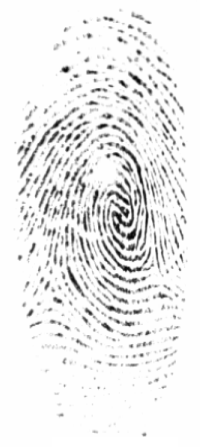

(a)

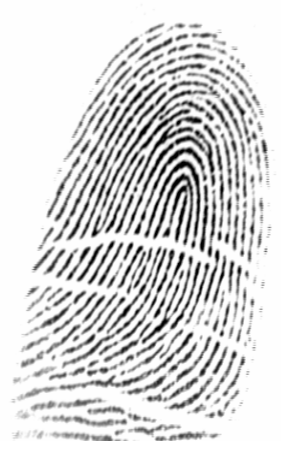

(b)

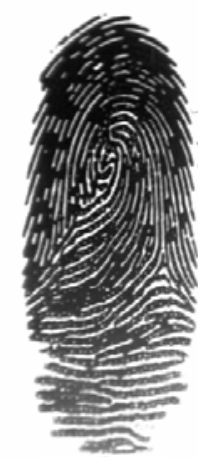

(c)

Figure 2.1: Fingerprint image (a) with dry areas, (b) with scars, (c) wet areas 
In this chapter we propose to detect low quality regions (wet and dry) in a fingerprint image with the use of direction based method which is mainly based on coherence of direction.

\subsection{Coherence of direction}

Coherence is the measure for the local strength of the directional field. Directional field describes the orientation of the ridge valley structure and in principle; it is perpendicular to the gradients which are orientations at pixel scale [33]. Coherence basically, indicates how well gradients are pointing in the same direction. Figure 2.2 shows the gradients in part of a fingerprint and figure 2.3 shows the averaged directional field.

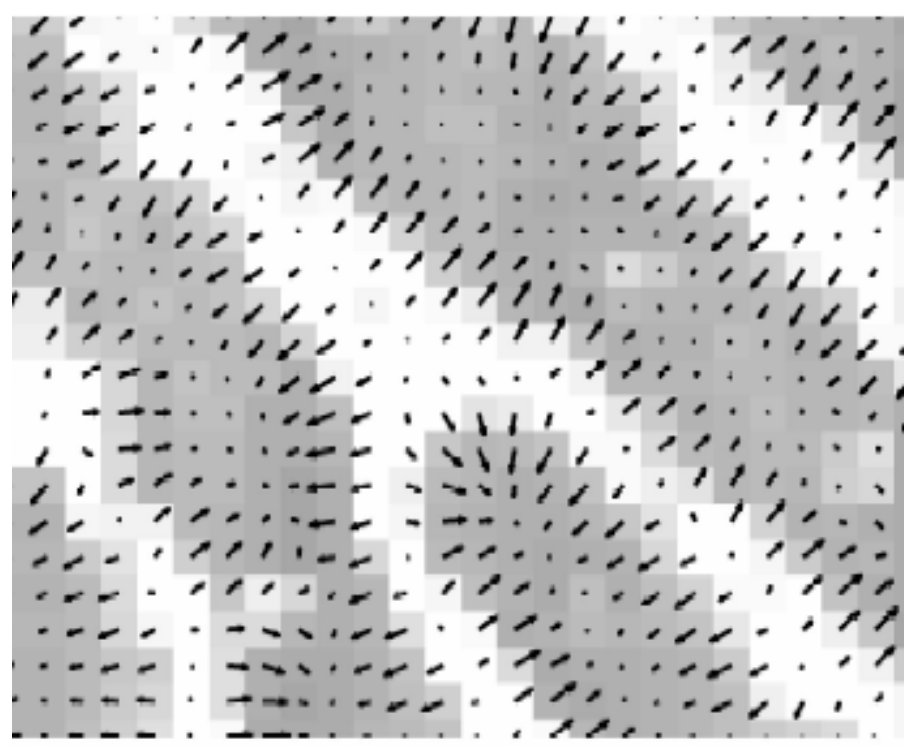

Figure 2.2: Gradients in part of a fingerprint [33] 


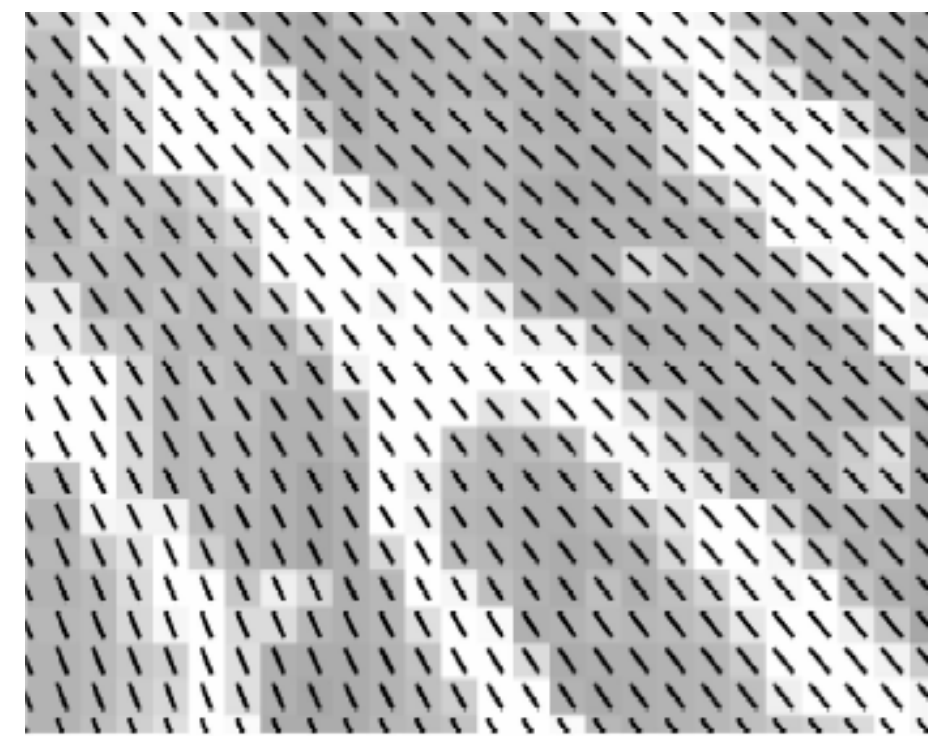

Figure 2.3: The averaged directional field in part of a fingerprint [33]

\subsection{Low quality region detection}

Before the detection of low quality regions, segmentation of fingerprint image is done and then low quality regions are detected in the segmented fingerprint. Segmentation is based on the local variance of gray level is used to locate the region of interest from the fingerprint image. Fingerprint image is divided into blocks of size 16 x 16 and the variance of gray levels in a direction orthogonal to the orientation field in each block is computed. The assumption is that the background has low variance in all the directions [34]. Figure 2.4 (b) shows the variance field of the original image 2.4 (a). The segmented image is shown in Figure $2.4(\mathrm{~d})$. 


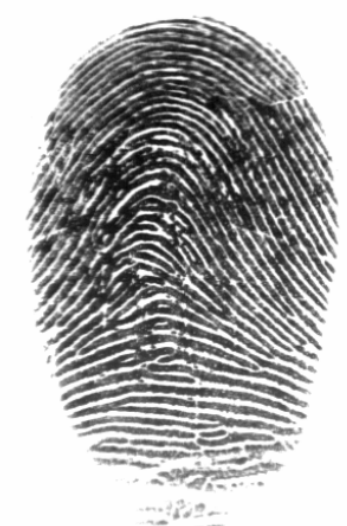

(a)

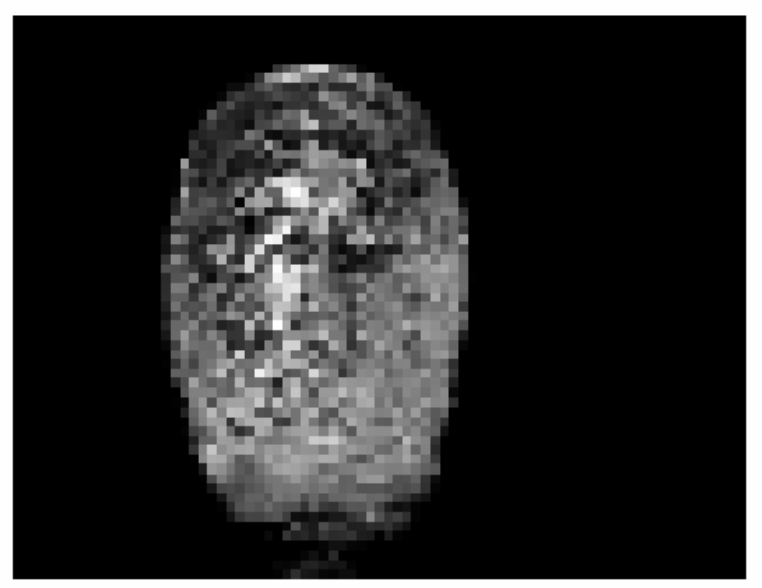

(b)

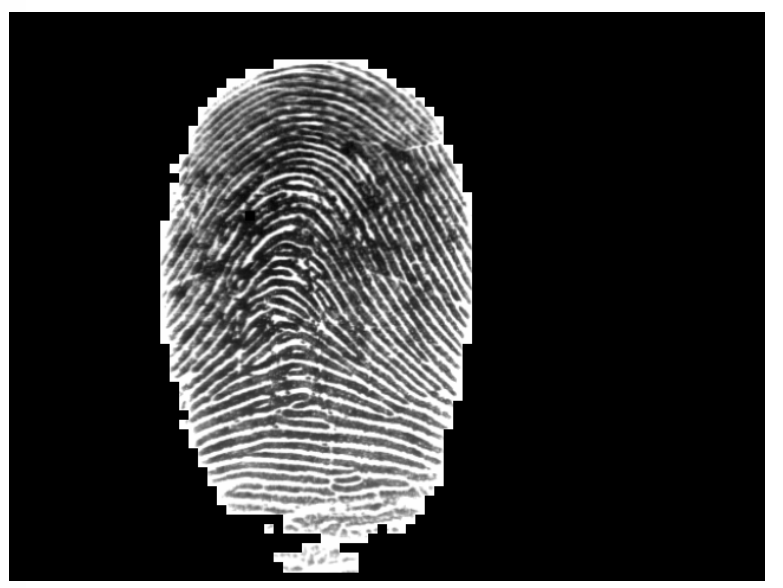

(c)

Figure 2.4: (a) Original fingerprint image, (b) Variance field of the fingerprint image, (c) Segmented fingerprint image.

Coherence has been mainly used for segmentation of fingerprint images. Since a fingerprint mainly consists of parallel line structure, the coherence will be higher in the foreground than in the background [35]. Same concept can be applied for detection of low quality regions in a fingerprint. Since, low quality regions are presented locally in the fingerprint therefore, coherence is computed locally and it will be significantly smaller in 
the regions which are wet or dry as the parallel line structure of the ridges in these areas will not be there.

Let in an image $I, I(x, y)$ represent the intensity of pixel at $(x, y)$, and $G_{x}(x, y)$ and $G_{y}(x$, $y)$ denote the gradients at the pixel $(\mathrm{x}, \mathrm{y})$, then the coherence in a window center at $(\mathrm{x}, \mathrm{y})$ can be computed using the following equation:

$$
\begin{aligned}
& O_{x}(i, j)=\sum_{u=i-\frac{w}{2}}^{u=i+\frac{w}{2}} \sum_{v=j-\frac{w}{2}}^{j+\frac{w}{2}} 2 G_{x}(u, v) G_{y}(u, v) \\
& O_{y}(i, j)=\sum_{u=i-\frac{w}{2}}^{u=i+\frac{w}{2}} \sum_{v=j-\frac{w}{2}}^{j+\frac{w}{2}}\left(G_{x}^{2}(u, v)-G_{y}^{2}(u, v)\right) \\
& O_{E}(i, j)=\sum_{u=i-\frac{w}{2}}^{u=i+\frac{w}{2}} \sum_{v=j-\frac{w}{2}}^{j+\frac{w}{2}}\left(G_{x}(u, v)+G_{y}(u, v)\right)^{2} \\
& \text { Coh }=\sqrt{\frac{O_{x}^{2}(i, j)+O_{y}^{2}(i, j)}{O_{E}(i, j) * w * w}}
\end{aligned}
$$

For segmentation purpose, if Coh is bigger than a threshold, the block is considered as foreground otherwise background. Similarly, if Coh is bigger than a certain threshold than it is considered as a good quality region otherwise it is considered as a region of low quality. 
There are many other fingerprint quality assessment techniques as described in previous chapters. These techniques makes the use of global as well as local features of a fingerprint image but non of them can be used to detect low quality regions in a fingerprint image. Low quality regions are present locally in a fingerprint image, so detection should be based on local features. Fingerprint quality assessment methods like proposed by Aguilar et al. which based on the the energy of the power spectrum cannot be used because it assesses qualuty of a fingerprint as whole. It tells whether a fingerprint is of good quality or poor quality. It gives no idea about which regions are of poor quality.

Our method is based on coheence of direction, as in the local regions which are either wet, dry or have scars coherence will be considerably lower as compared to the regions of good quality where ridge and valleys are clearly visible. 


\section{Chapter 3: Fingerprint Enhancement: Single Impression}

Fingerprints are well known for their uniqueness among individuals and this uniquesness is determined by the several ridge characteristics and their relationship. Out of more than 100 ridge charactristics, two most prominent local ridge characteristics used for automatic fingerprint identification are ridge endings and ridge bifurcations (called minutiae). Where a ridge ends abruptly, that location is known as ridge ending. Where ridge forks or diverge into branch ridges, that location is known as ridge bifurcation.

Currently minutiae based fingerprint matching algorithm are being used in most widely used automatic fingerprint identification techniques. Hence, reliable extraction of minutiae is very important for good performance of identification technique. Extraction of minutiae depends significantly on quality of the fingerprint image. In ideal case ridges and valleys are clearly visible and ridges can be easily detected and minutiae can be precisely located from a binary ridge map. However, in practice due to variation in impression conditions, ridge configuration, skin conditions, acquisition device and noncooperative attitude of subjects, etc., a significant percentage of acquired fingerprint images is of poor quality [6]. This can significantly affect the performance of fingerprint identification system.

To ensure the perfomrance of minutiae extraction algorithms are robust with respect to the quality of fingerprint image, a fingeprint enhancement algorithm which can improve the quality of fingerprint image, is thus necessary. The goal of fingerprint enahancement 
algorithm is to improve the ridge structure in the input fingerprint image to facilitate the extraction of ridges and minutiae, on the other hand it should not create spurious ridge structures, as it may change the individuality of a fingerprint image.

In this chapter we introduce a fingerprint enhancement technique based on least square based image inpainting technique. After, the detection of the low quality regions as explained in the previous chapter, ridge structure in these regions is improved by using least square based image inpainting technique. This technique interpolates image pixels in the low quality regions fron their vicinity.

\subsection{Image Inpainting}

Image inpainting is a technique which restores damaged image by means of image interpolation [20]. Various applications of image inpainting include photo restoration, wireless image transmission (e.g. recovering lost blocks), zooming, image coding etc. Image inpainting fulfills the purpose of filling-in of missing information which is very important in applications sated before. Inpainting fills in the missing information by interpolation from the vicinity.

We use "Novel Sequential Error-Concealment Techniques Using Orientation Adaptive Interpolation" proposed by Xin $\mathrm{Li}$ and Michael $\mathrm{T}$. Orchard [22], to interpolate low quality regions in a fingerprint after they have been detected as explained in previous chapter. This technique is discussed in detail below: 
Instead of simultaneously recovering the pixels in a block author proposed to recover them in sequential fashion such that previously recovered pixels can be used in the recovery process afterwards. This technique basically transforms the original MAP estimation problem which requires a blockwise conditional probabilistic model into a series of easier MAP estimation problem that only require a pixelwise conditional probabilistic model [22]. In case of parallel recovery reconstructed image might suffer from edge blurring or various artifacts (e.g. , false edge) but in case of sequential recovery it has improved capability of modeling important image features like edges as it can infer important edge features from local available information. As previously recovered pixels are also used to resolve the uncertainty of missing pixels in addition to successfully recovered pixels, this technique suffers from the error propagation problem. As a solution to this problem, authors proposed to recover the corrupted block from eight different scanning orientations and take their linear merge as the final result.

This sequential structure has an improved capability of recovering important image features such as edges, especially in the two scenarios: 1) when there are more than one edge in the missing block, which is true in our case of fingerprint images and 2) when the block loss in consecutive, this can also be true in case of fingerprint images. In these scenarios, parallel approaches using blockwise prior models often can not reliably estimate the quantity and orientation of the edge feature, reconstructed images would suffer from blurred edges or annoying artifacts. In contrast, sequential recovery offers the flexibility of using local available information to adapt the recovery process such that important edge features are well preserved in the reconstructed image. 
Within the framework of sequential recovery, authors proposed a novel orientationadaptive interpolation scheme to solve the individual MAP estimation problem i.e., maximize $p\left(X_{k} \mid X_{1}, X_{2}, \ldots \ldots \ldots \ldots \ldots X_{k-1}, Y_{1}, Y_{2}, \ldots \ldots \ldots \ldots, Y_{L}\right)$.

\subsubsection{Orientation Adaptive Interpolation}

The available neighbors of a corrupted pixel $X_{n}$ could be either successfully received pixels $Y_{l}(l=1,2, \ldots \ldots \ldots \ldots, L)$ or previously recovered pixels $X_{k}(k=1,2, \ldots \ldots \ldots \ldots, n-1) . Y_{l}$ and $X_{k}$ are not distinguished anymore, instead the set $M_{n}=$ $\left\{X_{n-1}, X_{n-2}, \ldots \ldots \ldots \ldots . . ., X_{1}, X_{0}=Y_{1}, Y_{2}, \ldots \ldots \ldots . ., X_{n-M}=Y_{L}\right\}, M=n+L-1$ is used, to denote all the neighbors of $X_{n}$. The following two assumptions that reasonably hold for image source are made:

1) Although globally image source cannot be modeled by a stationary Gaussian process, it can be viewed as local stationary. Therefore, the conditional probability distribution function $p\left(X_{n} \mid M_{n}\right)$ is mostly characterized by the second-order statistics (covariance).

2) The image source satisfies the Nth-order Markov property, i.e., $p\left(X_{n} \mid X_{n-1}, X_{n-2}, \ldots \ldots \ldots \ldots.\right)=p\left(X_{n} \mid X_{n-1}, X_{n-2}, \ldots \ldots \ldots \ldots X_{n-N}\right)$. 
Under these assumptions, MAP estimation problem becomes the linear minimum meansquare error (MMSE) estimation problem of minimizing $E\left\{\left(X_{n}-\hat{X}_{n}\right)^{2}\right\}$ where $\hat{X}_{n}$ the linear estimation of is $X_{n}$ based on $N_{n}=\left\{X_{n-1}, X_{n-2}, \ldots \ldots \ldots X_{n-N}\right\}$.

$$
X_{n}=\sum_{k=1}^{N} a_{k} X_{n-k}
$$

According to the classical Wiener filtering theory [ref] [ref], the optimal coefficients $\vec{a}=\left[a_{1}, a_{2}, \ldots \ldots a_{N}\right]^{T}$ are only determined by the second-order statistics covariance of the Gaussian process

$\vec{a}=\left(R_{X X}\right)^{-1} \vec{r}_{X}$

where $\quad \vec{r}_{X}=\left[r_{1} \ldots . . r_{k} \ldots . . r_{N}\right], \quad r_{k}=\operatorname{Cov}\left\{X_{n} X_{n-k}\right\} \quad(1 \leq k \leq N) \quad$ and $\quad R_{X X}=\left[R_{k l}\right]$, $R_{k l}=\operatorname{Cov}\left\{X_{n-k} X_{n-l}\right\} \quad(1 \leq k, l \leq N)$. Equation 3.2 is the projection of $X_{n}$ onto the subspace spanned by $X_{n-1}, X_{n-2}, \ldots \ldots \ldots . . X_{n-N}$ in the least square sense. Due to the assumption \# 1 i.e., local stationary process covariance can be estimated inside the local window using a conventional "covariance method" [22, 52].

$$
\overrightarrow{R_{X X}}=C^{T} C, \quad \overrightarrow{\vec{r}}_{X}=C^{T} \vec{y}
$$

where $\vec{y}=\left[X_{n-1}, X_{n-2}, \ldots \ldots, X_{n-M}\right]^{T}$ is an $M \times 1$ vector containing all the elements inside the local window $M_{n}$ and

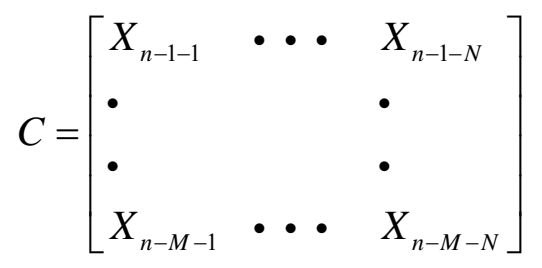


is an $M \times N$ matrix whose $k$ th row consists of the $N$ neighbors used to estimate $X_{n-k}$. By plugging equation 3.3 into 3.2 , we obtain $\vec{a}=\left(C^{T} C\right)^{-1}\left(C^{T} \vec{y}\right)$.

Such covariance-based adaptation is capable of tuning the support of $\vec{a}$ to get aligned along the arbitrary edge orientation $[22,53]$. Equation 3.5 preserves the important edge features than naïve linear interpolation techniques that assign the fixed interpolation coefficients uniformly for the whole image. Figure 3.1 shows the definition of $M_{n}$ and $N_{n}$.

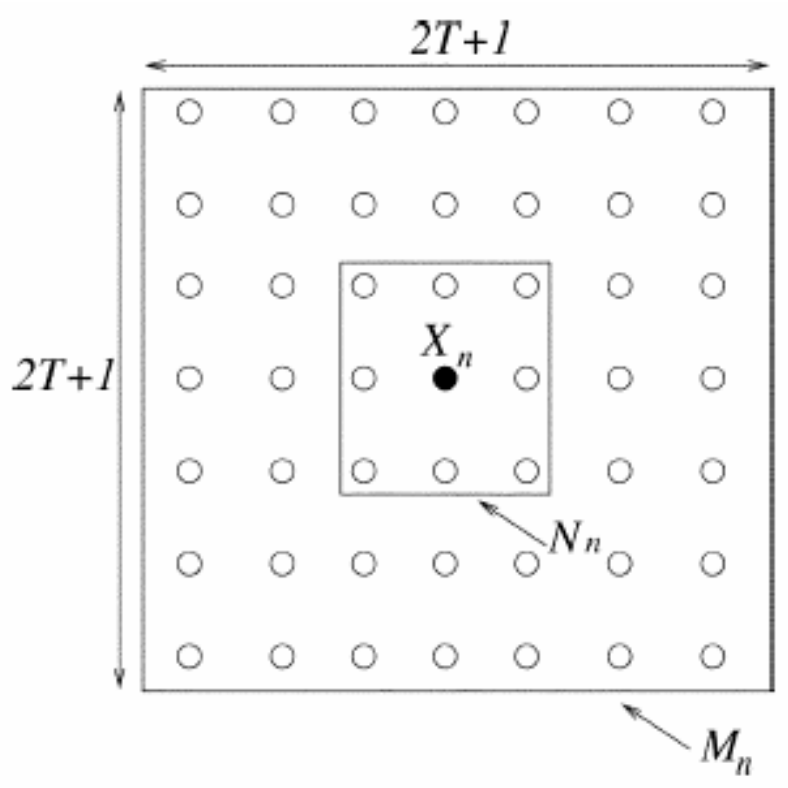

Figure 3.1: Definition of $M_{n}$ and $N_{n}$.

Summarizing the interpolation scheme, first the set of $M_{n}$ and $N_{n}$ are picked according to the definition and data matrix $C$ and data vector $\vec{y}$ are formulated. Now, the interpolation coefficients $\vec{a}$ are derived using equation 3.4 and they are substituted into 3.1 to obtain the interpolation result. As used the original proposed scheme, we also 
define $N_{n}$ to be the eight nearest neighbors of $X_{n}$ and the set $M_{n}$ to be the $(2 T+1)^{2}-1$ neighbors of $X_{n}$, within a squared window sized by $(2 T+1) \times(2 T+1)$ and centered at $X_{n}$ as shown in figure 3.1. If an element in the set $N_{n}$ is either successfully received or previously recovered, then it is said to be valid and all valid elements form the set $N_{n}^{*}$. All valid elements in the set $M_{n}$ form the set $M_{n}^{*}$, which are considered valid if the elements contained in its valid set $N_{n}^{*}$ are valid.

\subsection{Single impression enhancement}

Following are the steps proposed by us for the single impression fingerprint image enhancement:

1) First of all low quality regions (wet areas, dry areas, areas with scars etc.) are detected in a fingerprint image using coherence of direction as explained in the previous chapter.

2) A binary mask is generated with 0 as the low quality pixels and rest as 1 , as shown in Figure 3.2.

3) Using this binary mask "Sequential Error-Concealment Techniques Using Orientation Adaptive Interpolation" as explained before is applied on the fingerprint image to interpolate the masked areas which are basically low quality regions.

Results of enhancement are shown in chapter 5. 


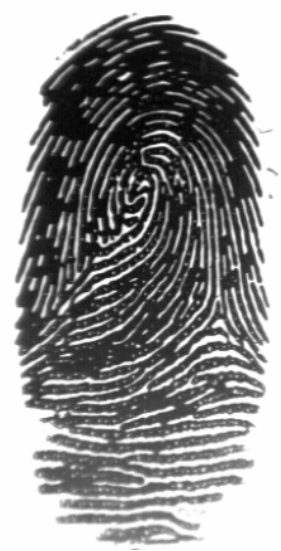

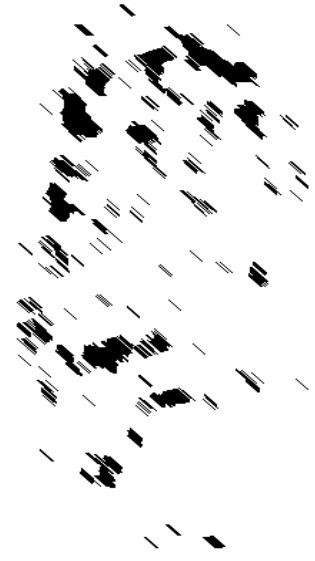

(b) (a)

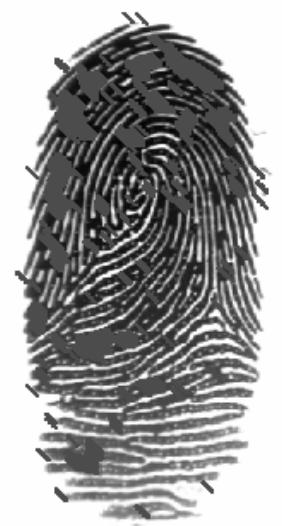

(c)

Figure 3.2: (a) Original fingerprint image with wet areas; (b) Binary mask with low quality regions as 0; (c) Fingerprint image with masked low quality regions 


\section{Chapter 4: Fingerprint Enhancement: Multiple Impression}

In this chapter we propose to enhance a fingerprint image i.e., to estimate the ridge flow in low quality areas (wet, dry, scars etc), using another fingerprint of good quality of the same class. It is based on the intuition that, the probability of same local region to be of low quality in multiple fingerprints images of a same user is very low. Hence, we propose to enhance the low quality areas of a fingerprint image by interpolating it using "Sequential Error-Concealment Techniques Using Orientation Adaptive Interpolation", explained in previous chapter, from another fingerprint image of the same class.

Before the interpolation is applied, it is obviously necessary that both the fingerprints images must be aligned with each other. Two fingerprints may be translated, rotated or scaled. There may be non-linear elastic deformation present in the fingerprints, which is very challenging problem. We do not take into account the non-linear elastic deformation in this work, due to its complexity. We try to align two fingerprint images using affine transformation and after that Horn-Schunck method of finding optical flow between two impressions is applied, which is explained later in the chapter. After alignment low quality regions are detected using coherence of direction explained in chapter 3 and a binary mask is generated which masks the detected low quality regions in a fingerprint image. Masked low quality regions are interpolated using "Sequential Error-Concealment Techniques Using Orientation Adaptive Interpolation", [22], not from the vicinity, instead, from the good quality regions which are located at the same position in the 
aligned fingerprint image, as the vicinity of masked low quality regions in the fingerprint image to be enhanced.

\subsection{Fingerprint alignment}

For fingerprint alignment first of all minutiae are extracted from each fingerprint image and the minutiae correspondence is found out. Minutiae extraction and correspondence are estimated using Verifinger SDK. There are number of attributes to describe minutiae, including its location in the fingerprint image, orientation, type (e.g., ridge termination or ridge bifurcation), a weight based on the quality of the fingerprint image in the neighborhood of the minutiae, and so on [3]. Most common attribute used and what given by verifinger SDK is that, each minutiae is considered as a triplet $\mathbf{m}=\{x, y, \theta\}$ that indicates the $\mathrm{x}, \mathrm{y}$ minutia location coordinates and the minutiae angle $\theta$.

$$
\begin{array}{ll}
F_{1}=\left\{m_{1}, m_{2}, \ldots \ldots, m_{m}\right\}, & m_{i}=\left\{x_{i}, y_{i}, \theta_{i}\right\}, \quad i=1 \ldots m, \\
F_{2}=\left\{m_{1}^{\prime}, m_{2}^{\prime}, \ldots \ldots, m_{n}^{\prime}\right\}, & m_{j}^{\prime}=\left\{x_{j}^{\prime}, y_{j}^{\prime}, \theta_{j}^{\prime}\right\}, \quad j=1 \ldots n
\end{array}
$$

Minutiae in two fingerprints are corresponding to each other, if the spatial distance (sd) between minutia $m_{j}^{\prime}$ in $F_{2}$ and minutia $m_{i}$ in $F_{1}$ is smaller than a given tolerance $r_{0}$ and the direction difference $(d d)$ between them is smaller than an angular tolerance $\theta_{0}$.

$$
\begin{aligned}
& s d\left(m_{j}^{\prime}, m_{i}\right)=\sqrt{\left(x_{j}^{\prime}-x_{i}\right)^{2}+\left(y_{j}^{\prime}-y_{i}\right)^{2}} \quad \leq r_{0} \quad \text { and } \\
& d d\left(m_{j}^{\prime}, m_{i}\right)=\min \left(\left|\theta_{j}^{\prime}-\theta_{i}\right|, 360^{0}-\left|\theta_{j}^{\prime}-\theta_{i}\right|\right) \quad \leq \theta_{0}
\end{aligned}
$$

After minutiae correspondence is determined fingerprints are aligned rigidly using affine transformation parameters of translation and rotation. Translation parameters are simply 
the $\mathrm{x}$-displacement and $\mathrm{y}$-displacement $[\Delta x, \Delta y]$, and let $\theta$ be the counterclockwise rotation around the origin. Affine transformation is given by:

$\operatorname{map}_{\Delta x, \Delta y, \theta}\left(m_{j}^{\prime}=\left\{x_{j}^{\prime}, y_{j}^{\prime}, \theta_{j}^{\prime}\right\}\right)=\left(m_{j}^{\prime \prime}=\left\{x_{j}^{\prime \prime}, y_{j}^{\prime \prime}, \theta_{j}^{\prime}+\theta\right\}\right)$, where

$\left[\begin{array}{l}x_{j}^{\prime \prime} \\ y_{j}^{\prime \prime}\end{array}\right]=\left[\begin{array}{ll}\cos \theta & -\sin \theta \\ \sin \theta & \cos \theta\end{array}\right]\left[\begin{array}{l}x_{j}^{\prime} \\ y_{j}^{\prime}\end{array}\right]+\left[\begin{array}{l}\Delta x \\ \Delta y\end{array}\right]$

After the two fingerprint images are aligned using the above explained affine transformation, they are not perfectly aligned with each other due to non-linear elastic deformation present in the fingerprint images. We propose to use Horn-Schunck method for determining optical flow between two impressions to align the images further. It is explained in the section below.

\subsubsection{Horn-Schunck method}

Optical flow is the distribution of apparent velocities of movement of brightness patterns in an image [54]. It is an approximation of the local image motion based upon local derivatives in a given sequence of images. Optical flow can be described in both 2D and 3D. In 2D it specifies the motion of image pixels between adjacent images while in 3D it specifies how much each volume voxel moves between adjacent volumes [55]. The computation of differential optical flow is basically a two step procedure [55]:

1. measure of spatio-temporal intensity derivatives (which is equal to measuring the velocities normal to the local intensity structures ) and 
2. Integrate normal velocities into full velocities, for example, either locally via least square estimation $[55,54,56]$ or globally via regularization $[55,56,57]$.

In case of fingerprint images we have to deal 2D optical flow. The basis of optical flow is the motion constraint equation which is explained below in 2D.

The 2D Motion Constraint Equation: Assume $I(x, y, t)$ is pixel inside a $n \times n$ neighborhood and in time $\delta t$ it moves by $\delta x$ in $\mathrm{x}$-direction and $\delta y$ in y-direction to $I(x+\delta x, y+\delta y, t+\delta t) \quad$ as shown in figure 4.1. Since $I(x, y, t)$ and $I(x+\delta x, y+\delta y, t+\delta t)$ are images of a same point, we have:

$I(x, y, t)=I(x+\delta x, y+\delta y, t+\delta t)$
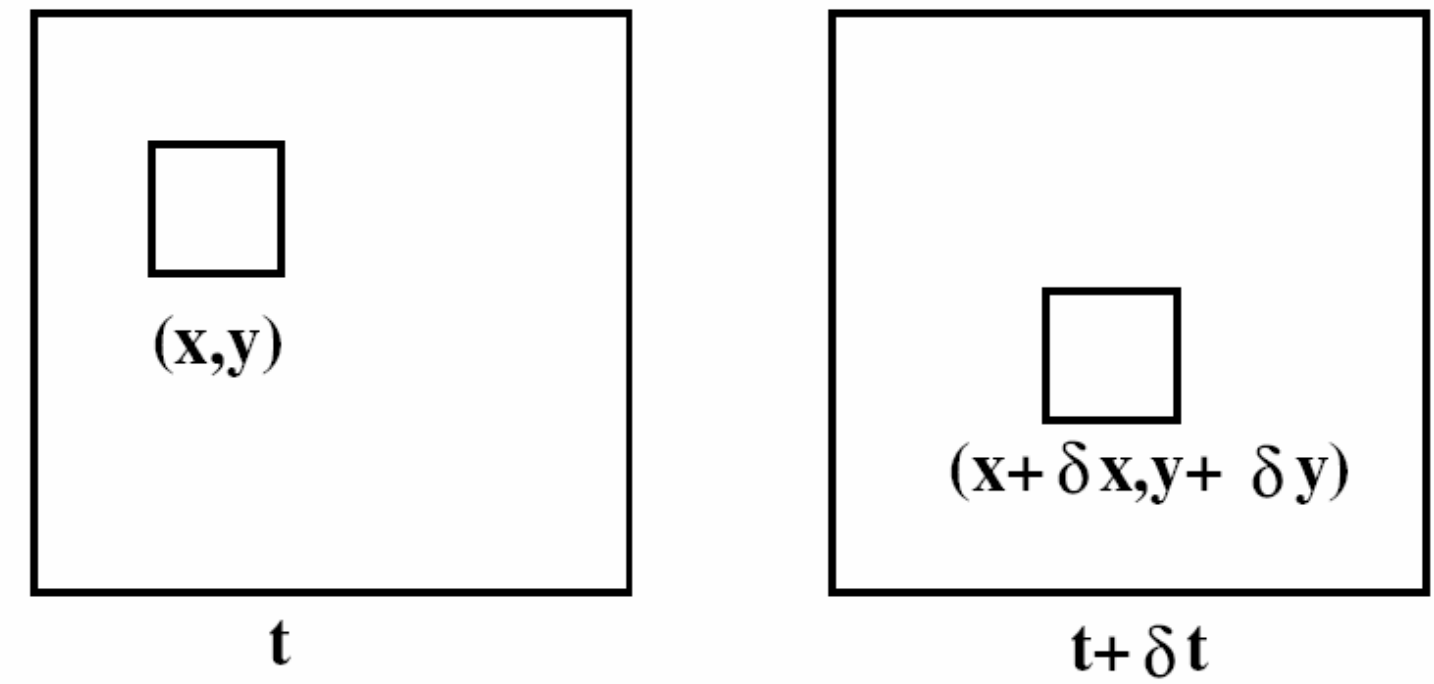

Figure 4.1: The image at position $(x, y, t)$ is same as at $(x+\delta x, y+\delta y, t+\delta t)$ [55].

The assumption made above is true for small local constraints provided $\delta x, \delta y$ and $\delta t$ are not too big. $1^{\text {st }}$ Order Taylor series expansion can be performed about $I(x, y, t)$ in equation 4.5 to obtain: 
$I(x+\delta x, y+\delta y, t+\delta t)=I(x, y, t)+\frac{\partial I}{\partial x} \delta x+\frac{\partial I}{\partial y} \delta y+\frac{\partial I}{\partial t} \delta t+H . O . T$

where, H.O.T are higher order terms which are assumed small can easily be ignored. Using 4.5 and 4.6 we obtain:

$\frac{\partial I}{\partial x} \delta x+\frac{\partial I}{\partial y} \delta y+\frac{\partial I}{\partial t} \delta t=0 \quad$ or

$\frac{\partial I}{\partial x} \frac{\delta x}{\delta t}+\frac{\partial I}{\partial y} \frac{\delta y}{\delta t}+\frac{\partial I}{\partial t} \frac{\delta t}{\delta t}=0$ and finally:

$\frac{\partial I}{\partial x} v_{x}+\frac{\partial I}{\partial y} v_{y}+\frac{\partial I}{\partial t}=0$

Here $v_{x}=\frac{\delta x}{\delta t}$ and $v_{y}=\frac{\delta y}{\delta t}$ are the $\mathrm{x}$ and $\mathrm{y}$ component of image velocity or optical flow and $\frac{\partial I}{\partial x}, \frac{\partial I}{\partial y}$ and $\frac{\partial I}{\partial t}$ are the image intensity derivatives at $(x, y, t)$. Normally, these partial derivatives are written as:

$I_{x}=\frac{\partial I}{\partial x}, \quad I_{y}=\frac{\partial I}{\partial y}$ and $I_{t}=\frac{\partial I}{\partial t}$

More compactly equation can be written as:

$\left(I_{x}, I_{y}\right) \cdot\left(v_{x}, v_{y}\right)=-I_{t}$ or as:

$\nabla I \cdot \vec{v}=-I_{t}$,

where $\nabla I=\left(I_{x}, I_{y}\right)$ is the spatial intensity gradient and $\vec{v}=\left(v_{x}, v_{y}\right)$ is the image velocity or optical flow at pixel $(x, y)$ at time t. $\nabla I \cdot \vec{v}=-I_{t}$ is called the 2D Motion Constraint Equation and it represents a line as shown in figure 4.2. This is due to aperture problem: there is usually insufficient local image intensity structure to measure 
full image velocity, but sufficient structure to measure the component normal to the local intensity structure [55]. Figure 4.2 (a) shows an example where a line up and to the right is viewed through a circular aperture. So, it is impossible to recover correct full image velocity but only the image velocity normal to the line. The problem of computing full image velocity then becomes finding an additional constraint that yields a second different equation in the same unknowns.

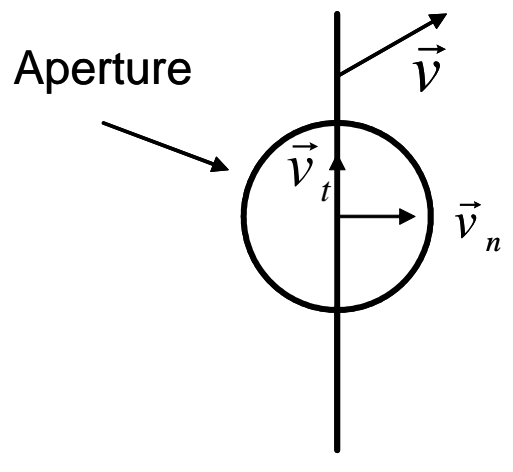

(a)

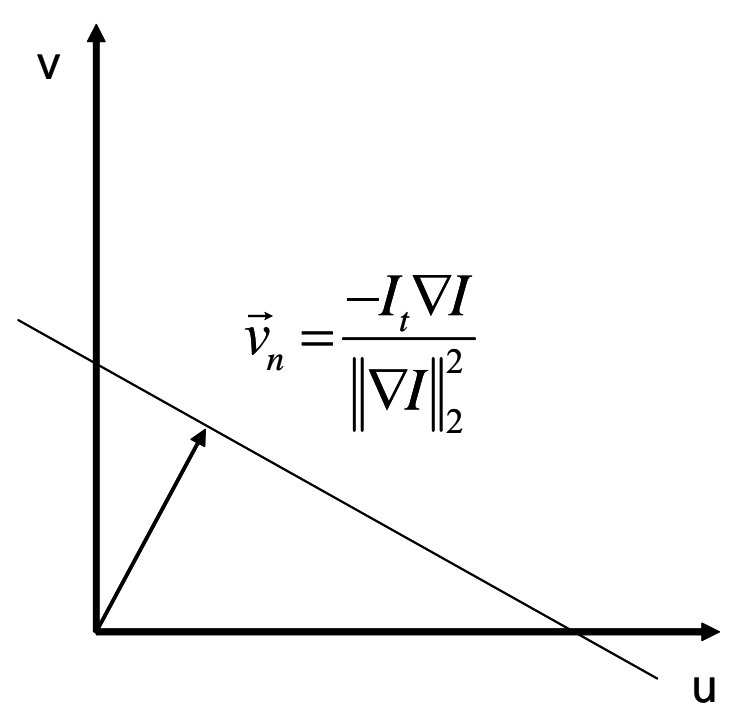

(b)

Figure 4.2: (a) The aperture problem: only normal velocity $\vec{v}_{n}$ can be recovered but tangential velocity $\vec{v}_{t}$ cannot; (b) The motion constraint equation yields a line $\vec{v}=\left(v_{x}, v_{y}\right)^{T}$ space. One of the velocities on this line is the correct one. The velocity with the smallest magnitude is $\vec{v}_{n}$ [55].

The magnitude and direction of the normal velocity $\vec{v}_{n}=v_{n} \hat{n}$ can be computed in terms of the intensity derivatives $I_{x}, I_{y}$ and $I_{t}$ as: 
$v_{n}=\frac{-I_{t}}{\|\nabla I\|^{2}}$ and $\hat{n}=\frac{\left(I_{x}, I_{y}\right)}{\|\nabla I\|^{2}}$

where $v_{n}$ and $\hat{n}$ are the raw normal velocity magnitude and raw normal velocity direction, respectively, i.e.:

$\vec{v}_{n}=v_{n} \hat{n}=\frac{-I_{t}\left(I_{x}, I_{y}\right)}{\|\nabla I\|_{2}^{2}}$

is the raw normal velocity and $\nabla I=\left(I_{x}, I_{y}\right)$ is the spatial intensity gradient.

2D Horn-Schunck: Horn and Schunck [55, 54] combined the gradient constraint equation with a global smoothness term to constrain the estimated velocity field $\vec{v}=\left(v_{x}, v_{y}\right)$, minimizing:

$\int_{D}\left(\nabla I . \vec{v}+I_{t}\right)^{2}+\lambda^{2}\left[\left(\frac{\partial v_{x}}{\partial x}\right)^{2}+\left(\frac{\partial v_{x}}{\partial y}\right)^{2}+\left(\frac{\partial v_{y}}{\partial x}\right)^{2}+\left(\frac{\partial v_{y}}{\partial y}\right)^{2}\right] d x d y$

defined over the image domain, where the magnitude $\lambda$ reflects the relative influence of the smoothness term. Iterative equations are used to minimize above equation and the image velocity can be obtained from the Gauss Seidel equations that solve the appropriate Euler-Lagrange equations:

$$
\begin{aligned}
& v_{x}^{k+1}=\bar{v}_{x}^{k}-\frac{I_{x}\left[I_{x} \bar{v}_{x}^{k}+I_{y} \bar{v}_{y}^{k}+I_{t}\right]}{\alpha^{2}+I_{x}^{2}+I_{y}^{2}} \\
& v_{y}^{k+1}=\bar{v}_{y}^{k}-\frac{I_{y}\left[I_{x} \bar{v}_{x}^{k}+I_{y} \bar{v}_{y}^{k}+I_{t}\right]}{\alpha^{2}+I_{x}^{2}+I_{y}^{2}}
\end{aligned}
$$


where $\mathrm{k}$ denotes the iteration number, $\bar{v}_{x}^{0}$ and $\bar{v}_{y}^{0}$ denote initial velocity estimate which are set to zero and $\bar{v}_{x}^{k}$ and $\bar{v}_{y}^{k}$ denote neighborhood averages of $v_{x}^{k}$ and $v_{y}^{k}$.

Figure 4.3 (a) and (b) show two fingerprint images and 4.3 (c) optical flow determined by Horn-Schunck method. 

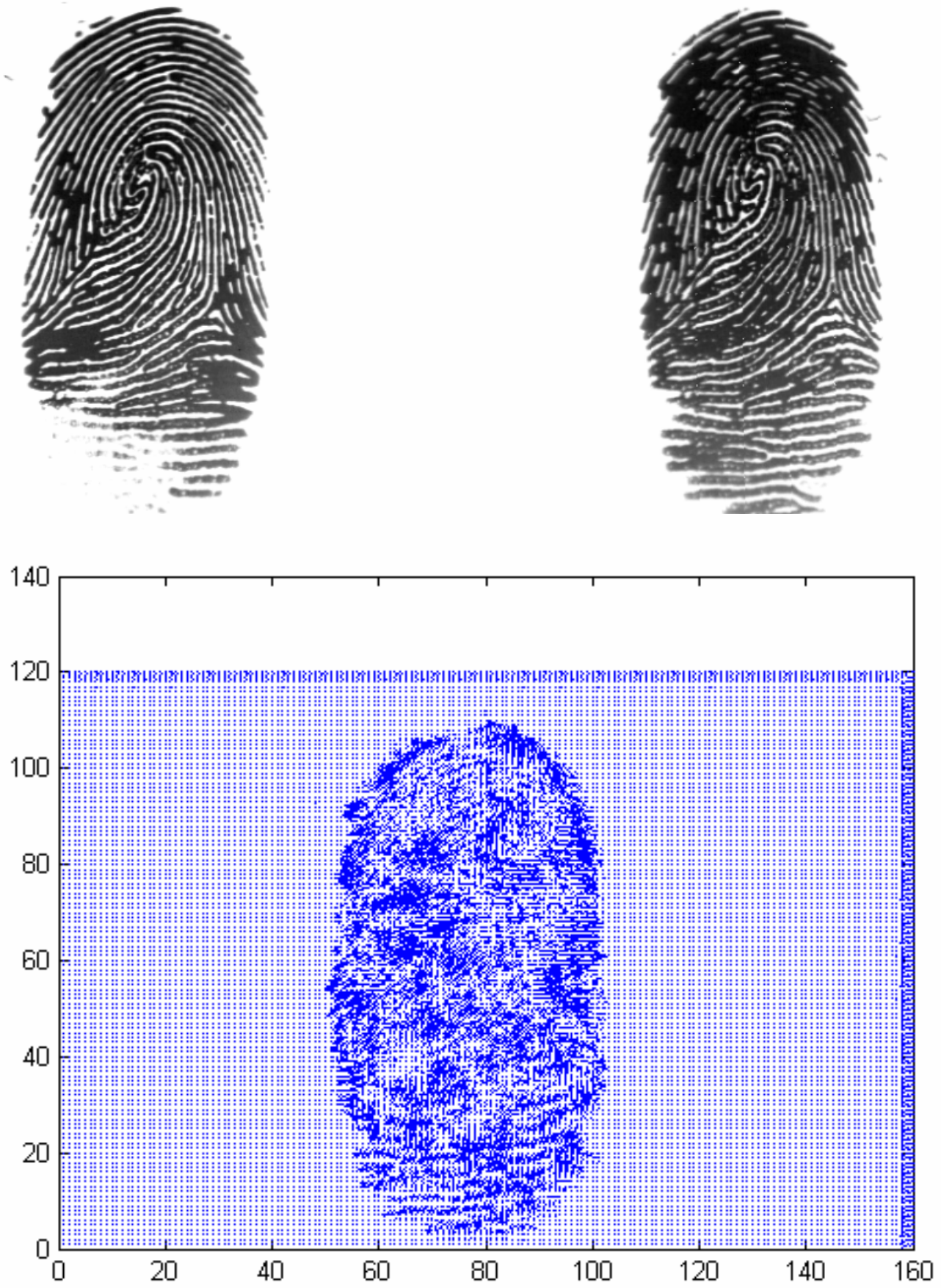

Figure 4.3: (a) and (b) Two fingerprint imq(e) after affine transform, (c) optical flow determined by Horn-Schunck method. 


\subsection{Multi-impression fingerprint enhancement}

Multi-impression fingerprint enhancement can be performed either on binary level images or grey level images. In binary level images, ridge pixels are assigned value 1 and non-ridge pixels are assigned value 0 . Binary level image can be obtained by applying ridge extraction algorithm on the grey level image. Enhancement on binary level image has its inherent limitations because, after applying ridge extraction algorithm on original grey level image, information about true ridge structures is often lost depending on the performance of ridge extraction algorithm.

Binary-level image enhancement: We propose the following steps for the binarylevel fingerprint image enhancement:

1. Align the two fingerprint images initially using affine transformation.

2. After affine transformation align them using Horn-Schunck method for determining optical flow, to account for elastic non-linear deformation.

3. Now, determine the low quality regions in the fingerprint images using coherence of direction as explained in chapter 3 .

4. Convert the grey-level images to binary-level images using a suitable ridge extraction algorithm such that ridge pixels are assigned value 1 and non-ridge pixels are assigned value 0 .

5. As the probability of same local region to be of low quality in both fingerprints images of a same user is very low, so copy the ridge map which is not detected as poor in one image to another image where that region is detected as of poor quality. 
Results are shown in next chapter.

Grey-level image enhancement: Initial steps of alignment in case of grey-level fingerprint image enhancement are same as in case of binary-level fingerprint image enhancement. Following are the steps we propose in case of grey-level fingerprint image enhancement:

1. Align the two fingerprint images initially using affine transformation.

2. After affine transformation align them using Horn-Schunck method for determining optical flow, to account for elastic non-linear deformation.

3. Now, determine the low quality regions in the fingerprint images using coherence of direction as explained in chapter 3 and mask the low quality regions.

4. Interpolate the low quality areas using "Sequential Error-Concealment Techniques Using Orientation Adaptive Interpolation", [ref] as explained in previous chapter, but in case of multiple fingerprint images bad pixels are not interpolated from their vicinity, instead from the good quality regions which are located at the same position in the aligned fingerprint image, as the vicinity of masked low quality regions in the fingerprint image to be enhanced.

Results are shown in next chapter. 


\section{Chapter 5: Results}

We have proposed Multi-impression enhancement of fingerprint images in this thesis. Its whole motive is to enhance the fingerprint images i.e., to improve the local low quality regions in a fingerprint image such as (wet areas, dry areas, etc.). We believe that results can be more appreciated by the visual comparison of original images and the enhanced images.

Before showing the results of multi-impression enhancement, let us appreciate the results for the individual modules which lead us to the multi-impression enhancement. As explained in chapter 3, first step is to detect the location of low quality regions. In chapter 4 we proposed single impression enhancement using the Orientation Adaptive Interpolation which basically interpolates the low quality regions from their vicinity. Finally, multi-impression enhancement, before which we need to do the image alignment and then interpolation of low quality regions from the neighborhood in other aligned fingerprint image.

\subsection{Fingerprint Quality Assessment}

To detect the location of local low quality regions present in a fingerprint image we proposed to use block-wise coherence of direction. Our method is based on coherence of direction, as in the local regions which are either wet, dry or have scars coherence will be considerably lower as compared to the regions of good quality where ridge and valleys are clearly visible. 


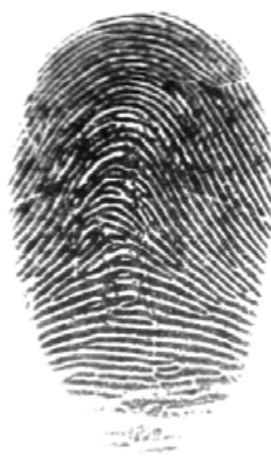

(a)

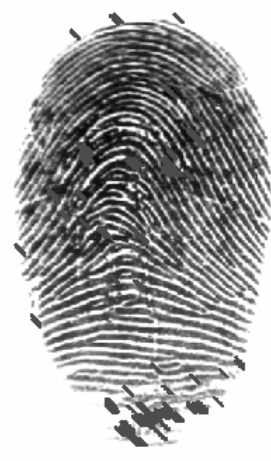

(c)

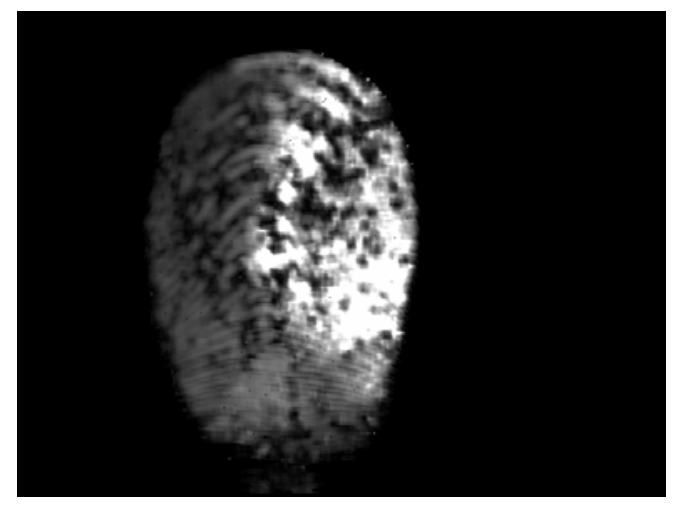

(b)

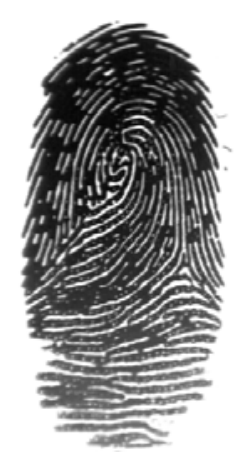

(d)

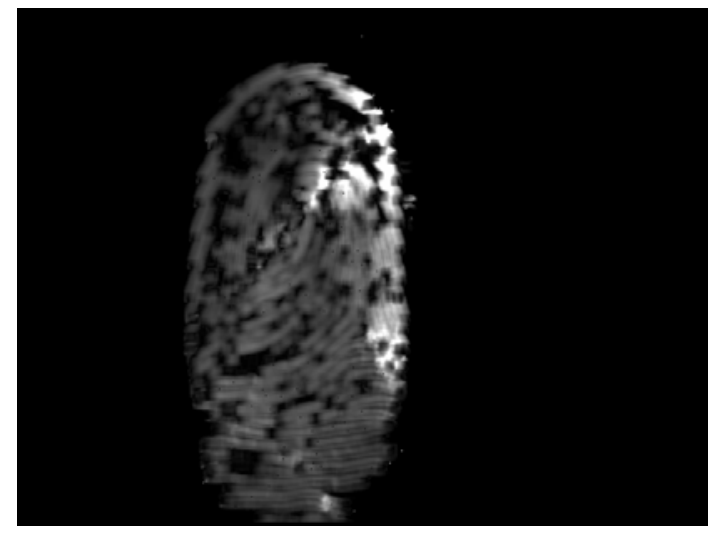

(e)

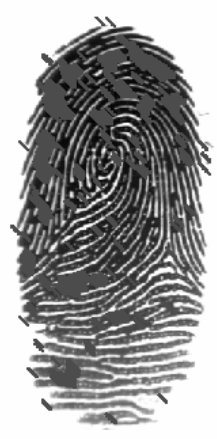

(f) 


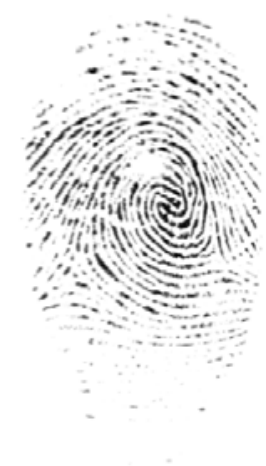

(g)

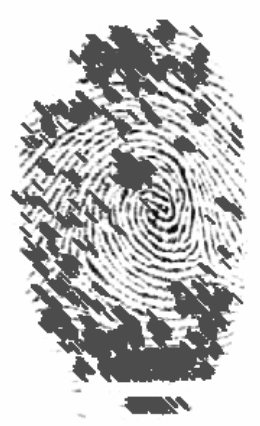

(i)

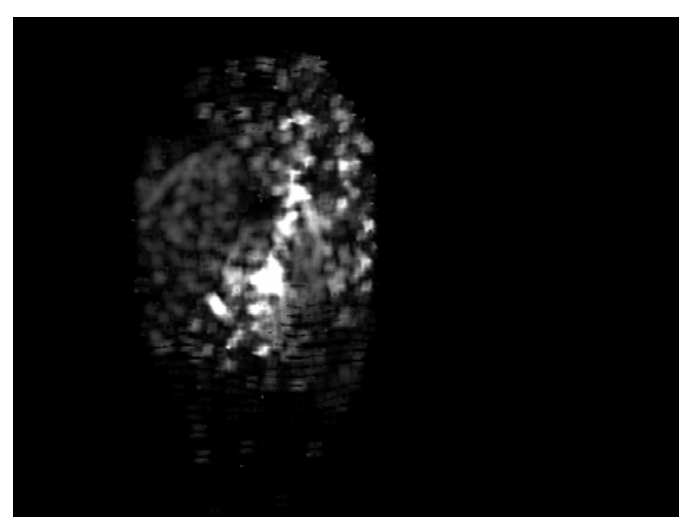

(h)

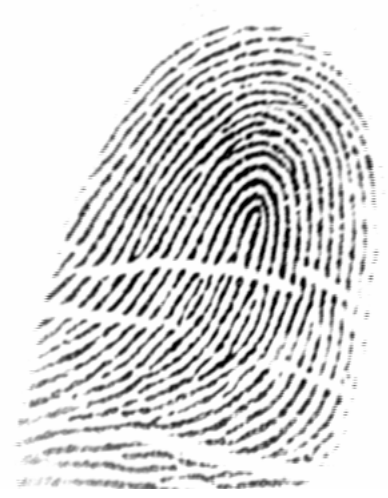

(j)

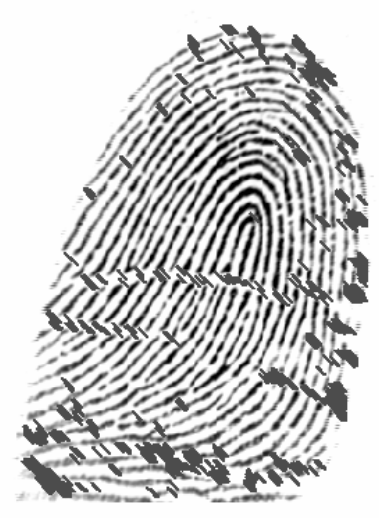

(l)

Figure 5.1 (a), (d), (g), (j) are fingerprints of good quality, with wet areas, with dry areas and scars respectively; (b), (e), (h), (k) show their coherence map respectively and; (c), (f), (i), (l) show the detected low quality regions 


\subsection{Single Impression Fingerprint Enhancement}

After low quality regions are detected, first of all we propose to do single impression enhancement. In single impression enhancement we propose to interpolate low quality regions from their vicinity using "Orientation Adaptive Interpolation", which was explained in detail in chapter 3 . When local low quality regions are detected, a binary mask is generated which mask the low quality regions to be enhanced. These masked low quality regions are then interpolated from their vicinity. 
1. Fingerprint image with wet areas

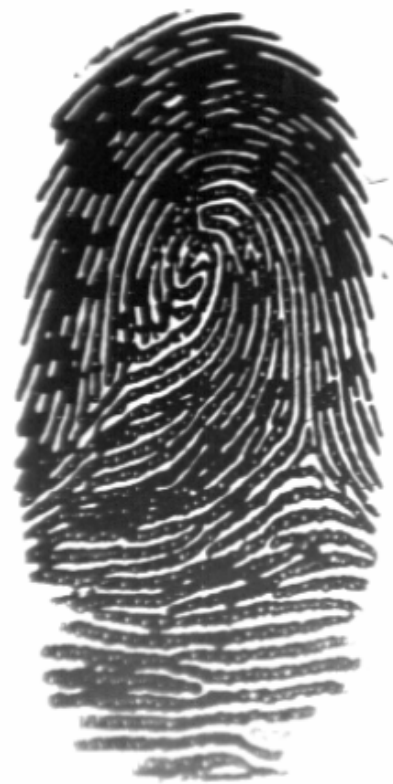

(a)

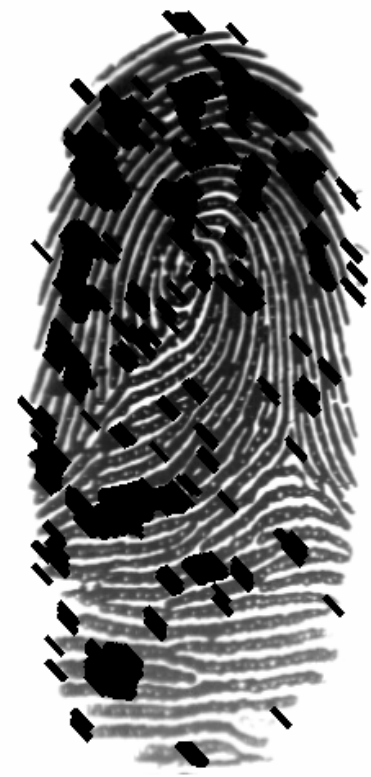

(c)

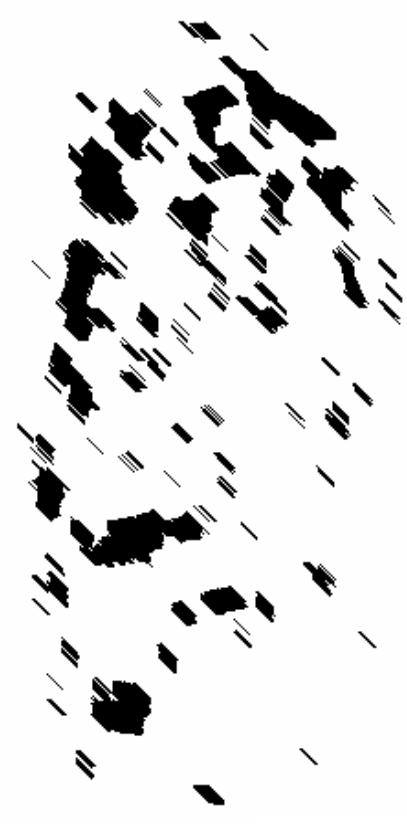

(b)

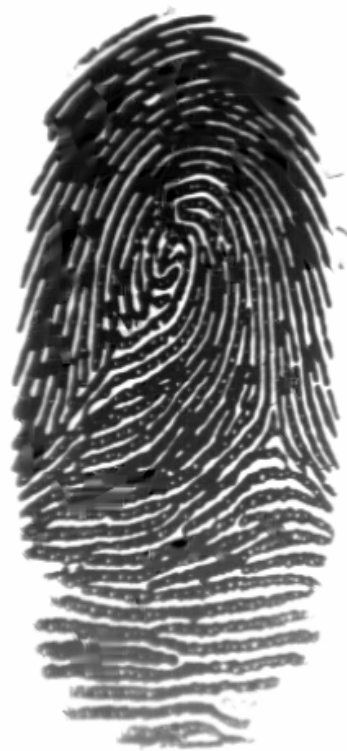

(d)

Figure 5.2: (a) Original fingerprint image with wet areas; (b) Binary mask for low quality regions; (c) Masked low quality regions; (d) Image after interpolation of masked areas. 
2. Fingerprint image with dry areas

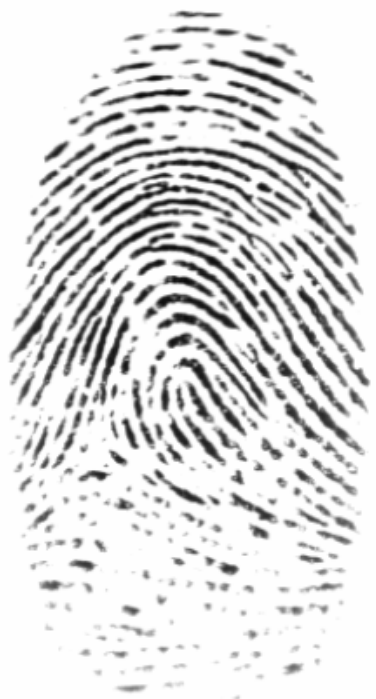

(a)

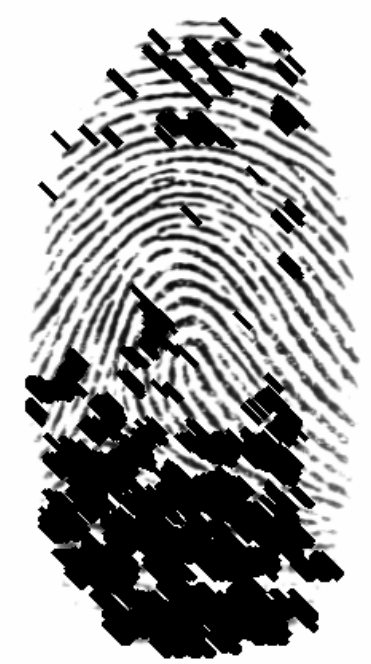

(c)

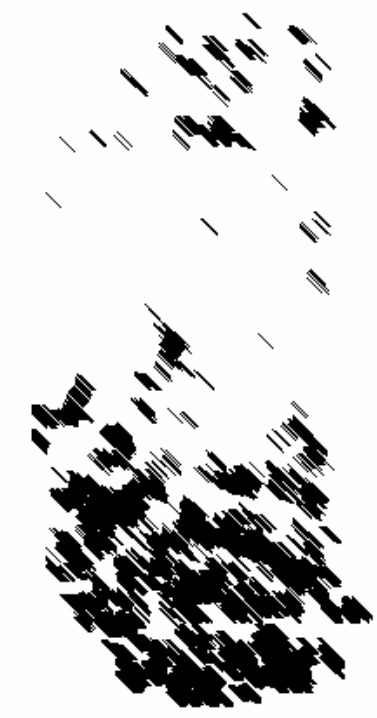

(b)

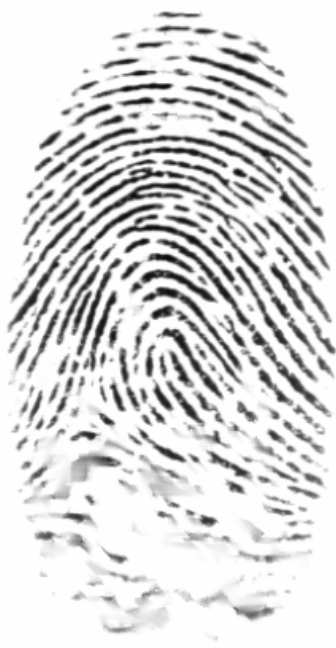

(d)

Figure 5.3: (a) Original fingerprint image with dry areas; (b) Binary mask for low quality regions; (c) Masked low quality regions; (d) Image after interpolation of masked areas. 
3. Fingerprint image with scars

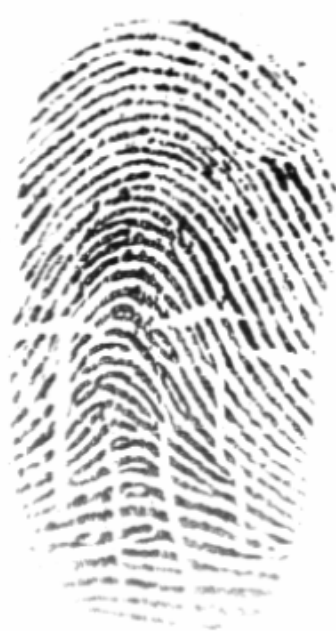

(a)

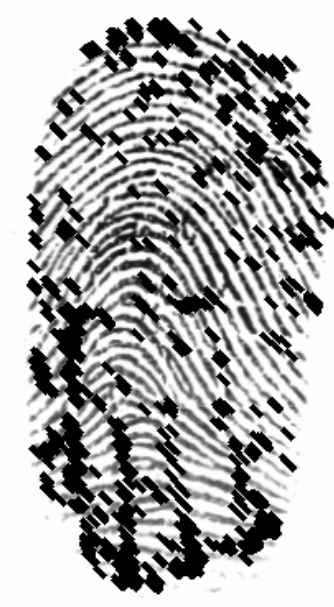

(c)

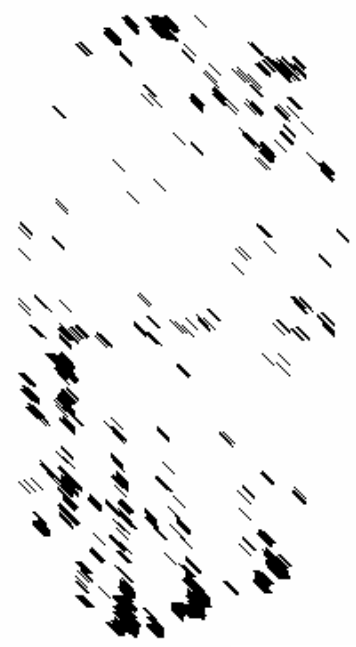

(b)

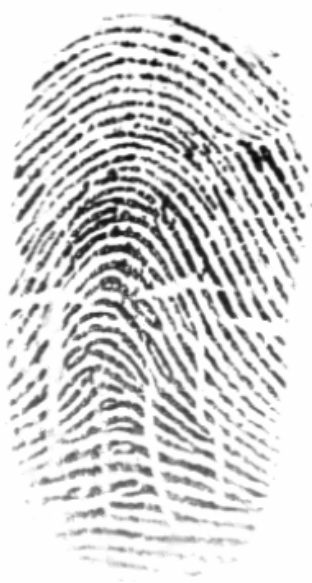

(d)

Figure 5.4: (a) Original fingerprint image with scars; (b) Binary mask for low quality regions; (c) Masked low quality regions; (d) Image after interpolation of masked areas. 
Visually we can see that there is not much difference between the original image and the enhanced fingerprint image, this may be due to presence of so many consecutive lost blocks present in the region to be repaired. Hence, we came up with the technique of multi-impression enhancement of fingerprint images.

\subsection{Multi-Impression Fingerprint Enhancement}

In this technique, a reference image and an image to be enhanced of same class are taken. Before the enhancement process, first of all it is necessary to align the two images as close as possible. After alignment is done, there can be binary-level fingerprint image enhancement and grey-level fingerprint enhancement.

\subsubsection{Image Alignment}

- Optical Flow based Alignment: After applying affine transformation and scaling, we proposed to use Horn-Schunck method for determining optical flow for further alignment, as explained in chapter 4. Optical flow based alignment is a non-parametric technique i.e., it does not based on image features. It is an iterative technique. Basically, it is based on regularized minimization of the distance between two images. Figure 5.5 shows the alignment results for fingerprint image with wet areas, after affine transform and scaling and after applying Horn-Schunck method. 
1. Fingerprint image with wet areas

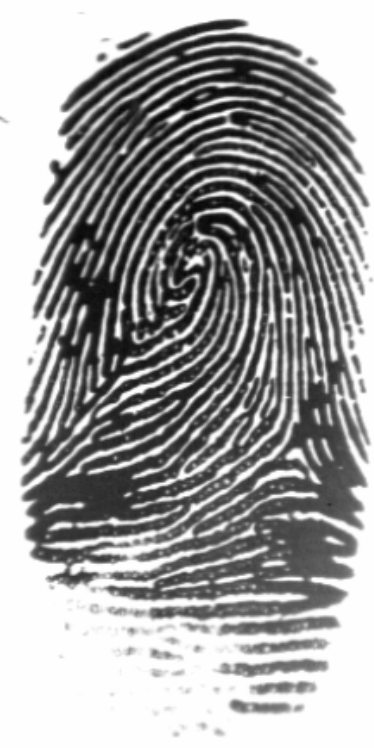

(a) Reference Image

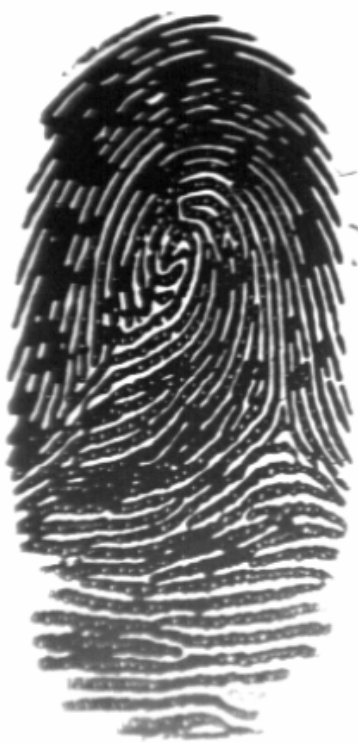

(b) Image to be enhanced

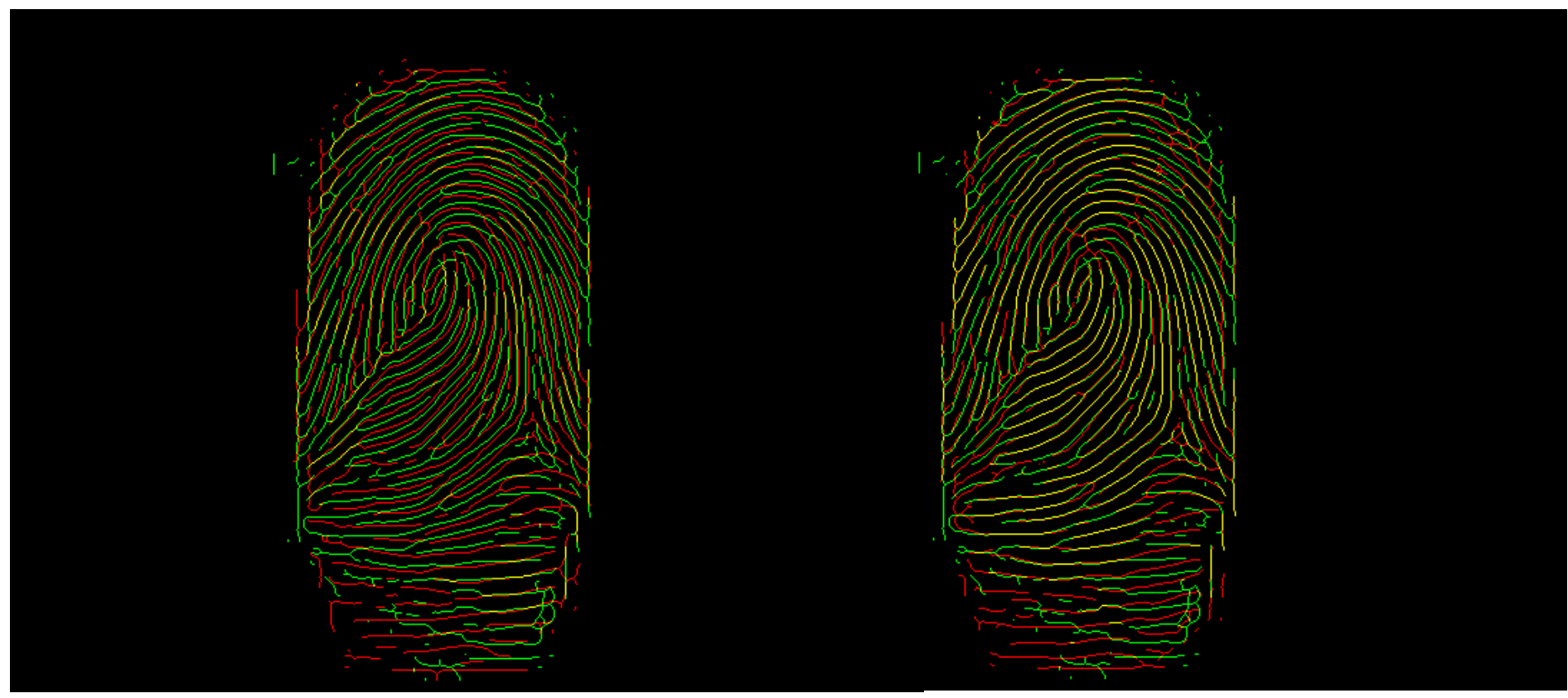

(c) Alignment after affine transformation and scaling (d) Alignment after applying Horn-Schunck method

Figure 5.5 Fingerprint image alignment with wet areas. 
- Thin Plate Spilne based Alignment: Figure 5.6 shows the alignment of two fingerprint images with wet areas, using Thin Plate Splines (TPS). This is a landmark based technique based on the locations of minutiae points and the correspondence between them. Alignment using TPS is non-iterative but on the other hand if the point correspondence is incorrect it can result in wrong warping of the image.

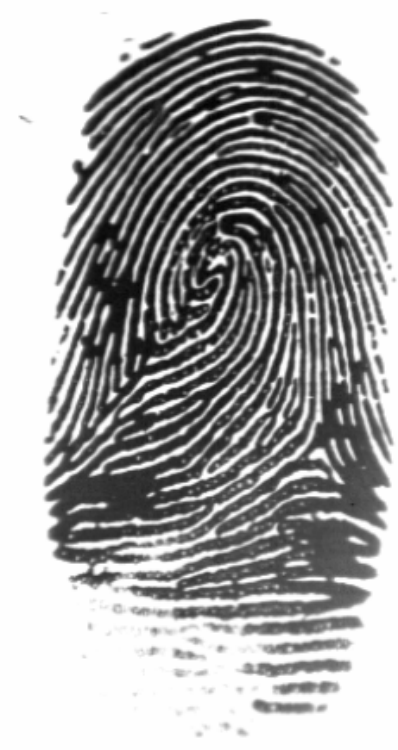

(a) Reference Image

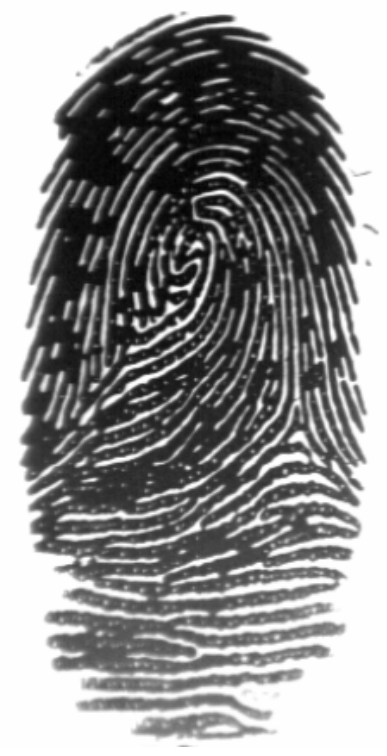

(b) Image to be enhanced 


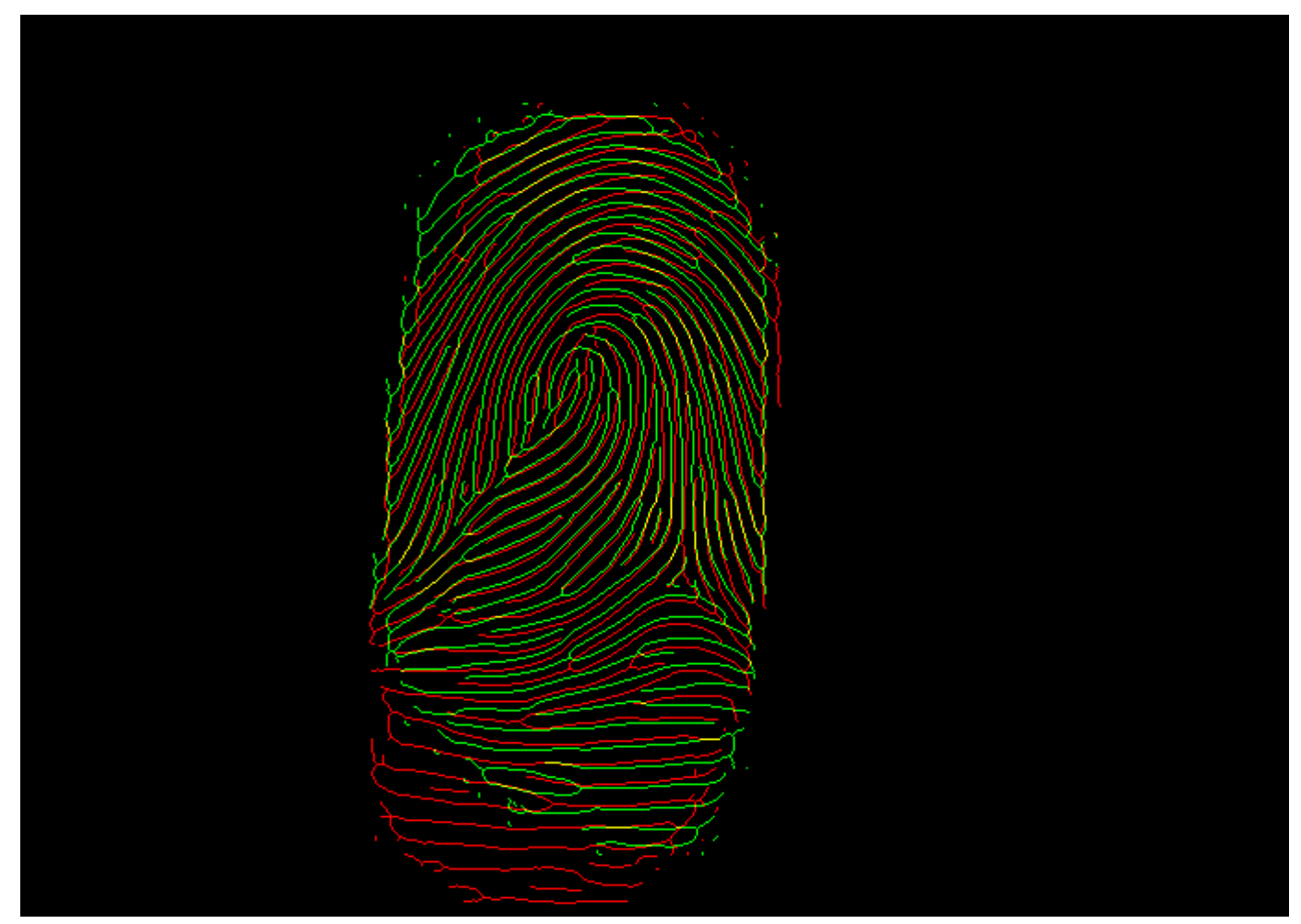

(c) Alignment after using Thin Plate Splines (TPS)

Figure 5.6: Thin Plate Splines (TPS) based fingerprint image alignment.

Figure 5.7 shows the alignment results for fingerprint image with dry areas, after affine transform and scaling and after applying Horn-Schunck method. Thin Plate Splines (TPS) based alignment technique was not able to align these pair of images with dry areas, which may be due to wrong minutiae correspondence information. 
2. Fingerprint image with dry areas.

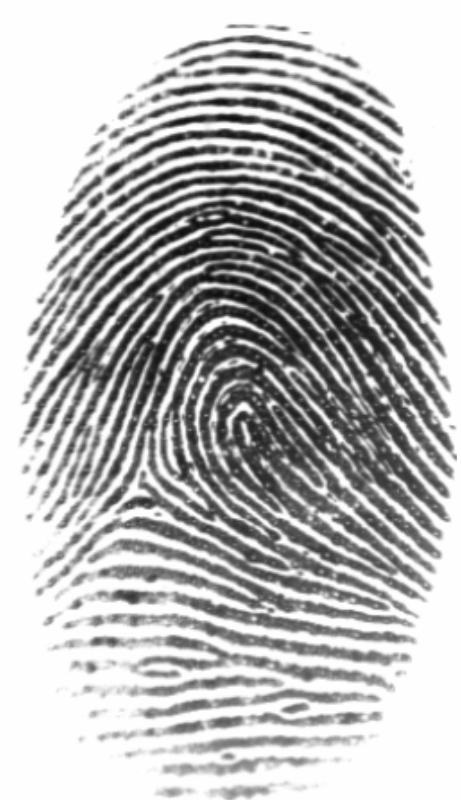

(a) Reference Image

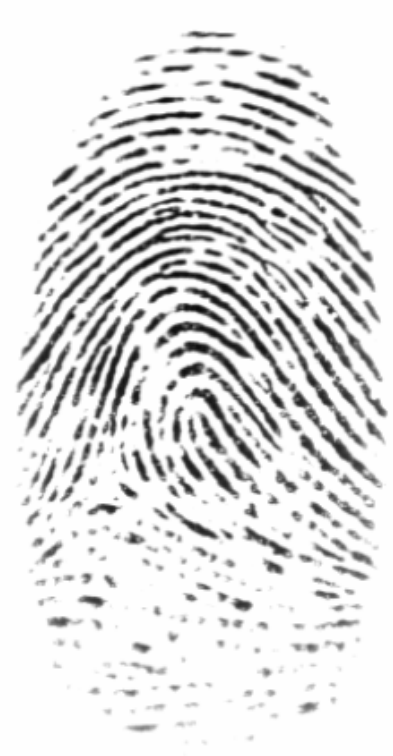

(b) Image to be enhanced

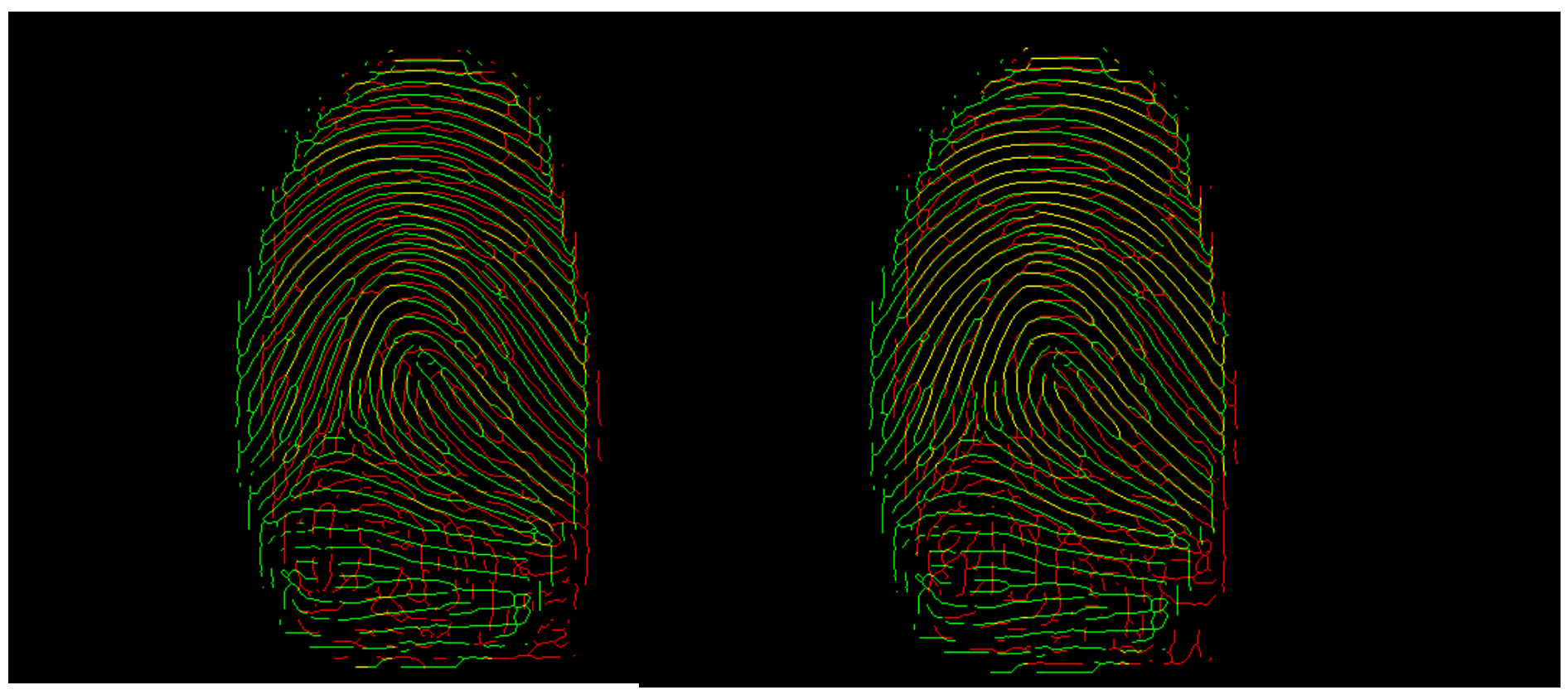

(c) Alignment after affine transformation and scaling (d) Alignment after applying Horn-Schunck method

Figure 5.7 Fingerprint image alignment with dry areas. 


\subsubsection{Binary-level fingerprint image enhancement}

In the following sub section we present the results of binary-level fingerprint enhancement, after following the steps as explained in chapter 4, section 4.2.

1. Fingerprint image with wet areas

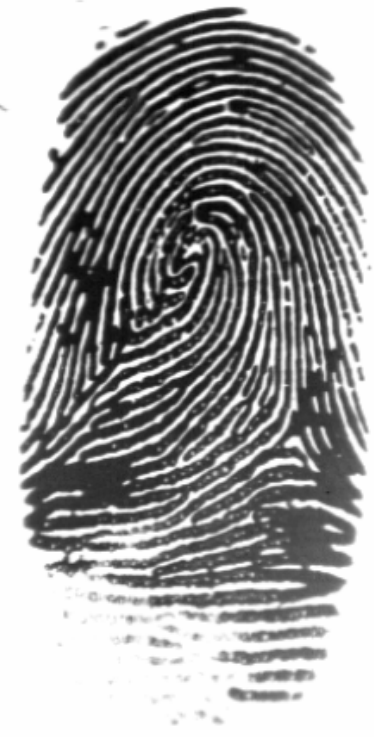

(a) Reference Image

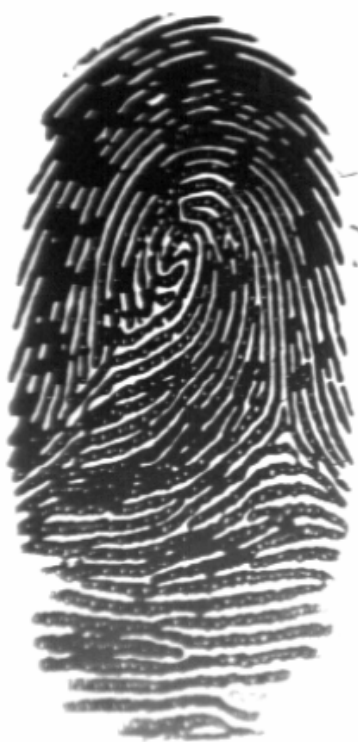

(b) Image to be enhanced

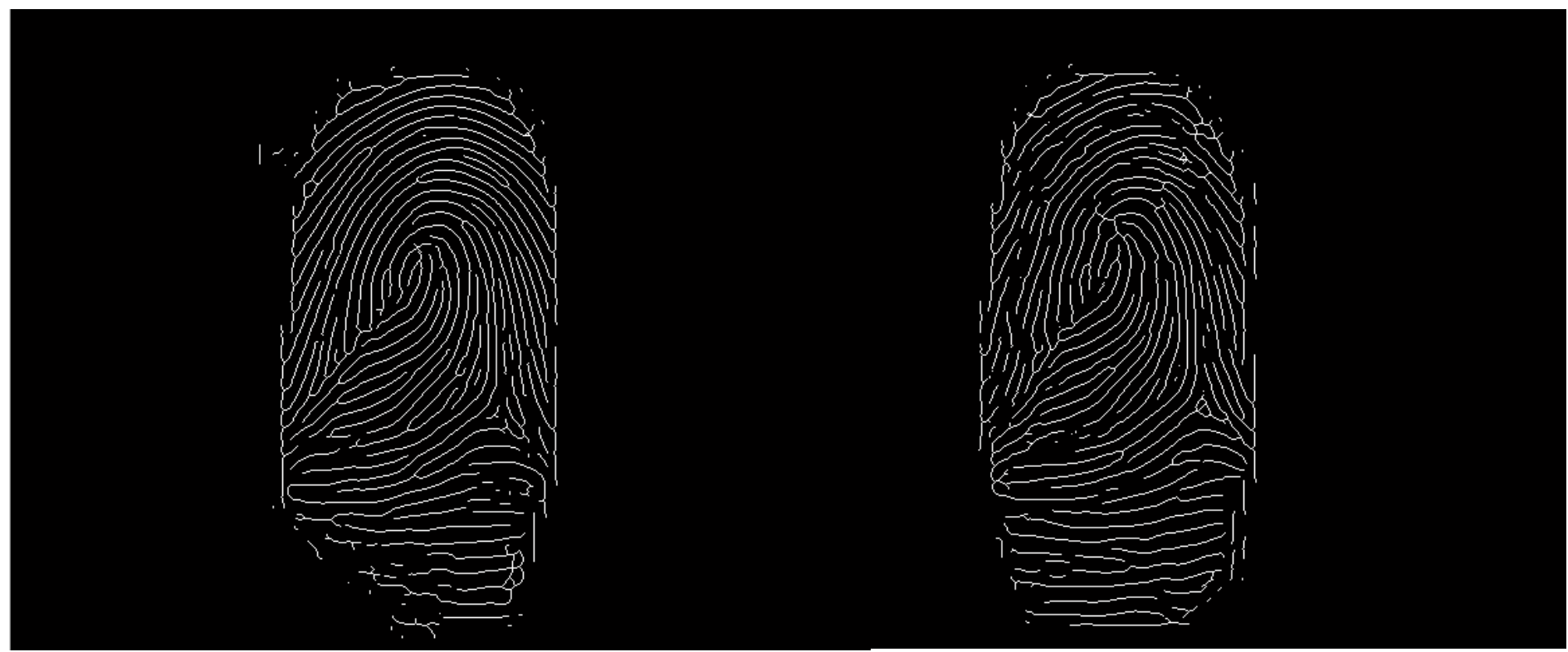

(c) Thinned Binary-level image of reference image (d) Thinned Binary-level image of image with wet areas before enhancement 


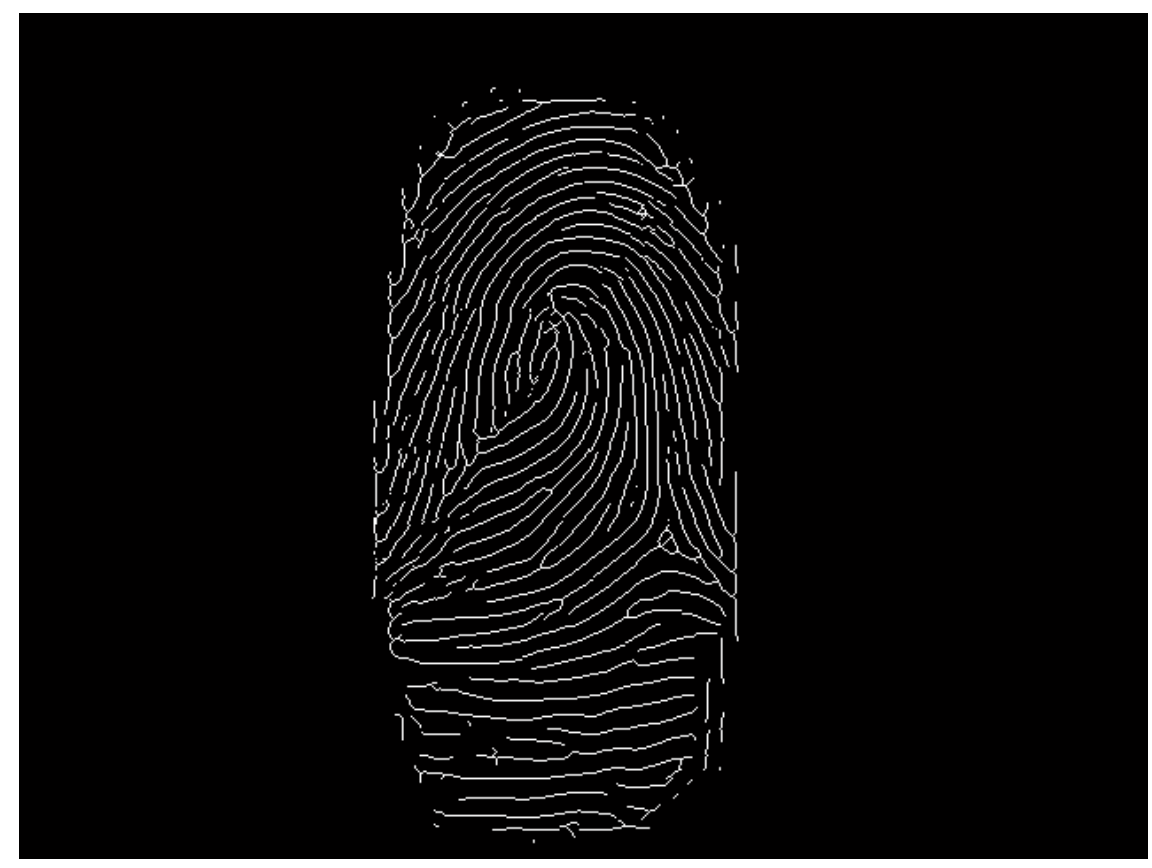

(e) Thinned Binary-level image of image with wet areas after enhancement

Figure 5.8 Binary level enhancement of fingerprint image with wet areas

3. Fingerprint image with dry areas.

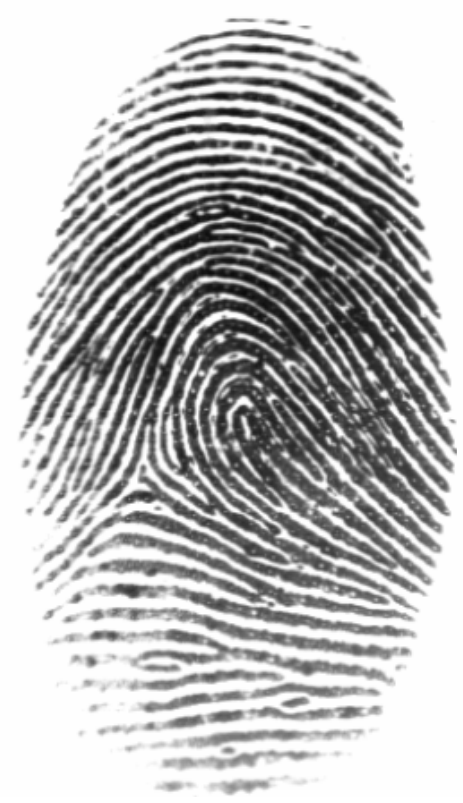

(a) Reference Image

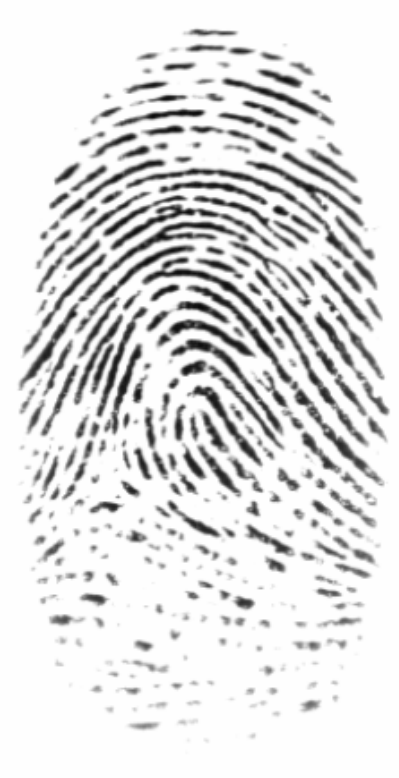

(b) Image to be enhanced 

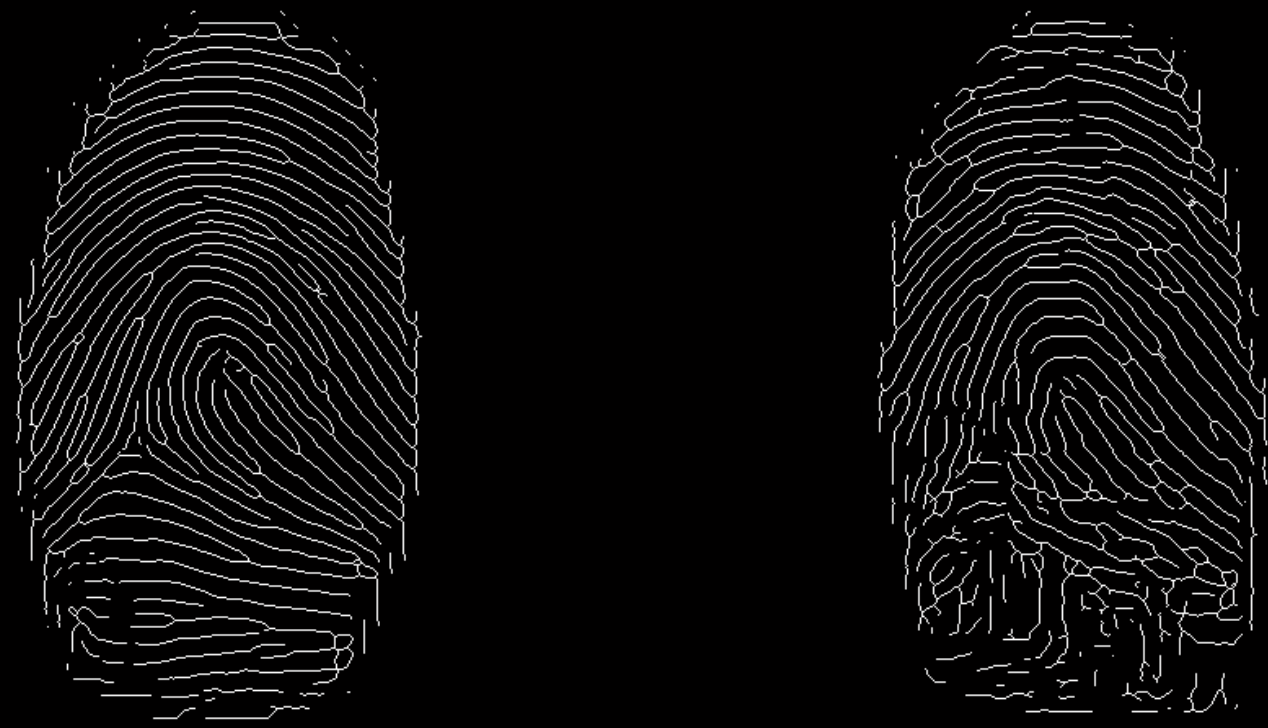

(c) Thinned Binary-level image of reference image

(d) Thinned Binary-level image of image with dry areas before enhancement

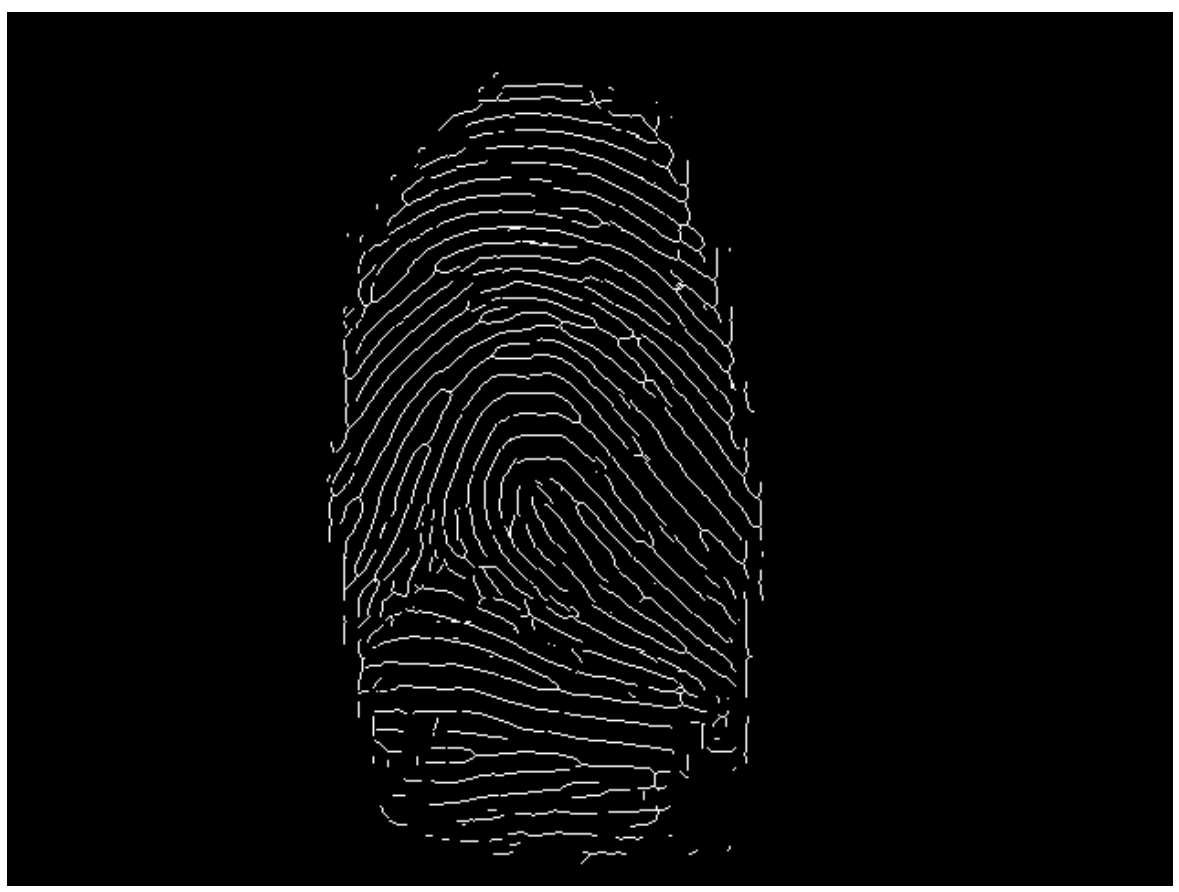

(e) Thinned Binary-level image of image with dry areas after enhancement

Figure 5.9 Binary level enhancement of fingerprint image with dry areas 


\subsubsection{Grey-level fingerprint image enhancement}

In the following sub section we present the results of binary-level fingerprint enhancement, after following the steps as explained in chapter 4, section 4.2.

1. Fingerprint image with wet areas:

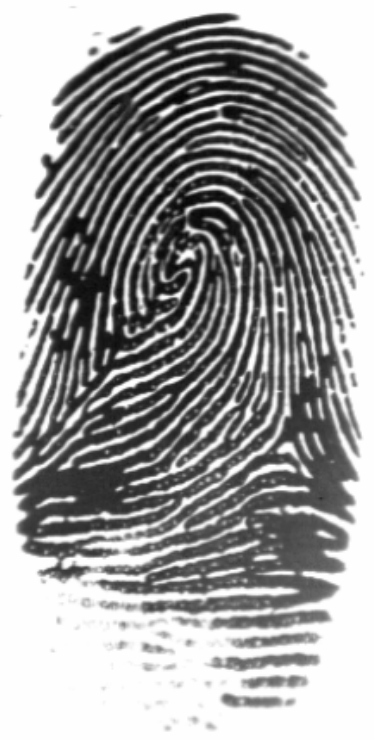

(a) Reference Image

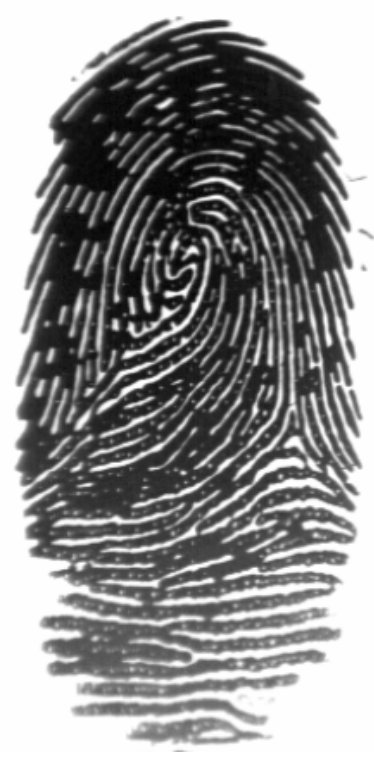

(b) Image to be enhanced 


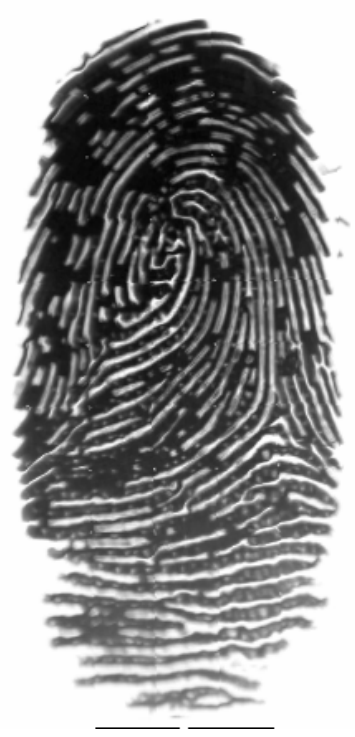

(c) Image after alignment

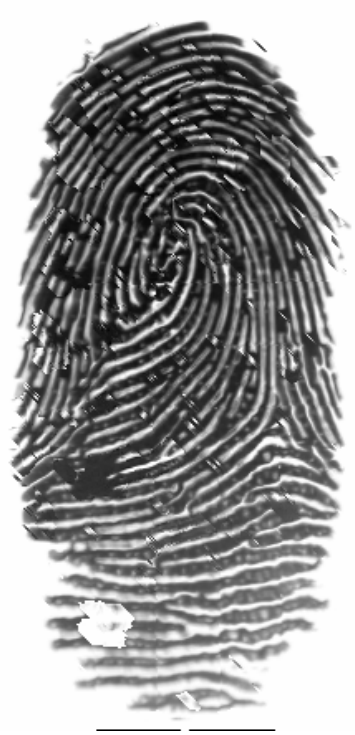

(d) Enhanced image

Figure 5.10 Grey level enhancement of fingerprint image with wet areas.

2. Fingerprint image with dry areas:

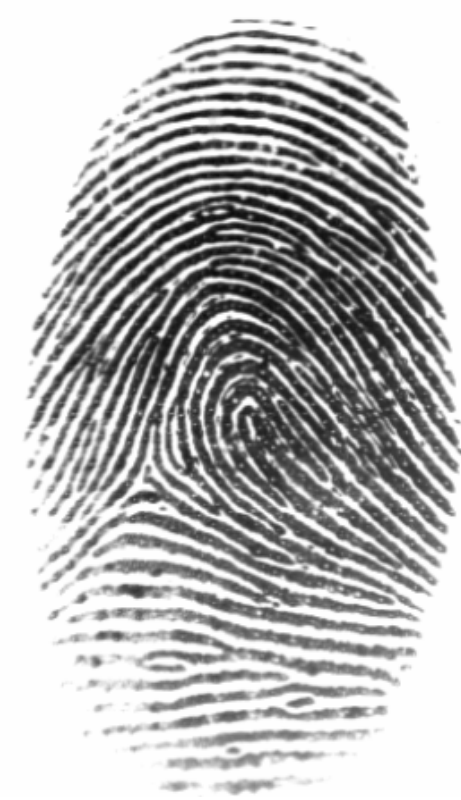

(a) Reference Image

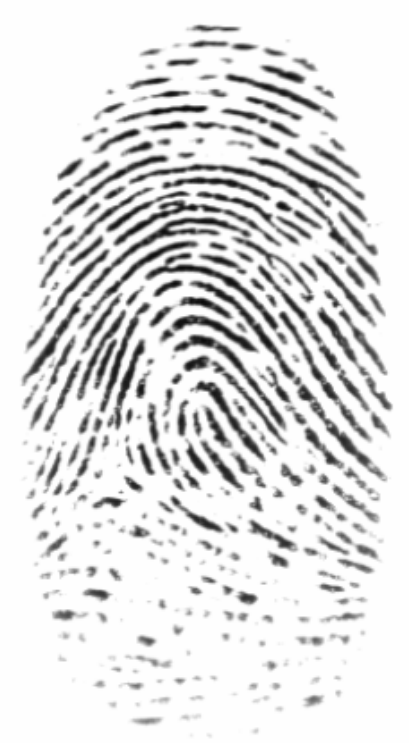

(b) Image to be enhanced 

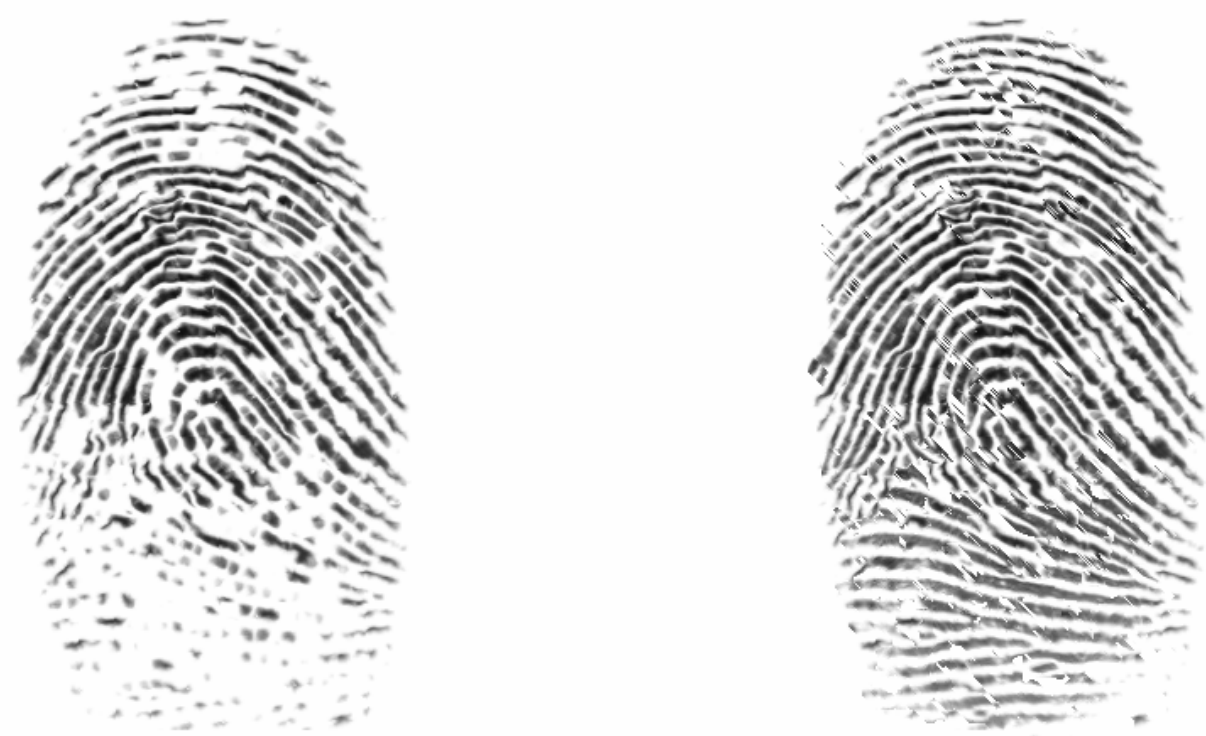

\section{(c) Image after alignment}

(d) Enhanced image

Figure 5.11 Grey level enhancement of fingerprint image with dry areas.

We can see that there are some artifacts introduced during alignment process which continue to follow further in case of fingerprint image with dry areas. This might be due the reason Horn-Schunck method does not work well in this case as the intensity difference is very significant between reference image and the image to be enhanced. Reference image is very dark as compared to the image to be enhanced. A better alignment technique should not introduce such artifacts.

We did not perform multi-impression enhancement on the fingerprint images with scars, as our intuition that probability of same local regions to be present at same location in multiple fingerprint images is very low, does not holds true in this case. Location of scars with respect to the finger does not change; hence this technique cannot be used in case of fingerprint image with scars. 


\subsection{Performance Evaluation}

To validate our multi-impression enhancement technique we have tried to incorporate it into Filterbank-Based Fingerprint Matching [58]. This algorithm uses a bank of Gabor filters to capture both local and global details in a fingerprint image as a compact fixed length fingercode. The fingerprint matching is based on the Euclidean distance between the two corresponding Fingercodes.

Fingerprint images of 10 subjects with 8 images per subject were used from FVC 2004 DB1 database. Images of each user were enhanced by applying proposed multiimpression enhancement technique. Figure 5.12 shows the ROC curve plotting False Accept Rate (FAR) and False Reject Rate (FRR). An overall improvement of $1.85 \%$ in Equal Error Rate (EER) is observed.

\begin{tabular}{|l|c|c|}
\hline \multicolumn{1}{|c|}{$\begin{array}{c}\text { Performance } \\
\text { Evaluation }\end{array}$} & Original Fingerprint & $\begin{array}{c}\text { Enhanced Fingerprint } \\
\text { Images }\end{array}$ \\
\hline Equal Error Rate (EER) & $\mathbf{9 . 6 \%}$ & $\mathbf{7 . 7 4 \%}$ \\
\hline d-prime & $\mathbf{1 . 3 8 6 7}$ & $\mathbf{1 . 5 1 4 5}$ \\
\hline
\end{tabular}

Table 5.1: EER and d-prime values before and after enhancement. 


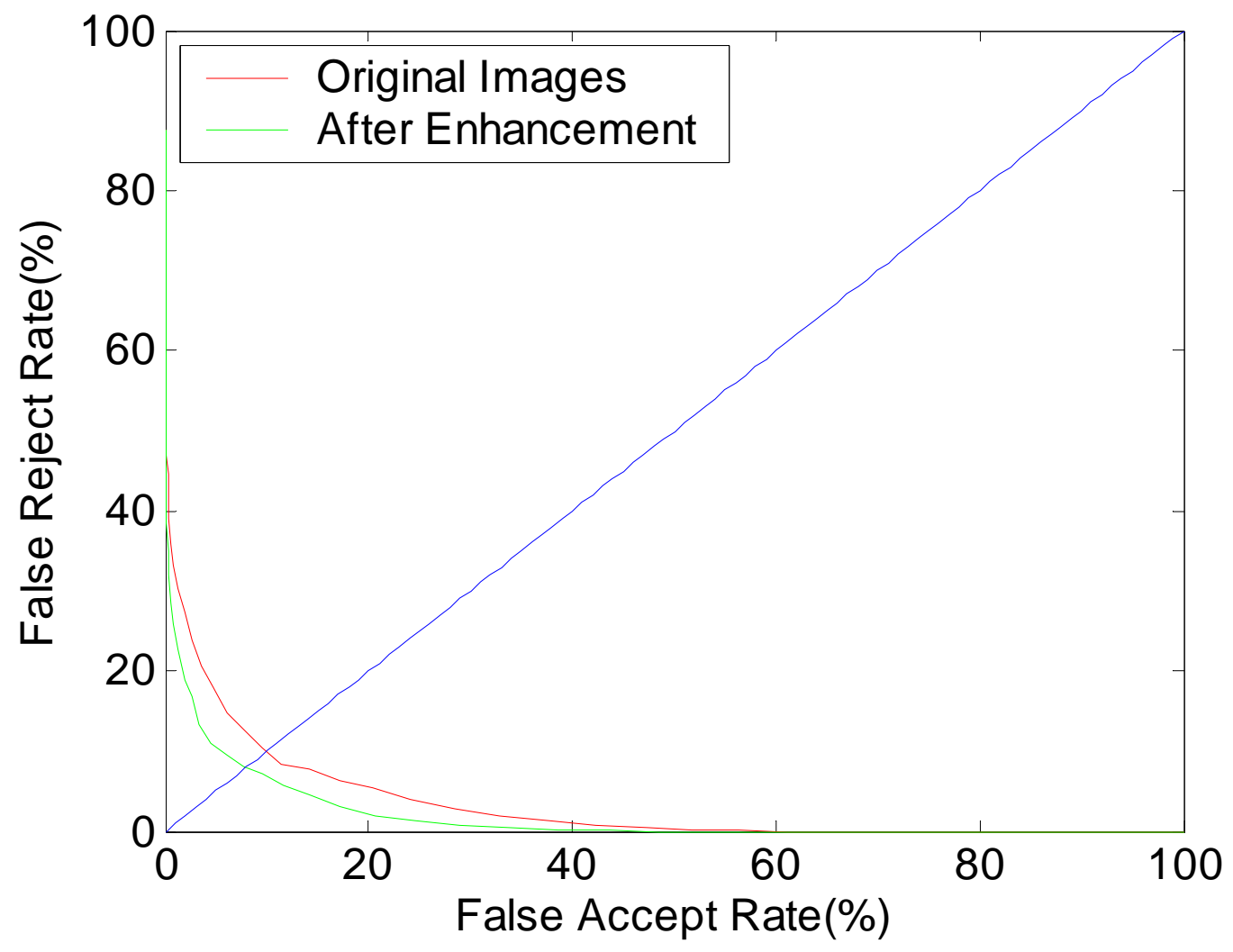

Figure 5.12: ROC curve plotting FAR and FRR 


\section{Chapter 6: Conclusion and Future Work}

\subsection{Conclusion}

With the advancement of Biometrics, due to increase in frauds and crimes, more and more biometric modalities are emerging. Fingerprints are widely used in many personal identification systems due to its permanence and uniqueness. Automatic fingerprint identification systems are being commercially deployed at many places. Hence, error rate of just $1 \%$ may prove to very disastrous. Currently, many researchers are trying to develop systems with $100 \%$ recognition rate.

One of the very important factors which can affect the performance of a fingerprint identification system is the quality of the input images. Several factors determine the quality of fingerprint image: skin conditions (e.g. wetness, dryness, dirtiness, temporary or permanent cuts or bruises), sensor conditions (e.g. dirtiness, noise, size), user cooperation etc. Poor image quality leads to many pseudo-minutiae. This degrades the performance of fingerprint recognition system. In a typical fingerprint identification system first of all acquisition takes place which means acquiring of fingerprint data from the user, then comes the feature extraction part which involves use of various image processing applications and finally matching is done with template feature sets in the database

We propose an economical way to enhance the fingerprint images after the acquisition module i.e., before the feature extraction module. The whole process of enhancement can 
be divided into main parts. First part is the fingerprint quality assessment part, where we detect the location of location of low quality regions in a fingerprint image. Coherence of direction is used for detection as it is considerable low in low quality regions. Coherence of direction method has the advantage of speed and simplicity.

Second part is he fingerprint image enhancement part, i.e., to estimate the ridge flow in the detected low quality regions. It is further subdivided into two kinds of enhancement processes. First kind of enhancement technique is the single-impression fingerprint image enhancement. In this technique we use sequential recovery framework, using orientation adaptive interpolation, to interpolate the detected low quality regions from their vicinity. Sequential recovery has an advantage of preserving important image features such as edges. Since in a detected low quality region in a fingerprint image there are more than one edges and recovery of a pixel not only depends on its available neighbors but also previously recovered pixels, we do not get promising enhancement results in this case of single-impression fingerprint image enhancement.

To overcome the previous problem, we propose the second kind of enhancement technique which is multi-impression fingerprint image enhancement. It is based on the intuition that the probability of same local region to be of low quality in multiple fingerprints images of a same user is very low. But before enhancement, we need to align multiple fames of fingerprint images as close as possible which is very tough due to presence of non-linear elastic deformation. After initially aligning images using affine 
transformation, we propose to use Horn-Schunck method for determining optical flow to further align the fingerprint images.

Now the enhancement can be done of binary-level as well as of grey-level image. In case of binary-level enhancement, we simply copy the binary ridge map of the regions which are not detected as of low quality in one image to another image where that region is detected as of low quality. Visually, new binary ridge map seems to have improved ridge map in the low quality regions. Binary level enhancement has an advantage of speed and simplicity.

In case of grey-level enhancement we use the same orientation adaptive interpolation used in the case of single-impression enhancement. Instead of interpolating pixels from their vicinity we propose to interpolate them from the good quality regions in the other aligned image present at he same location. In this case multiple edges and their orientation in a low quality region are preserved.

Thus the low quality region detection technique gives us the location of low quality regions such as wet areas, dry areas, scars etc. Applying multi-impression fingerprint image enhancement technique helps in estimating ridge flow in the detected low quality region preserving their orientation. 


\subsection{Future Work}

Biometrics is growing at a very fast rate and researchers all over the world are trying to achieve the goal of $100 \%$ recognition rate. A biometric system consists of different modules and if improving any module can help in achieving the goal, it will be a stepping stone to future works.

We have tried to assess the quality of a fingerprint image which may prove to be very useful during image registration. We have come up with the enhancement of local low quality regions of a fingerprint image using multiple impressions. We have merged different methodologies to come with this enhancement technique. Still there are areas that need to be explored further.

We have used multiple impressions to enhance local low quality regions of a fingerprint image. We have aligned two fingerprint images before the enhancement could be done. For alignment we estimate affine parameters rotation and translation and scaling to align the fingerprint images. Then we have used Horn-Schunck method for determining optical flow to provide further robust alignment. One issue not addressed is that fingerprints are an elastic material, i.e., there is bound to be some elastic non-linear deformation during image acquisition process. We believe that if this non-linear deformation is considered it can provide us more robust alignment and can improve the enhancement further.

Visually we can see that in enhanced local low quality areas ridge flow and orientation is preserved but another important work that can be done further is to incorporate this 
enhancement technique in a fingerprint identification system and how much it can improve the performance of the system.

These are the issued that have to be addressed to later date. There may be other avenues which might need to be explored further and I would thank readers to make me aware of them. 


\section{References:}

[1] A. K. Jain, A. Ross and S. Prabhakar, "An Introduction to Biometric Recognition", IEEE Transactions on Circuits and Systems for Video Technology, Special Issue on Image- and Video-Based Biometrics, Vol. 14, No. 1, pp. 4-20, January 2004.

[2] "Biomtric FAQ," [Online] Available at:

www.bromba.com/faq/biofaqe.htm, as on: 02/13/2006

[3] D. Maltoni, D. Maio, A. K. Jain, S. Prabhakar, Handbook of Fingerprint Recognition, Springer 2003

[4] N. Wu; J. Zhou, "Model based algorithm for singular point detection from fingerprint images", Image Processing, 2004. ICIP '04. 2004 International Conference on Volume 2, 24-27 Oct. 2004 Page(s):885 - 888

[5] F. Alonso-Fernandez, J. Fierrez-Aguilar, and J. Ortega-Garcia, "A review of schemes for fingerprint image quality computation", Proc.3rd COST 275 Workshop, Biometrics on the Internet, European Commission, 2005.

[6] L. Hong, Y. Wan and A. K. Jain, "Fingerprint image enhancement: Algorithm and performance evaluation", IEEE Trans. on PAMI, 20(8):777-789, August 1998.

[7] E. Lim, X. Jiang; and W. Yau "Fingerprint quality and validity analysis", IEEE Proc. ICIP, 1:469-472, September 2002.

[8] Y. Chen, S. Dass, and A. K. Jain, "Fingerprint quality indices for predicting authentication performance", Proc. AVBPA, pp. 160-170, Rye Brook, NY, July 2005.

[9] L. Shen, A. C. Kot, and W. M. Koo, "Quality measures of fingerprint images", Proc. AVBPA: 266-271, 2001.

[10] N. Ratha and R. Bolle (Eds.). Automatic Fingerprint Recognition Systems. Springer-Verlag, N.York, 2004.

[11] A. Ross, S. Dass and A. K. Jain, "A Deformable Model for Fingerprint Matching”, Pattern Recognition, Vol. 38, No. 1, pp. 95-103, Jan 2005.

[12] A. K. Jain, S. Prabhakar, A. Ross, "Fingerprint Matching: Data Acquisition and Performance Evaluation", MSU Technical Report, No. MSU-CPS-99-14, March 1999. 
[13] L. Hong, A. K. Jain, S. Pankanti, and R. Bolle, "Fingerprint Enhancement", 3rd IEEE Workshop on Applications of Computer Vision (WACV '96) p. 202.

[14] A. Almansa and T. Lindberg, "Fingerprint Enhancement by Shape Space Adaptation of Scale-Space Operators with Automatic Scale Selection", IEEE Transactions on Image Processing, vol. 9, no. 12, December 2000

[15] X. Meihua and W. Zhengming, "Fingerprint Enhancement based on Edge Directed Diffusion", Image and Graphics, 2004. Proceedings. Third International Conference on18-20 Dec. 2004 Page(s):274 - 277

[16] L. Hong, Y. Wan and A. K. Jain, "Fingerprint Image Enhancement: Algorithm and Performance Evaluation", IEEE Transactions on Pattern Analysis and Machine Intelligence, vol. 20, no. 8, August 1998.

[17] R. C. Gonzalez and R. E. Woods, Digital Image Processing, Pearson Education (Singapore) 2003.

[18] J. Kybic and M. Unser, "Fast Parametric Elastic Image Registration", IEEE Transactions on Image Processing, vol. 12, no. 11, November 2003.

[19] L. Brown, “A survey of image registration techniques," ACM Computer Survey, vol. 24, no. 4, pp. 326-376, Dec. 1992.

[20] T. K. Shih and R. C. Chang, "Digital Inpainting - Survey and Multilayer Image Inpainting Algorithms (Keynote Paper)," in Proceedings of the 3rd International Conference on Information Technology \& Applications (ICITA 2005), Sydney, Australia, July 4 - 7, 2005.

[21] A. Criminisi, P. Pérez, and K. Toyama, "Region Filling and Object Removal by Exemplar-Based Image Inpainting”, IEEE Transactions on Image Processing, vol.13, no. 9, September 2004.

[22] X. Li and M. Orchard "Novel Sequential Error Concealment Using Orientation Adaptive Interpolation", IEEE Trans. on Circuits and System for Video Tech., vol. 12, no. 10, pp. 857-864, Oct. 2002.

[23] M M. Oliveira, B. Bowen, R. McKenna and Y. S. Chang, "Fast Digital Image Inpainting", Appeared in the Proceedings of the International Conference on Visualization, Imaging and Image Processing (VIIP 2001), Marbella, Spain. September 3-5, 2001.

[24] H. Yamauchi, J. Haber, and Hans. P. Seidel, "Image Restoration using Multiresolution Texture and Image Inpainting", Proceedings of the Computer Graphics International (CGI'03) 1530-1052, 2003 IEEE. 
[25] J. Gir, S. Peng and X. Wang, "Digital Image Inpainting using Monte Carlo Method”, 2004 International Conference on Image Processing (ICIP).

[26] Tabassi, E., Wilson, C., Watson, C.: Fingerprint image quality (NIST Research Report NISTIR 7151, August 2004).

[27] M. Y. Yao, S. Pantanki, N. Haas, N. Ratha, and R. Bolle, "Quantifying Quality: A case study in fingerprints", Proc. of IEEE Conference on AutoID, Tarrytown, NY, March 2002.

[28] E. Lim; X. Jiang; and W. Yau, "Fingerprint quality and validity analysis", Image Processing. 2002. Proceedings. 2002 International Conference on Volume 1, 22-25 Sept. 2002 Page(s): I-469 - I-472.

[29] J. Fierrez-Aguilar, Yi Chen, J. Ortega-Garcia and A. K. Jain, "Incorporating Image Quality in Multi-algorithm fingerprint Verification", Proc. of International Conference on Biometrics (ICB), pp. 213-220, Hong Kong, Jan 2006.

[30] T. Kamei and M. Mizoguchi, Image Filter Design for Fingerprint Enhancement, Proc. ZSCV'95, Coral Gables, FL, pp. 109-1 14, 1995.

[31] A.M. Bazen, S. Gerez, "Fingerprint matching by thin-plate spline modelling of elastic deformations", Pattern Recognition 36 (8) (2003) 1859-1867.

[32] A.M. Bazen, S. Gerez, "Elastic Minutiae Matching by Means of Thin-Plate Spline Models", 16th International Conference on Pattern Recognition (ICPR'02) - Volume 2 p. 20985.

[33] A.M. Bazen, S. Gerez, "Systematic Methods for the Computation of the Directional Fields and Singular Points of Fingerprints", IEEE Transactions on Pattern Analysis and Machine Intelligence, Vol. 24, no. 7, July 2002.

[34] N. Ratha, S. Chen and A.K. Jain, "Adaptive Flow Orientation Based Feature Extraction in Fingerprint Images", Pattern Recognition, Vol. 28, pp. 1657-1672, November 1995.

[35] Z. Shi, Y. Wang, J. Qi, and Ke Xu, “A New Segmentation Algorithm for Low Quality Fingerprint Image", Image and Graphics, 2004. Proceedings. Third International Conference on 18-20 Dec. 2004 Page(s):314 - 317.

[36] S. Park, M.J.T. Smith, and J. J. Lee, "Fingerprint enhancement based on the directional filter bank", Image Processing, 2000. Proceedings. 2000 International Conference on Volume 3, 10-13 Sept. 2000 Page(s):793 - 796. 
[37] S. Wang and Y. Wang, "Fingerprint Enhancement in the Singular Point Area", IEEE Signal Processing Letters, Vol. 11, no. 1, January 2004.

[38] S. Ghosal, N. K. Ratha, R. Udupa and S. Pankanti, "Hierarchical Partitioned Least Square Filter-Bank for Fingerprint Enhancement", Pattern Recognition, 2000. Proceedings.15th International Conference on Volume 3, 3-7 Sept. 2000 Page(s):334 - 337 .

[39] V. Areekul, U. Watchareeruetai, K. Suppasriwasuseth and S. Tantaratana, "Separable Gabor Filter Realization for Fast Fingerprint Enhancement", Image Processing, 2005. ICIP 2005. IEEE International Conference on Volume 3, 1114 Sept. 2005 Page(s):253 - 256.

[40] Yuan-Ning Liu, Zhi-Hui Li, "Research on fingerprint enhancement and classification algorithm", Computer and Information Technology, 2004. CIT '04. The Fourth International Conference on 14-16 Sept. 2004 Page(s): 199 - 204.

[41] T. Kamei, M. Mizoguchi, "Image filter design for fingerprint enhancement", Computer Vision, 1995. Proceedings, International Symposium on 21-23 Nov. 1995 Page(s): 109 - 114.

[42] B. Rusyn, I. Prudyus, V. Ostap, "Fingerprint image enhancement algorithm", CAD Systems in Microelectronics, 2001. CADSM 2001. Proceedings of the 6th International Conference. The Experience of Designing and Application of 12-17 Feb. 2001 Page(s):193 - 194.

[43] J. Wang, H. Shen, and R. Ding, "A novel fingerprint image enhancement algorithm using phase preserving", Communications and Information Technology, 2005. ISCIT 2005. IEEE International Symposium on Volume 2, 12-14 Oct. 2005 Page(s):1472 - 1475.

[44] N. Ikeda, M. Nakanishi, K. Fujii, T. Hatano, S. Shigematsu, T. Adachi, Y. Okazaki, and H. Kyuragi, "Fingerprint image enhancement by pixel-parallel processing", Pattern Recognition, 2002. Proceedings. 16th International Conference on Volume 3, 11-15 Aug. 2002 Page(s):752 - 755 vol.3.

[45] P-E. Danielsson and Ye Qun-Zhong, "Rotation-invariant operators applied to enhancement of fingerprints", 9th International Conference on Pattern Recognition, 1988., 14-17 Nov. 1988 Page(s):329 - 333 vol.1. 
[46] T. K. Shih., R-C. Chang, L-C. Lu, W-C. Ko and C-C. Wang, "Adaptive Digital Image Inpainting", Proceedings of the 18th International Conference on Advanced Information Networking and Application (AINA'04).

[47] M. Bertalmio, L. Vese, G. Sapiro, and S. Osher, "Simultaneous Structure and Texture Image Inpainting", Proceedings of the 2003 IEEE Computer Society Conference on Computer Vision and Pattern Recognition (CVPR'03).

[48] A. Rares, Marcel J. T. Reinders, and Jan Biemond, "Edge-Based Image Restoration", IEEE Transactions on Image Processing, Vol. 14, no. 10, October 2005.

[49] T.K. Shih, Liang-Chen Lu, Y-H. Wang, and R-C. Chang, "Multi-resolution image inpainting", Multimedia and Expo, 2003. ICME '03. Proceedings. 2003 International Conference on Volume 1, 6-9 July 2003 Page(s):I - 485-8.

[50] Alexei A. Efros and Thomas K. Leung, "Texture Synthesis by Non-parametric Sampling", IEEE International Conference on Computer Vision, Corfu, Greece, September 1999.

[51] A. Papoulis, Probability, Random Variables and Stochastic Process. New York: McGraw-Hill, 1984.

[52] N. Jayant and P. Noll, Digital Coding of Waveforms: Principles and Applications to Speech and Video. Englewood Cliffs, NJ: Prentice-Hall, 1984.

[53] X. Li and M. Orchard, "Edge directed prediction for lossless compression of natural images," IEEE Trans. Image Processing, vol. 10, pp. 813-817, Jun. 2001.

[54] B.K.P. Horn and B.G. Schunck, "Determining Optical Flow", Artificial Intelligence, 17, 1981, pp185-204.

[55] J. L. Barron, N. A. Thacker, "Tutorial: Computing 2D and 3D Optical Flow," Imaging Science and Biomedical Engineering Division, Medical School, University of Manchester, Jan. 2005.

[56] B.D. Lucas and T. Kanade, "An Iterative Image Registration Technique with an Application to Stereo Vision”, DARPA Image Understanding Workshop, 1981, pp121-130 (see also IJCAI'81, pp674-679).

[57] J. L. Barron. Experience with 3D optical flow on gated MRI cardiac datasets. In 1st Canadian Conference on Computer and Robot Vision, pages 370-377, 2004. 
[58] A. K. Jain, S. Prabhakar and S. Pankanti, "Filterbank-based fingerprint matching", IEEE Transactions on Image Processing, vol. 9, no. 5, May 2000.

[59] Mansfield, A. J., \& Wayman, J. L. (August 2002),” Best Practices in Testing and Reporting Performances of Biometric Devices", Version 2.01 (NPL Report CMSC 14/02). UK:National Physical Laboratory. 